\title{
MASTER
}

[ICRI-52751

\section{Extreme ultraviolet diagnosis of a neutral-beam-heated mirror machine}

\author{
R. Paul Drake
}

July 1980

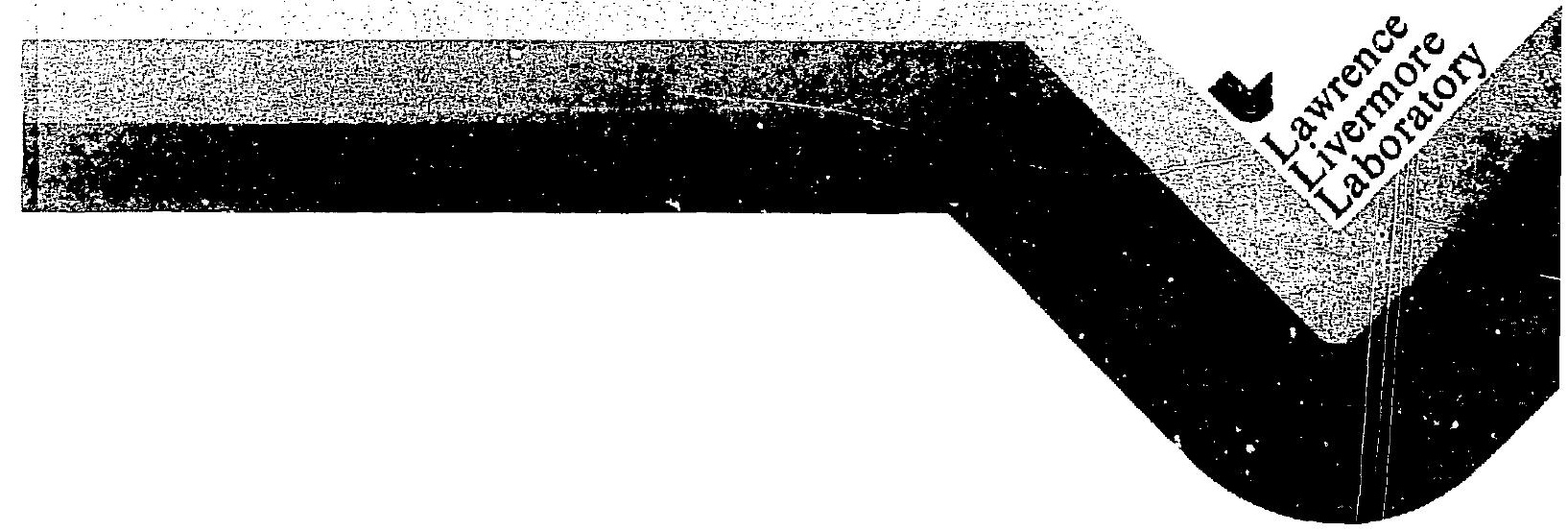




\title{
Extreme ultraviolet diagnosis of a neutral-beam-heated mirror machine
}

\author{
R. Paul Drake
}

July 1980

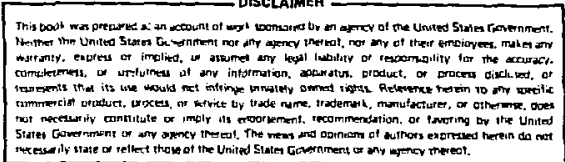

\section{LAWRENCE LIVERMORE LABORATORY University of California $\bullet$ Livermore, California $\bullet 94550$}


The Magnetic Fusion Energy Division of the Lawrence Livermore National Laboratory provided the opportunity for cooperative research, and contributed to its success. The 2 XIIB research physicists all encouraged me, and provided many helpful, stimulating, and informative discussions. R. S. Hornady initiated the entire project. His help was crucial in beginning the experiment and obtaining the data. T. C. Simonen supported my efforts and helped me learn, giving much of his own time. Others who provided feedback, assistance, and constructive criticism include D. E. Baldwin, J. F. Clauser, W. C. Condit, D. L. Correli, W. F. Cummins, J. H. Foote, A. H. Futch, R. K. Goodman, D. P. Grubb, A. L. Hunt, B. G. Logan, G. M. Melin, W. F. Nexsen, B. W. Stallard, and W. C. Turner. The technical staff at Livermore contributed, helping to find and install needed equipment and electronics. Finally, the secretarial staff at Livermore and Johns Hopkins helped me willingly and cheerfully. Without them, even if I didn't strangle in red trpe, my project would have been completed less quickly and less we11. Martelle Mays typed the manuscript, and did an impressive job. 


\section{ABSTRACT}

E:.treme ultraviolet emissions from the LLL 2XIIB fusion research experiment have been studied. (2XIIB was a magnetic-mirror-plasmaconfinement device; beams of high-energy ( $20 \mathrm{keV}$ ) neutral deuterium created a high-density, high-temperature plasma.) A normal-incidence concave-grating monochromator, equipped with a windowless photomultiplier tube, was used to measure emissions in the spectral region from 400 Angstrom to 1600 A. Emissions of oxygen, titanium, carbon, nitrogen, and deuterium were identified; the oxygen brightnesses at times exceeded $10^{18} \mathrm{ph}^{-1}-\mathrm{cm}^{-2}-\mathrm{sr}^{-1}$. A survey of the emission characteristics found the oxygen concentration was $3 \%$, the other impurities had concentrations near $0.4 \%$. The radiated power loss was about $5 \%$ of the deposited neutral beam power.

The discovery of a $3 \%$ oxygen concentration was unexpected, because it had been supposed that the electric field of the plasma would eject the im purities. An extensive study of oxygen emissions showed that the oxygen was injerted by the neutral beams and had a substantial mean energy (10 kev). In consequence, the oxygen was confined by the magnetic fields in 2 XIIB and was not affected by the electric field of the plasma. The distribution of the oxygen ions anong the possible ionization states was found to be determined by a balance of electron impact ionization and charge-exchange between the oxygen ions and deuterium atoms. The detailed effects of oxygen on the plasma power balance were also evaluated.

The effect of the electric field of the plasma on low-energy impurities was studied by injecting neon into 2 XIIB. In the first experiment, neon plasma was injected along the magnetic field lines. Most of the injected 
I. Introduction 1

II. The 2XIIB Confinement Device 5

A. Introduction 5

B. Description of 2XIIB 5

C. The Physical Principles Which Govern Mirror Machines 7

D. Recent 2XIIB Operation 13

III. Spectroscopic Instrumentation and Theory 15

$\begin{array}{ll}\text { A. Introduction } & 15\end{array}$

B. The Spectrometer 15

C. Mounting of the $0.4-m$ Instrument on $2 X I I B \quad 18$

$\begin{array}{ll}\text { D. Atomic Processes } & 18\end{array}$

E. Measurement of Brightnesses, Densities,
and Concentrations

IV. Impurity Emissions, Concentration, and Power Loss 29

A. Introduction 29

B. EUV Emissions From 2XIIB 29

C. Concentrations and Power Loss 35

V. Results of a Detailed Study of Oxygen Emissions 37

A. Introduction $\quad 37$

B. Evidence for Neutra1-Beam-Injected Oxygen 37

c. The Population of Oxygen Ionization States 44

D. The Effects of Oxygen on the $2 X I I B$ Power Balance $\quad 49$

VI. The Plasma Potential and Neon Transport 55

A. Introduction

B. Summary of Neon Streaming Plasma Penetration 55

C. Description and Summary of Radial Puffing 56 
DETAILED CONTENTS OF TEXT AND APPENDICES (continued)

Page

D. Brightness Versus Loss Mechanism 58

E. Neon Fluxes and Neon Confinement 61

$\begin{array}{lr}\text { VII. Conclusions } & 67\end{array}$

A. Summary of These Results 67

B. A General Discussion of Impurities

C. EUV Diagnosis of the Tandem Mirror

Experiment (TMX) 72

D. EUV Diagnosis of the Mirror Fusion

Test Facility (MFTF)

Appendices

A. Ion Impact Collision Processes 79

$\begin{array}{ll}\text { Al. Introduction } & 79\end{array}$

A2. Calculation Method 80

A3. Ionization 81

A4. Deuteron Impact Excitation 84

B. The Neutral Density in 2XIIB 87

$\begin{array}{ll}\text { B1. Introduction } & 87\end{array}$

B2. Calculation of Neutral Densities 87

B3. Scaling of the Neutral Density 90

C. A Survey of Impurities in 2XIIB 93

Cl. Introduction 93

c2. Measurements of 2XIIB Impurity Emissions 93

C3. Impurity Concentrations in 2XIIB 105

C4. Radiated Power Loss 111

$\begin{array}{ll}\text { D. Spatial Scans of 2XIIB } & 117\end{array}$

E. A Model of Beam Injection of 0xygen 125 
DETAILED CONTENTS OF TEXT AND APPENDICES (continued)

$\underline{\text { Page }}$

F. Possible Sources of Carbon, Titanium, and Nitrogen

F1. Introduction

F2. Carbon Studies

F3. Titanium and Nitrogen

138

G. Details of the Neon Injection Experiments

Gl. Introduction

G2. Injection of Neon Streaming Plasma

G3. Radial Puffing

H. EUV Data Pertaining to the Plasma Boundary

Hl. Introduction

H2. Imp1ications of Impurity Spatial Data

160

H3. Implications of the Lyman Alpha Data

161 
1 Schematic of $2 X I I B$ and selected diagnostics, including the EUV monochromator system.

2 Properties of a mirror machine. (a) The magnetic field (B), plasma density $(n)$, and potential ( $\phi$ ) along the magnetic axis (z) are shown. (b) The ambipolar hole is the extension of the loss cone due to the plasma potential.

3 Schematic of the $0.4-m$ monochromator.

4 The magnetic shielding of the EUV detector. Note that the open ends of the inner and outer shields overlap, reducing field penetration through the ends.

5 A typical 0 V $630 \AA$ time development. The electron line density (dashed line) and the beam and stream durations are also shown. Some plasma parameters are given.

6 Typical emissions of the observed oxygen ions, from identical shots on 24 0ctober 1977.

7 (a) 0 V 630- $\AA$ brightness and electron line density profiles are shown. (b) $0 \mathrm{~V}$ density profiles given by Abel inversion and two models are shown; see text for details.

8 The 0 II brightness was observed to scale as the neutra1-beam current to the 1.6 power, and it was successfully modeled. C III emissions scale differently.

9 The 0 II $539 \&$ brightness and the electron line density profiles are shown above. Below, the variation of density profiles allowed by the data is indicated.

10 Observed and predicted population distributions of oxygen among the observed ionization states. The observed distributions (solid lines) vary considerably as plasma conditions change. The predictions agree adequately with the data. The use of ionization rate coefficients equal to 0.6 times the tabulated values (Lotz [24]) gives better agreement than the full values do.

11 The observed neon fluxes show a sharp decline as charge state increases, for two different gas-valve-supply pressures.

12 The results of a simple model suggest that the observed neon was confined for much less than $100 \mu \mathrm{s}$.

B1 The transmission of the neutral beams through plasmas of various line densities is shown. A Gaussian spatial density profile was assumed. 
cl Spectral scans of titanium emissions show the triangular $2 \AA$ FWHM profile of the instrument transmission.

C2 A spectral scan of C III $977 \AA$ emissions, showing that the line becomes broad during the shot.

C3 A spectral scan of the 0 VI $1034 \AA$ doublet. Doppler broadening and the expected 2:1 brightness ratio were observed.

C4 Spectral scans of $0 \mathrm{~V}$ emissions also showed substantial Doppler broadening.

C5 Spatial profiles of C III $977 \AA$ A brightness and electron line density are shown above. A density profile obtained by Abel inversion and the density given by the constant density model (dashed line) are shown below.

Dl Spatial scan of N III $685 \AA$.

D2 Spatial scans of Ti IV $779 \AA$.

D3 Spatial scans of $0 \mathrm{~V} 630 \AA$.

D4 Spatial scans of 0 II $539 \AA$.

D5 Spatial scans of 0 II $539 \AA$.

D6 Spatial scan of C III $977 \AA$.

F1 C III $977 \AA$ emissions declined during the first few shots after $2 X I I B$ was evacuated.

Gl Schematic of the neon plasma injection experiment. 142

G2 P1asma characteristics during neon plasma injection. 143

G3 The time development of emissions from Ne II through Ne $\mathrm{V}$ during plasma injection shows the successive appearance of emissions from more highly ionized states.

G4 Spatial scans during plasma injection show the broad distribution of the neon.

65 During the neon plasma injection experiment, the ion end loss current and energy distribution were determined using a gridded analyzer. The westward current is normalized to the midplane magnetic field. The increase in measured current from zero to 140 volts repeller voltage is unphysical, and is indicative of the scatter in the data. 
G6 When neon was used, instead of deuterium, to stabilize the plasma, more intense fluctuations were observed.

G7 Schematic of the neon gas puffing experiment.

G8 Spatial data obtained during neon gas puffing showed asymmetric brightness profiles.

G9 The Ne IV $544 \AA$ time development was observed as the gas pressure in the gas valve supply line was increased. The signal prior to $3.5 \mathrm{~ms}$ is background.

G10 The Ne V $571 \AA$ emissions increased as the gas-valve-supply pressure was raised, which increased the neon flux striking the plasma.

G11 The Ne III $490 \AA$ brighiness increased when the neon gas flux was raised by increasing the pressure in the gas-valvesupply line.

G12 The neon flux striking the plasma was increased by raising the gas valve supply pressure ( 0 to 400 Torr were used). Plasma diamagnetism and electron line density are shown.

G13 The energy distributions and end loss currents were affected little until the plasma was destroyed.

Hl The Lyman alpha time development, electron line density, and bean and stream durations are shown. Wide slits were used to measure the entire line. 
$1 \quad$ Impurity Time Scales $\quad 20$

2 Formulas for Impurity Lifetimes 21

3 Impurity Energies From Doppler Broadening 35

4 Summary of Impurity Concentrations 36

5 Oxygen Population Distributions $\quad 47$

6 The Effects of Oxygen on the 2 XIIB Power Balance 50

7 Brightnesses and Fluxes During Neon Gas Puffing 59

8 Mirror Reactor Power Densities 70

9 Useful EUV Instrumentation for TMX 75

A1 Ion Impact Ionization (Outer She11) 82

A2 Double Ionization by Ion Impact 83

A3 Deuteron Impact Excitation $\quad 85$

B1 Neutral Densities in 2XIIB 90

Cl Observed Ions and Transitions 94

C2 Emission Lines Unresolved From Background 96

C3 Some Measured Brightnesses and Plasma Parameters 97

C4 Brightnesses of $0 \vee 630$ A on Various Days 100

c5 Detailed Impurity Concentration Results 108

$\begin{array}{ll}\text { G6 Streaming Plasma Observations } & 110\end{array}$

c7 Light Impurity Radiated Power Loss 115

F1 Carbon Cleanup Data 133

G1 Plasma Parameters During Neon Plasma Injection 144

G2 Neon Densities During Plasma Injection $\quad 148$

H1 Low-Energy Deuterium Fluxes $\quad 164$ 


\section{INTRODUCTINN}

Controlled thermonuclear fusion reactions used to prodtice electric power could we11 become an important and common part of our !'ves: Fusion reactors will heat deuterium, extracted from seawater, to such high temperatures that deuteriu.! nuclei overcome their natural repulsion and fuse, creating helium and releasing energy, But absorbing the capital costs of fusion power plants is not yet a concern of economists. In fact, engineers have just begun to design workable fusion reactors. Today, physicists are still learning how to produce the physical conditions necessary to achieve fusion power.

These conditicrs include high density to achieve a high reaction rate, good energy confinement to attain efficient burning, and the high temperature mentioned above. Two basic techniques are now being tried. The alternatives are heating the fuel very rapidly and evenly so it produces power before it can blow itself apart (inertial confinement), or confining and heating the fuel within a strong magnetic field. At present, the most successful mag netic confinement device is the tokamak, which traps the ionized fuel (a plasma) within a doughnut-shaped magnetic field. However, tokamak reactors would be inconveniently large and complex. An altemative is the magnetic mirror machine. A particle moving into an increasing magnetic field will slow down and turn around (a consequence of the Lorentz force and conservation of angular momentum), unless its velocity is completely parallel to the fiald. A mixror machine traps particles between two regions of increasing magnetic fielu, known as magnetic mirrors. Present mirror confined plasmas can contain substantial energy, but require a high power input. Incressing the energy confinement time, and hence decreasing the power 
required to sustain a high-energy plasma, is the central goal of current mirror research.

To eventually achieve fusion power, it is necessary to study present devices to learn how to design larger and more powerful machines. The field of plasma diagnostics does this, and plasma spectroscopy, one part of plasma diagnostics, studies plasmas by analyzing the electromagnetic radiation they emit. Extreme uitraviolet (EUV) spectroscopy, which studies radiation with wavelengths between 50 and $2000 \AA$, is a useful tool because it is comparatively easy to interpret. Spectral-1ine emissions from neutral deuterium and incompletely ionized impurities can be measured. By this method, impurities and radiation power loss can be studied, as well as certain more subtle plasma characteristics. This report describes one of the very few EUV diagnoses of a major mirror machine, probably the most extensive to date.

Some background regarding the origin and initiation of this study is worth relating. Instabilities caused early mirror machines to have very poor energy confinement [1]. It is only recently (1975) that neutral-beam heating and increased ability to control instabilities enabled the 2XIIB device to achieve substantial improverients in energy confinement and the highest ion temperatures ever obtained in a major fusion research device [2]. Until then, circumventing the instabilities had been so important that few impurity studies were performed. The other uses of EUV spectroscopy were not thought to justify its cost.

In addition, there were sound physical arguments suggesting that impurities should be unimportant in mirror machines. The high charge of impurity ions, relative to deuterius, causes them to be more quickly scattered by Coulomb collisions, and to be strongly repelled by the electric 
field of the plasma. This means they can escape the magnetic mirror more readily [ 3$]$.

In view of these arguments, the discovery of an oxygen concentration of a few percent in 2XIIB, which occurred early in the study reported here, was quite perplexing. A major result of this study is that the oxygen discovered in 2 XIIB is now understood. It is injected by the neutral beams and is confined because it has a large velocity perpendicular to the magnetic field. In addition, neon injection experiments have provided evidence supporting the second argument given above: low-energy impurities are quickly ejected. In contrast, the transport and heating of the observed carbon and titanium impurities, which are not beam-injected, remain to ie understood.

This report is designed to acquaint the reader with the necessary background before presenting the data and analysis. The 2XIIB device and the physical principles of mirror machines are discussed in Section II, with emphasis on those aspects that affect the impurities in the plasma. The spectrometer, the atomic processes affecting impurities, and the analys is of the EUV data are described in Section III. 2XIIB differs considerably from the tokamaks previously stidied with EUV spectroscopy. A general approach was taken, to ensure the models chosen applied to 2 XIIB. The results of the general survey of impurities in $2 X I I B$ are summarized in section IV. The EUV emissions, concentrations, and radiated power loss are described. Appendix C presents the results of the survey in more detail.

After the initial jis covery of an oxygen concentration of a few percent, oxygen emissions were studied at length. Section V explains the consequent understanding of the source and confinement of oxygen. The degree of ionization of the oxygen impurities, which is stror zly affected by charge- 
exchange processes, is discussed. The effect of oxygen on the plasma power balance is also considered.

Neon was injected into 2 XIIB in the attempt to observe the effects of the plasina potential on low-energy impurities. Section VI presents the results of these experiments, which included the injection of neon ions along magnetic field lines and the puffing of neon gas toward the plasma. The details of the neon injection experiments, and some results from them pertaining to plasma stability, are found in Appendix G. The conclusions of this study are sumarized in Sectinn VII, which then considers some of its implications, both for impurities in future mirror machines and for the possible uses of EUV spectroscopy in mirror research.

The appendixes consider topics that are significant, but too detailed to fit smoothly into the main text. Because 2 XIIB has a very high mean ion energy, ion-impact collision processes should be considered; and this is done in Appendix A. Appendix B evaluates the neutral density, which is needed to calculate the rates of charge-exchange processes. Appendix $C$ presents the survey of impurity emissions in detail; and Appendix D contains additional spatial data. Appendix E presents a model of the injection of neutral-beam oxygen, which is used in Section $\mathrm{V}$ to evaluate some oxygen data. Although the sources of carbon, titanium, and nitrogen are not known, some evidence exists; this is sumarized in Appendix F. Appendix G sumarizes the neon experiments, as mentioned above; and Appendix $H$ evaluates the implications of the EUV data regarding the plasma boundary. 


\section{THE 2XIIB CONFINEMENT DEVICE}

\section{A. Introduction}

The 2XIIB device and its functioning are described in this section. Special attention is paid to those aspects which affect EUV emissions. A physical description of 2 XIIB forms the next section. It is followed by a sumary of the physical principles and processes which govern the behavior of the plasma and its impurities. The final section describes the recent plasma physics experiments performed using 2 XIIB; it al so directs the reader to more detailed discussions. Section III considers the spectrometer used to measure EUV emissions, and the interpretation of the spectroscopic data.

\section{B. Description of 2 XIIB}

The 2XIIB magnetic mirror confinement device produces dense, highenergy plasmas. This device has been described elsewhere [2]; but, because the arguments below are based on its nature, this section describes its components and functioning with reference to Fig. 1 .

The 2XIIB magnetic field is a combination of a quadrupole field and a guide field. The guide field, which is parallel to the arrow marked "stream" in Fig. 1, allows plasma to penetrate and leave the central part of the machine along field lines. The quadrupole field is product by a yin-yang coil set, which creates a "minimum-B" magnetic well (e.g., the magnetic field has its minimum value at machine center, and increases in every direction). This produces magnetic mirrors in the direction of the guide field. While the magnetic field (and hence the plasma) has a cylindrical cross section near the midplane, it becomes elliptical as it passes through the mirrors and into the end tanks. The resulting "end fans" are referred to below, but only the central plasma was studied during this experiment. 
JHU monochromator

Thompson scattering

Ruby laser

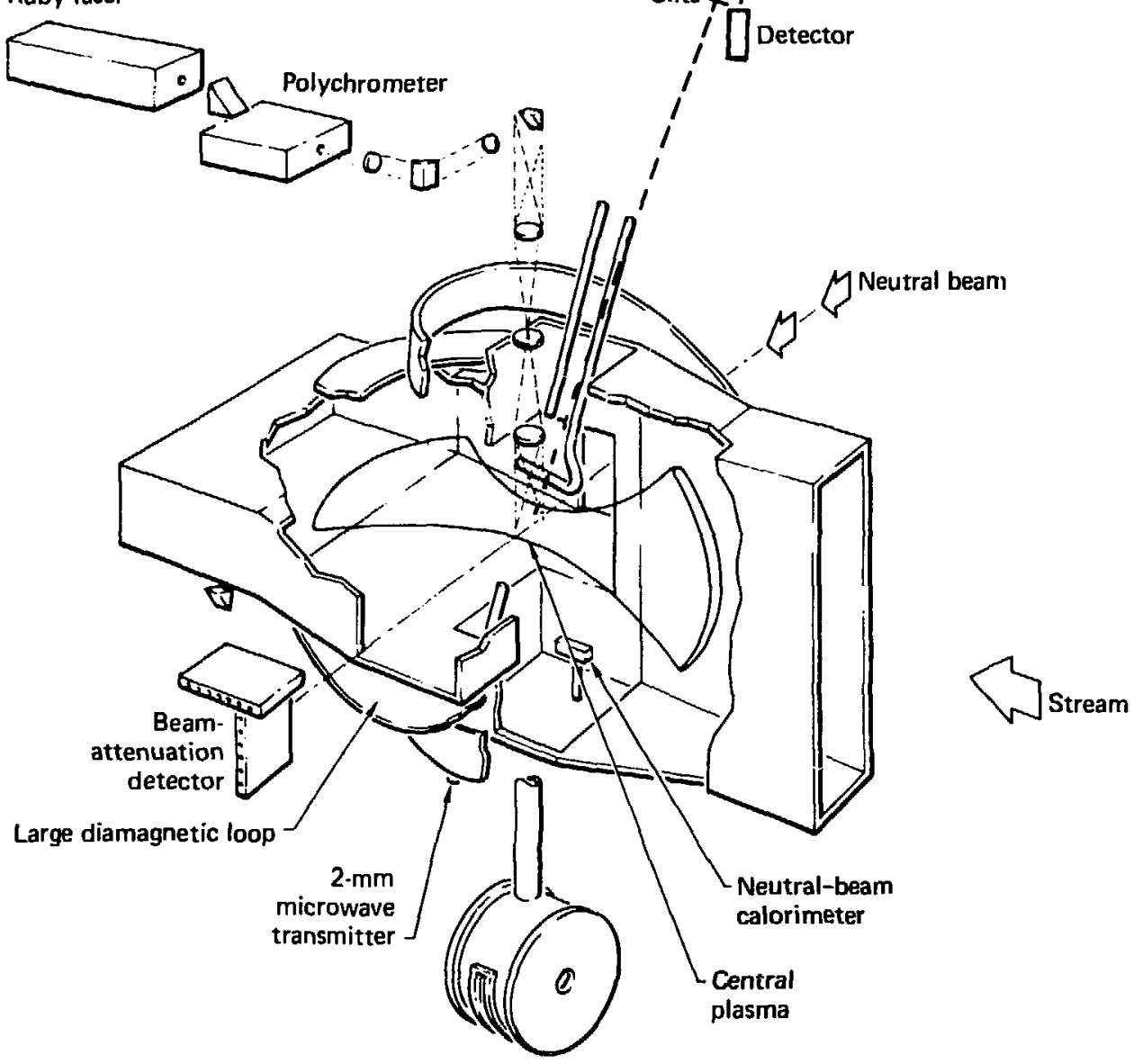

15 channel charge-

exchange analyzer

Figure 1. Schematic of 2 XIIB and selected diagnostics, including the EUV monochromator system. 
The sources of plasma and energy will now be described. Plasma is injected through the mirrors along field lines. (This is labeled "stream" in the figure.) High-energy beams of deuterium strike the streaming plasma. The neutral beams are absorbed by means of charge-exchange and ionization; and a high-energy, high-density, high-beta plasma is created $\left(E_{i} \sim 12 \mathrm{keV}\right.$, $\left.T_{e} \sim 100 \mathrm{eV}, n_{e} \sim 5 \times 10^{13}, \beta \sim 0.5\right)$. Beta $(\beta)$ is the ratio of particle pressure to vacuum magnetic field pressure, and this device has achieved betas as high as two.

The entire 2XIIB device is evacuated. The 57,000 -litre vacuum system is kept near $10^{-8}$ torr, although the pressure rises during a shot. The vacuum is maintained by diffusion pumping and titanium gettering; liquidnitrogen-cooled surfaces are sometimes also used. This results in a low gas load on the plasma, and requires the use of diagnostics with good vacum properties.

\section{The Physical Principles Which Govern Mirror Machines}

The behavior of mirror confinement devices is described in this section. The important physical principles and processes are summarized, with emphas is on those which affect impurities. This section is not intended to be a thorough presentation of the subject. Rather, it should give the reader a basic familiarity with the working of mirror machines, sufficient to understand and evaluate the argments presented below. BaIdwin [4] provides a more thorough review of some of these principles.

\section{Mirror Confi nement}

The simplest mirror machine is a pair of Helmboltz coils. The magnetic field, which is strongest near the coils, traps particles between them. Some 
of the particles escape because their velocity vectors are not sufficiently perpendicular to the magnetic field. These particles are said to be in the "loss cone" in velocity space. The remaining particles would appear to be confined until they scatter into the loss cone.

While no single magnetically trapped particle can escape the simple mirror on its own, the piasma, acting as a magnetic fluid, can escape by means of a magnetohydrodynamic instability. In hydrodynamics, it is we11known that when a light fluid supports a heavy fluid against the force of gravity, the equilibrium is unstable because small perturbations tend to grow. In a simple mirror, the centrifugal force is outward as the plasma moves along the field lines. In consequence, the vacuum (a light fluid) is supporting the plasma (a heavy fluid) against the centrifugal force (gravity), and small perturbations grow exponentially. To prevent this instability, the centrifugal force must be reversed, which is accomplished by changing the shape of the field lines. The quadrupole field created in $2 \times$ IIB is one example of a stable magnetic configuration. Mathematical discussions of the simple details of mirror confinement can be found in basic texts on plasma physics.

The plasma confined within a properly shaped magnetic field soon develops a positive potential. The electrons in the plasma collide and scatter many times faster than the ions do. As a result, many electrons enter the $108 \mathrm{~s}$ cone and try to leave the plasma, more quickly than the ions. This creates electric field which confines the electrons and ejects some low energy ions. A steady state is reached when the electron and ion loss rates are the same. The plasma potential in 2 XIIB, which is about $3 \mathrm{~T}_{\mathrm{e}} / \mathrm{e}$ [5], confines all but the most energetic electrons. Near the machine center, the potential variation along field lines is given by the Boltzmann relation, 
$n_{e}(z)=n_{e}(0) \exp \left[-e_{\phi}(z) / k T_{e}\right]$. Near the magnetic mirrors this is not true, because of electron temperature gradients [6].

Figure 2 is a schematic that shows most of the features described above. The plasma density $n$ is confined within the magnetic mirrors, and the plasma potential $\phi$ is established accordingly. Velocity space is divided into a confined region and an unconfined region. The latter is enlarged due to the plasma potential. The added unconfined region is known as the "ambipolar hole." When an ion falls into it (by losing energy), the ion escapes from the plasma.

(a)

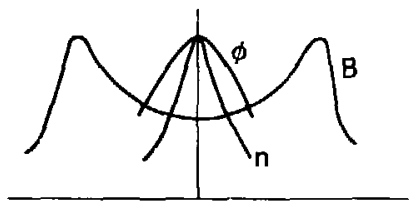

Z. (magnetic axis)

Configuration space

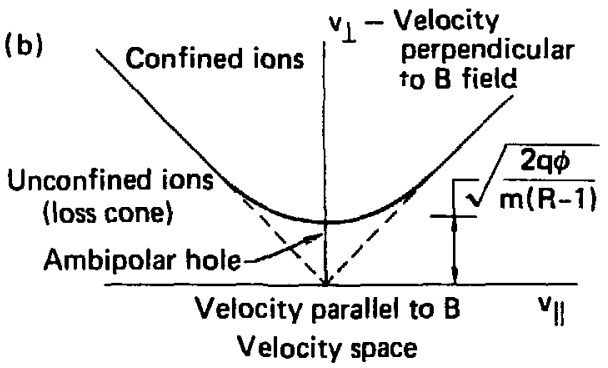

Figure 2. Properties of a mirror machine. (a) The magnetic field ( $B$ ), plasma density $(n)$, and potential $(\phi)$ along the magnetic axis (z) are shown. (b) The ambipolar hole is the extension of the loss cone due to the plasma potential. 
The existence of the plasma potential (or the "ambipolar hole" in velocity space) is the basis of one argument that impurities should be unimportant in a mirror machine. It is very difficult for a multiply ionized impurity to remain confined, because its ambipolar hole is many times larger than the hole seen by a deuterium ion. Most impurity sources produce lowenergy impurities. When these impurities enter the plasma, they wil1 in general be ionized by the hot electrons and ejected by the plasma potential. As is discussed below, the EUV data supports this argument, but it does not apply to neutral-beam-injected impurities.

Another argument that impurities should be poorly confined in a mirror device is based on their enhanced ion-ion scattering [3]. Impurities scatter into the loss cone more quickly than deuterium ions do, because their high charge makes Coulomb collisions more effective in changing their momentum. (This argument has little application to $2 X I I B$, in which the ion confinement is not dominated by ion-ion scattering.)

\section{Neutral-Beam Heating}

High-energy neutral beams can easily heat mirror-confined plasmas (once such beams are available). Neutral-beam heating has many effects on the plasma and the processes that occur within it. Some of these effects will now be explored.

Most of the particles injected by the neutral beams leave the plasma as neutral charge-exchange products and strike the machine walls. Only a small fraction of the injected beam current increases the plasma density (this will not be true in the larger mirror machines of the future). The large neutral current striking the walls (a few hundred amps) can produce cold gas reflux, 
secondary electrons, and impurities. As discussed in Appendix F, this is a possible source of the carbon observed in $2 X I I B$.

It is important that the neutral density in $2 \times 1 I B$ be high; this affects atomic processes within the plasma. The high neutral density results from the small size of the plasma, which is not optically thick to $20-\mathrm{kV}$ beam penetration. The neutral beams are partially transmitted through the plasma; in consequence the density of high-energy neutrals is 1 arge $\left(n_{D} \sim 10^{-3} n_{e}\right.$ ). These atoms of ten transfer their electrons to highly ionized impurities (charge-exchange), with effects discussed in sections III-D and V-C. In addition, the frequent charge-exchange of these neutral atoms with plasma ions keeps the mean ion energy in 2XIIB close to the injection energy.

Instabilities and the Electron Temperature

As noted in Section I, the understanding and control of instabilities has been crucial to the success of mirror machines. In $2 X I I B$, the driftcyclotron, loss-cone instability dominates the plasma fluctuations [7]. It is produced by the combiried existence of the ambipolar hole, shown in Fig. 2, and the plasma density gradient. The ambipolar hole can be partially filled by the continuous injection of streaming plasma, which is necessary to keep the 2XIIB plasma marginally stable [8]. Unfortunately, present sources of streaming plasma al so cool the electrons, reducing the electron temperature below its optimum value. During this study, the 2XIIB plasma was maintained using a neon streaming plasma rather than a deuterium one. This is somewhat contrary to expectations and is discussed in Appendix G.

The electron temperature in a wirror machine is generally much lower than the ion temperature. The neutral beams inject hot ions and cold electrons into the plasma (the heavier nuclei keep most of the energy when the 
neutral atoms are broken up). The ions then heat the electrons by means of many minor Coulomb collisions; this friction-like process is known as Spitzer drag [4]. Because of iom-ion scattering and the presence of an ambipolar potential which is several times $T_{e}$, the ions can never be confined long enough to equilibrate with the electrons. A mathematical treatment of the electron energy balance [4] shows that the classical limit on the mean electron energy in a large, stable mirror machine is one-tenth the ion energy (if there is no supplementary electron heating).

In 2 XIIB the mean ion energy is about 100 times the electron temperature. As was described above, the mean, ion energy is kept bigh by frequent charge-exchange and the electrons a re cooled by the streaming plasma. In consequence, the ion temperature is high and the electron temperature is low compared to an "ideal" mirror device.

Because the ion energies are large in comparison with the electron energies, the effects of collisions between impurities and deuterons must be carefully examined. The ion velocity is no longer negligible compared to the electron velocity: the speed of a 10-keV deuterium ion is one-fifth that of a 60-eV electron, and the ion carries much more transferable momentum. The consequences $0:-$ this $f$ act for ionization and excitation rates are assessed in Appendix A. For most of the impurity ions in the plasma, these effects are unimport ant.

Finally, the plasma boundary is of interest because it can affect the loss of particles and energy from the plasma. A cold plasma layer may exist in 2XIIB [9], established by diffusion or gas reflux. The temperature and density profiles in this region are not well know. As is discussed in Appendix $H$, some clues may be obtained from the EUV data. 
D. Recent 2XIIB Operation

During the ten-month EUV study, several mirror physics experiments were performed by the 2XIIB staff. This section will mention those experiments, and will discuss the plasma parmeters which were produced. Details about recent 2 XILB plasma physics results and the 2 XIIB diagnostics may be found in references $[5,10-12]$.

2XIIB established and maintained plasmas with widely varying characteristics, which depended on the amount of beam current supplied, neutral-beam aiming, and other factors. The electron density varied from $10^{13} \mathrm{~cm}^{-3}$ to more than $10^{14} \mathrm{~cm}^{-3}$, and the electron temperature was as 1 ow as $30 \mathrm{ev}$ and at times exceeded $150 \mathrm{eV}$. Energy and particle confinement times near 1 ms were obtained. On the one hand, this wide variation allows us to observe important affects (see, for example, the discussion of 0 II emissions in Section $v$ ). On the other hand, we must consider such variations when analyzing data and drawing conclusions.

\section{Recent Experiments}

Substantial EUV data were obtained during three major 2XIIB experiments. From September to December, 1977, a field-reversal experiment was performed [11]. The neutral beams were aimed to minimize the plasma radius while maximizing its diamagnetic currents. The magnetic field was reduced to near zero in the center of the plasma but was not reversed.

Large-radius plasmas were studied from January to May 1978. The neutral beams were aimed to maximize the plasma radius. The large-radius plasmas obtained larger electron temperatures and energy confinement than comparable small-radius plasmas did [5]. This is apparently connected with better stabilization of the dominant drift-cyclotron, loss-cone instability. 
During May through July 1978, plasmas were created to study the mechanisms determining the electron temperature in 2XIIB. Unexpected gradients of electron temperature along magnetic field lines were measured and are now theoretically understood [12]. (These gradients gre not in the central plasma region observed during this study.)

Several other experiments were performed during this period, notably including an attempt to stabilize the plasma with electron beams [ 13]. Some EUV data vere obtained during these experiments, but they were in general too brief to yield substantial insight. 


\section{SPECTROSCOPIC INSTRUMENTATION AND THEORY}

\section{A. Introduction}

This section provides the spectrosconic background needed to place in perspective the results and arguments presented below. The spectrometer is described and its mounting on 2XIIB is explained. The theoretical concepts necessary to interpret the spectroscopic data are reviewed. In addition, a number of atomic processes are evaluated to determine which ones are important in the environment of the 2XIIB plasma.

\section{B. The Spectrometer}

The spectrometer used in this study is a $0.4-m$ normal incidence spectrophotometer that is described in detail elsewhere [14]. A brief description, including the modifications made for 2 XIIB, will be given here. The grating drive was designed by William G. Fastie of the Johns Hopkins University, rotates the $0.4-m$-radius-of-curvat ure concave grating with a sine-drive mechanism, to obtain a linear wavelength variation, while translating the grating to maintain good focusing (see Fig. 3). The entrance slit and exit slit are fixed in position, each set $4.3^{\circ}$ from the zero-order grating normal. The exit slit has a fixed height; the entrance slit uses a plate with a veeshaped notch to vary the slit height uniformly about its center. Both slits have one movable edge, which may be accurately set to give a slit width of 50 to $1300 \mu \mathrm{m}$.

The Bausch and Lomb grating is platinum coated, has $2400 \mathrm{lines/mm}$ and is blazed near $300 \AA$. This combination results in a dispersion of $10 \mathrm{~A} / \mathrm{mm}$. The experimentally determined resolution of the optics is $0.2 \stackrel{\AA}{A}$. To obtain reliable photometric accuracy, we must consider the $\pm 0.3-\AA$ wavelength 


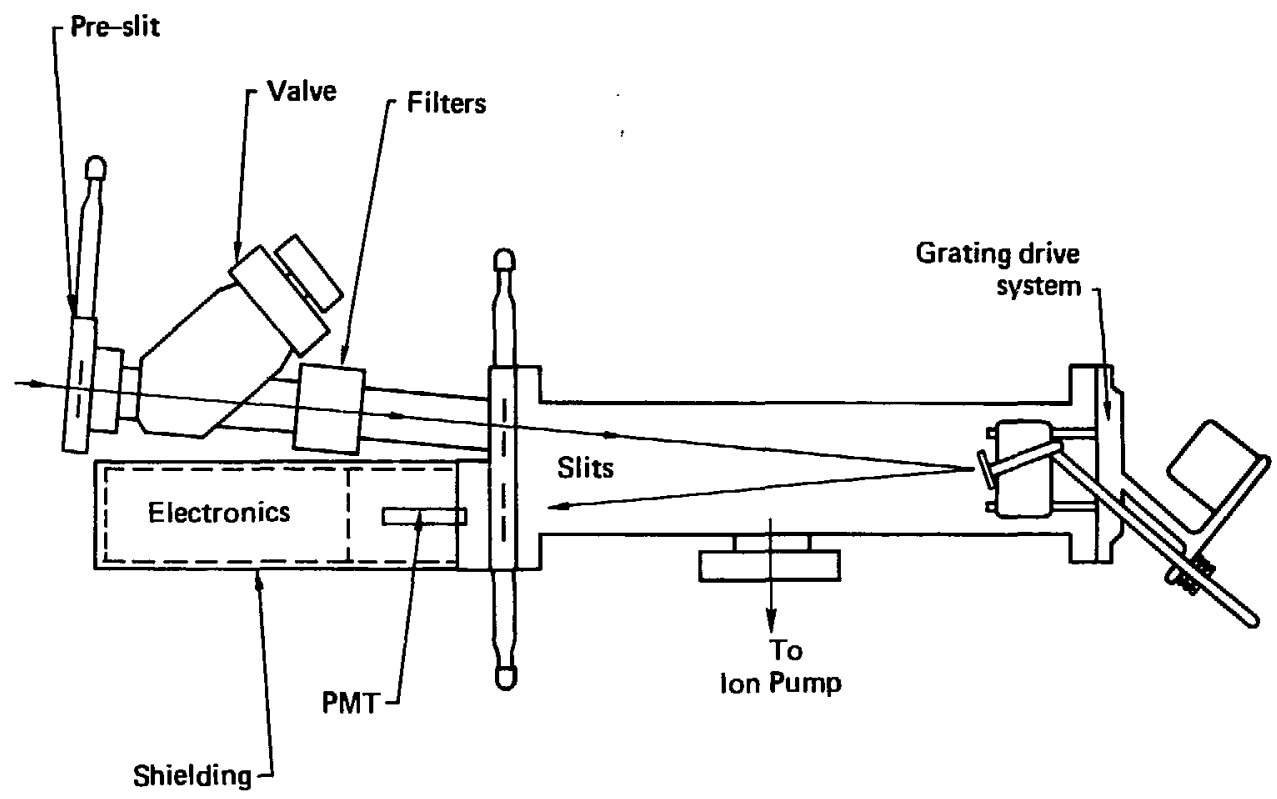

Figure 3. Schematic of the 0.4-m monochromator.

resettability of the grating drive, as it was mounted on $2 X I I B$, and the irreproducibility of the plasmas under observation. Under these restrictions, the usable resolution is about $2 \AA$ (and is greater if there is significant Doppler broadening).

The detector is an EMR $510 \mathrm{~W}$ windowless photomultiplier tube with a copperiodide coating that enhances its sensitivity at wavelengths above $1200 \AA$ [15]. Lead shielding protects the detector from $x$ rays, and $a$ magnetic shield was used to isolate it from the strong $(500-G)$ fields encountered near 2 XIIB. The shield uses several layers of material to reduce the field and overlapping cylinders of different radii to overcome end effects (see Fig. 4). It was tested to a 500-G longitudinal and 1000-G transverse field. 


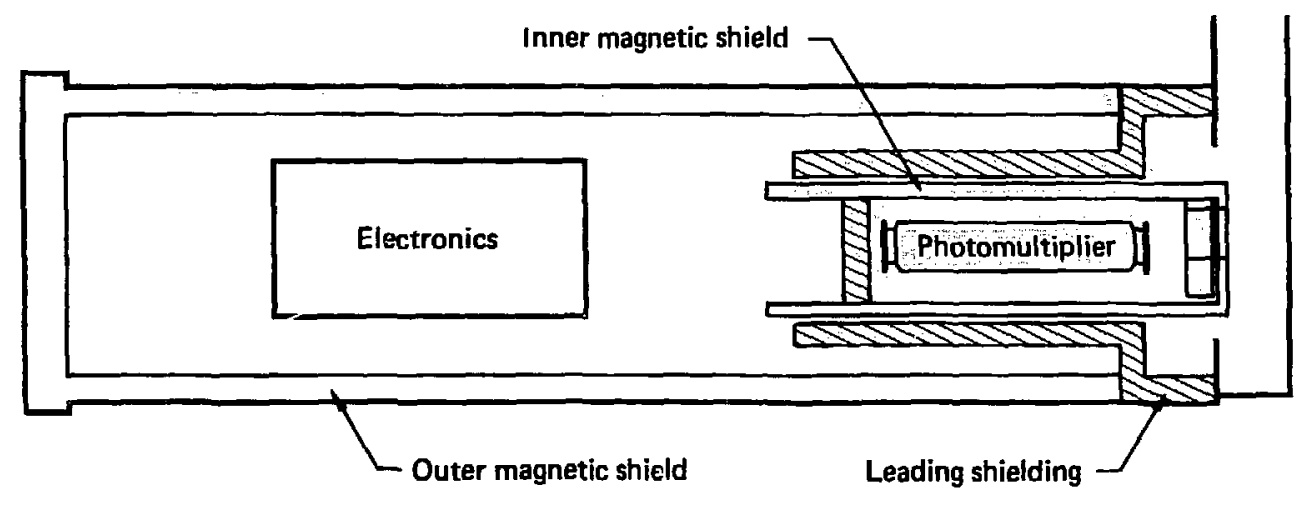

Figure 4. The magnetic shielding of the EUV detector. Note that the open ends of the inner and outer shields overlap, reducing field penetration through the ends.

The instrument was absolutely calibrated before and after the 2 XIIB experiment using the calibration facility at Johns Hopkins, which gives results consistent with those obtained using the National Bureau of Standards (NBS) calibration facility [14]. The calibration is performed first measuring the intensity of a monochromatic beam with an absolutely calibrated reference photodiode obtained from NBS [16], then measuring the signal obtained after a mirror is moved to pass the same beam through the instrument. The sensitivity of the instrument dropped up to $24 \%$ during the 2 XIIB experiment. In consequence, including the calibration uncertainties, the calibration is known to better than $\pm 22 \%$ over the primary spectral range used $(450-1220 \AA)$ and less well from 400 to $1600 \AA$. The good stability of the calibration is attributed to the continuous evacuation of the instrument by an ion pump throughout the 12-month experiment and to the use of copper gaskets for all demountable vacuum joints. 
The $0.4-m$ instrument was mounted on top of 2 XIIB, with the orientation shown in Fig. 1. The line of sight passed between the yin-yang coils and intercepted the central plasma. It was $13^{\circ}$ from the vertical and $3^{\circ}$ from the midplane of 2 XIIB. The entrance slit was $2.84 \mathrm{~m}$ from machine center.

The instrument was held by a carriage that could be titled by remote control. The pivot point was 2.37 m from machine center; and the motion was perperidicular to the magnetic axis. The line of sight could be moved more than $15 \mathrm{~cm}$ from the axis in eack direction, allowing the entire plasma to be scanned. A variable preslit allowed the field of view to be adjusted. As a result, the number of shots needed to scan the plasma could be matched to the number of shots available with constant plasma parameters.

During a run, the instrument and beam-line we re directly connected to the 2XIIB vacuum system. A shutter in the beam-1ine protected the instrument from the titanium emitted by the getter wires. Ten seconds before each shot, the shutter was opened. The ion pump on the instrument was also turned off, to keep it from affecting the signal. After the shot, the shutter was closed, and the pump was turned on.

\section{Atomic Processes}

Understanding and interpreting spectroscopic data require knowledge of the atomic processes that occur in the plasma being studied. This section reviews the relevant atomic processes and indicates which ones are important here. Its significant conclusion is that charge-exchange is the dominant recombination process in the central 2XIIB plasma, which is seldom true in tokamaks. The important processes are distinguished by comparing the rates 
at which various processes occur, so a discussion of lifetimes and rates foll ow.

The rate $v$ and lifetime $\tau$ for a process resulting in a change in some quantity $x$ are defined by:

$$
v=1 / \tau=\frac{1}{x} \frac{d x}{d t}
$$

If $\tau$ is not a function of $x$, it is the characteristic time for exponential growth or decay of the quantity $x$. The processes of interest most often occur when a target atom is bombarded by another particle. In this case the lifetime is:

$$
\tau=\left(n_{p}<\sigma v>\right)^{-1}
$$

in which $n_{p}$ is the density of the bombarding particles and $\langle\sigma \mathrm{v}\rangle$ is the average of the product of the cross section for the process and the relative velocity of the collision. Tables 1 and 2 sumarize the characteristic lifetimes of the important atomic processes in 2XIIB. These processes will be considered and evaluated in more depth below. Energies are in keV, masses are in amu, and other quantities in cgs units. The sources of rate coefficients are described in the text.

\section{Emission-Related Processes}

Because the spectrometer measures radiation intensity, processes which pertain to emission are of first importance. The volume enission rate is

$$
E=n * A \text { photons } \mathrm{s}^{-1} \mathrm{~cm}^{-3}
$$

in which $n *$ is the density of excited atoms and $A$ is the Einstein coefficient for spontaneous emission, of order $10^{9} \mathrm{~s}^{-1}$. Spontaneous decay is in general 
TABLE 1. IMPURITY TIME SCALES*

\begin{tabular}{|c|c|c|c|c|c|c|c|c|}
\hline \multirow[t]{2}{*}{ PROCESS } & \multirow[b]{2}{*}{ OI } & LIFETIME & E OF STATE & \multirow{2}{*}{$\frac{\text { FOR PROCESS }}{\text { OIV }}$} & \multicolumn{2}{|c|}{ IN MICROSECONDS } & \multirow[b]{2}{*}{ OVII } & \multirow[t]{2}{*}{ REFERENCE } \\
\hline & & $\mathrm{OTI}$ & OIII & & OV & OVI & & \\
\hline Ionization by $e^{-* *}$ & 0.24 & 0.84 & 2.4 & 6.4 & 22 & 77 & $3.3 \times 10^{5}$ & 24 \\
\hline $\begin{array}{l}\text { Charge Exchange on } \\
\text { Neutral Deuterium }\end{array}$ & 0.29 & 270 & 910 & 33 & 40 & 50 & 63 & 26,27 \\
\hline Spitzer Drag & & 6700 & 1700 & 750 & 420 & 270 & 190 & 29 \\
\hline $\begin{array}{l}\text { Dielectrs tic and } \\
\text { Radiativ, Recombination }\end{array}$ & & $1.6 \times 10^{5}$ & $8 \times 10^{4}$ & $2 \times 10^{4}$ & $4 \times 10^{4}$ & $2.5 \times 10^{5}$ & $1.2 \times 10^{5}$ & 19 \\
\hline Scattering of 0 by 0 : & & & & & & & & \\
\hline 100-ev Oxygen & & 1900 & 120 & 24 & 7.8 & 3.0 & 2.5 & 30 \\
\hline 10-kev Oxygen & & $1.9 \times 10^{6}$ & $1.2 \times 10^{5}$ & $2.4 \times 10^{4}$ & $7.8 \times 10^{3}$ & $3.0 \times 10^{3}$ & $1.5 \times 10^{3}$ & \\
\hline Scattering of 0 by $\mathrm{D}^{+}$: & & & & & & & & \\
\hline 100-ev Oxygen & & 1100 & 280 & 120 & 69 & 45 & 30 & \\
\hline 10-kev Oxygen & & $1.1 \times 10^{5}$ & $2.8 \times 10^{4}$ & $1.2 \times 10^{4}$ & $6.9 \times 10^{3}$ & $4.5 \times 10^{3}$ & $3 \times 10^{3}$ & \\
\hline
\end{tabular}

*The foll owing parameters are ass umed

$\mathrm{T}_{\mathrm{e}}=100 \mathrm{ev}, \mathrm{n}_{\mathrm{e}}=5 \times 10^{13} \mathrm{~cm}^{-3}, \mathrm{n}_{\mathrm{D}}=5 \times 10^{10} \mathrm{~cm}^{-3}, \mathrm{v}_{\mathrm{D}}=10^{8} \mathrm{~cm} \mathrm{~s} \mathrm{~s}^{-1}, \mathrm{n}_{0}=10^{12}$

**F or comparison, excitation times for strong lines are typically $1 \mu \mathrm{s}$. 


$$
\begin{aligned}
& \text { Process } \\
& \begin{array}{l}
\text { Charge } \\
\text { Exchange }
\end{array} \quad=\frac{1}{n_{0}^{<} \sigma_{v}>c x} \text { (ions) } \\
& =\frac{1}{n_{e}<v>^{c x}} \text { (neutrals) } \\
& \text { Spitzer Drag } \tau=\frac{10^{13} \mathrm{MT}_{\mathrm{e}}^{3 / 2}}{\mathrm{n}_{\mathrm{e}} \ln \Lambda \mathrm{z}^{2}} \\
& \text { Recombination } \tau=\frac{1}{n_{e}^{<} \sigma v>^{R E C}} \\
& \text { Scattering } \quad n_{b} \tau_{a b}=5 \times 10^{10} E_{a} E_{b}^{1 / 2} A_{a} A_{b}^{-1 / 2} z_{a}^{-2} z_{b}^{-2} \log _{10} R_{n}
\end{aligned}
$$


much faster than collisional deexcitation or stimulated emission. It is also much faster than all the excitation processes, which have maximu rates of order $10^{6} \mathrm{~s}^{-1}$. This has two consequences: At any given time, almost all the particles of one type are in the ground state (metastable systems are discussed below), and the emission rate is determined by the excitation rate. Electron impact excitation is in general the dominant excitation process. Photon excitation is not important. Excitation as a result of recombination or ionization into an excited state may be important for transitions among excited states [17], but is unimportant for the ground-state resonance transitions observed here. Ion impact excitation is in general slow compared to electron impact excitation. Some exceptions may occur for transitions in low ionization states, as a result of the high ion temperature of 2XIIB. This issue is discussed in Appendix A.

Given that electron impact excitation determines the emission rate, Eq. (3) can be rewritten:

$$
E=n_{e^{N}\langle\sigma v\rangle^{e x}}
$$

The electrons of density $n_{e}$ excite the ions of density $N$, with a rate coefficient $\left\langle\sigma_{v}\right\rangle^{e x}$. The rate coefficients used here were obtained from recent calculations [18-22]. The calculations general1y agree quite well with one another. In cases of disagreement, the close coupling calculations of Robb [18] were used.

Many impurity atoms have metastable systems which cannot decay rapidly to the ground state. For example, the $1 s^{2} 2 s^{2} \mathrm{p}^{3} \mathrm{p}$ state of Be I (or $0 \mathrm{v}$ ) decays very slowly to the $1 s^{2} 2 s^{2}{ }^{1} s$ ground state $\left(A \sim 10^{3} s^{-1}\right.$ ) [23]. These systems are populated as a result of excitation, ionization, and recombination processes. Their populations may exceed those of the ground-state 
systems; they may be determined by measuring resonance transitions from the lowest metastable state, and must be considered when impurity concentrations and power loss are evaluated.

\section{Ionizing and Recombing Processes}

The atoms and ions in the plasma change their ionization state by ionization and recombination (including charge-exchange). Electron impact ionization is the dominant ionizing process for most ions. Neutral atoms may become ionized by charge-exchange on deuterium ions:

$$
A+D^{+} \rightarrow A^{+}+D^{0}
$$

This process is effective $f$ or neutral atoms in $2 \times I I B$, but is not important For multiply ionized impurities. This is because the tightly bound electrons move too quickly to effectively gain energy from the slower deuterium ions. Neutrals and ions in low-ionization states may also be affected by deuteronimpact ionization, as assessed in Appendix A.

The ionization rate coefficients used here are taken from Lotz [24] . The measured values tend to be somewhat lower than these tabulated values, as L. A. Jones discussed during a recent conference [25]. This fact is considered in section $v$, during the detailed analys is of the oxygen data.

Charge-exchange was the most important recombination process in the central 2XIIB plasmas, as a result of the relatively large density of highenergy neutrals discussed above. As may be seen in Table 1, radiative and dielectronic recombination are typically 1000 times slower than chargeexchange. Collisional recombination, a three body process, is unimportant because the electron density is too low.

The cross sections for charge-exchange, 


$$
\mathrm{A}^{2}+\mathrm{D}^{0} \rightarrow \mathrm{A}^{\mathrm{2}-1}+\mathrm{D}^{+},
$$

are taken from recent measurements $[26,27]$. These measurements were performed at relative velocities close to those present in 2 XIIB, and the cross sections are believed to vary slowly with velocity over the range that is important for $\langle\sigma v\rangle[2 \%]$. (This is because the velocity distribution in 2XIIB has few ions at very high or very low velocities [7].) In consequence, the rate coefficients were computed as:

$$
\langle\sigma \mathrm{v}\rangle \cong \bar{v}=10^{8} \quad \sigma \mathrm{cm}^{3} \mathrm{~s}^{-1}
$$

The averaging is estimated to be accurate within $\pm 20 \%$.

\section{Scattering and Heating Processes}

In a mirror machine, the velocity and energy of confined particles must satisfy certain requi rements (see section II). As a result, processes that heat the ions or scatter them in velocity space are important. Coulomb collisions can lead to both scattering and heating, as is discussed below. Radio-frequency (rf) fluctuations can also scatter and heat particles in 2XIIB. These effects are important but are not in general dominant for the deuterium in the plasma $[7,28]$ (the energy confinement is governed by Coulomb effects). The deuterium ions are resonant with the deuteriun ion cyclotron radiation that dominates the $\mathrm{rf}$ fluctuations. As the impurity ions are not resonant with this radiation, rf effects should be less important for them. In general, such effects will be ignored below.

It is well known that the important Coulomb effects in flasmas are cumulative ones, resulting from many collisions at large impact parameter [29] . As an illustration, using typical 2 XIIB parameters, the cross section for 
collisions closer than a Debye length is of order $10^{-7} \mathrm{~cm}^{2}$ vs a cross section of about $10^{-21} \mathrm{~cm}^{2}$ for collisions resulting $2 n$ deflections greater than $90^{\circ}$. The D+D fusion cross-section at $10 \mathrm{keV}$ is $10^{-26} \mathrm{~cm}^{2}$. The effects of many small angle collisions are best computed using the Fokker-Planck equation.

Scattering changes the direction of a velocity vector; but it should only be important for low-energy, poorly-confined impurities in 2XIIB. Its effects are most often expressed in terms of a "90" scattering time," which is the average time required for the velocity vector to be shifted in direction by $90^{\circ}$. Computer simulations are in general consistent with analytic estimates, as discussed by Futch et al. [30]. The $90^{\circ}$ scattering time may be estimated as:

$$
\mathrm{n \tau}_{\mathrm{ab}}=1.25 \times 10^{10} \mathrm{E}_{\mathrm{a}} \mathrm{E}_{\mathrm{b}}^{1 / 2} \mathrm{~A}_{\mathrm{a}} \mathrm{A}_{\mathrm{b}}^{-1 / 2} \mathrm{z}_{\mathrm{a}}^{-2} \mathrm{z}_{\mathrm{b}}^{-2}
$$

Energies are expressed in $\mathrm{keV}$ and masses in amu, with $\mathrm{nt}$ in $\mathrm{cm}^{-3} \mathrm{~s}$. Equation (5) gives the average time required for a $90^{\circ}$ deflection of a particle of type $\underline{a}$, as a result of collisions with particles of type $\underline{b}$. The global mirror machine confinement time, if ion losses are dominated by scattering, is given by $\mathrm{Eq}$. (5) times a factor $4 \log _{10} R_{m}$, in which $R_{m}$ is the mirror ratio [30]. The latter quantity was included in the lifetimes found in Table 1 (its value is 1.2 for 2 XIIB). Some typical scattering lifetimes for oxygen in 2XIIB were given in Table 1 . Scattering is seen to be important only for low-energy impurities, which are al ready poorly confined because of the positive plasma potential. Thus, scattering should not significantly affect impurity confinement in the central plasma.

Heating of cold impurities by the hot ions in the plasma must also be evaluated. If cold impurities, with barely enough energy to pass through the 
plasma, can be heated (and, in consequence, confined) within one transit time, a much smaller impurity influx is required to obtain a given concentration of impurities. Simple estimates, based on the standard formulas of Spitzer [29], suggest that such heating times are far too long to matter. However, because these formulas were obtained using a Maxwellian velocity distribution, they do not literally apply to 2XIIB. A better calculation, based on the Fokker-P1anck equation and using a standard model of the 2XIIB velocity distribution [ 7 l, also showed that cold impurities are negligibly heated by collisions with the hot ions.

E. Measurement of Brightnesses, Densities and Concentrations

This section discusses the measured EUV brightness, and the relation of the impurity densities and concentrations to it. The interpretation of EUV spectroscopic data is model-dependent, and the models to be used should be determined by direct study of the plasma being modeled. In this study, much effort was expended to ensure that good models were used and that the conclusions were not artifacts of the models.

\section{Brightnesses}

The quantity measured with this equipment is monuchromatic surface brightness. The brightness of emissions from an optically thin source is related to the volume emission rate $E(\underline{r})$ by

$$
\mathrm{B}=\frac{1}{4 \pi} \int \mathrm{E}(\underline{\mathrm{r}}) \mathrm{d} 1 \text { photons } \mathrm{s}^{-1} \mathrm{~cm}^{-2} \mathrm{sr}^{-1},
$$

in which $\mathrm{E}(\underline{\mathrm{r}})$ is in units of photons $\mathrm{s}^{-1} \mathrm{~cm}^{-3}$ into $4 \pi$ steradians and the integral is taken along the line of sight of the measuring instrument. 
The brightness was resuted to the measured detector current as follows:

$$
B=\frac{I}{e Q T G} \frac{a^{2}}{A_{s} A_{p s}},
$$

in which $I$ is the observed current, QTG is the net gain, of the spectroscopic system, $a$ is the distance from entrance slit to preslit, and $A_{s}$ and $A_{p s}$ are the areas of the two slits. The last two quantities applied because there were no smaller optical stops in the system. Equation (7) may be derived by integrating the signals due to emission from each elemental volume throughout the field of view.

We can now obtain a relation between the measured monochromatic surface brightness of the plasma and the microscopic electron and impurity ion densities. The substitution of Eq. (4) into Eq. (6) yields

$$
B=\frac{1}{4 \pi} \int n_{e} N<\sigma v>x_{d 1} .
$$

The transitions observed have energies on the order of $15 \mathrm{eV}$. In such cases, $<\sigma v>^{e x}$ varies slowly with $\mathrm{T}_{\mathrm{e}}$ above $20 \mathrm{eV}$. In consequence, $<\sigma_{\mathrm{v}}>^{\mathrm{ex}}$ can usually be withdrawn from the integ:al.

\section{Densities and Concentrations}

Given $\mathfrak{n}_{e}(\underline{r}), T_{e}(\underline{r})$, and the spatial profile of the impurity ions, Eq. (8) can be integrated to obtain the impurity density of an ionization state. If this can be done for all ionization states of a given impurity species, the total density of that species and hence its concentration in the plasma are known.

As a practical matter, the impurity spatial profiles are not wellknown, hence we use simple models, three of which are mentioned here: the 
constant-density (flat profile), constant-concentration, and thin-shell models. If the impurities are evenly distributed - the constant-density model $-\rightarrow$ and the $T_{e}$ profile is sufficient $1 y$ broad, both $N$ and $\langle\sigma v\rangle^{e x}$ can be removed from the integral in Eq. (8), yielding

$$
N=\frac{4 \pi B}{\langle\sigma v\rangle^{e x} \int n_{e} d 1} c m^{-3}
$$

Spatial scans and Thomson scatering data indicate that this is usually the appropriate model for 2 XIIB. In the constant concentration model, the $\mathbf{n}_{e}$ and $N$ profiles are proportiona1 (and $\langle\sigma v\rangle^{e x}=$ const.), thus Eq. ( 8 ) can be integrated di rectly because the electron density profile is known. Finally, if the impurity ions exist in a shell at $r=a, E q$. (8) becomes

$$
B=\frac{1}{4 \pi} n_{e} \text { (a) } N<\sigma v>^{e x} 2 \Delta w,
$$

where $\Delta \mathrm{w}$ is the shell thickness. 


\section{A. Introduction}

The lack of detailed knowledge about impurities in mirror machines meant that it was impossible to anticipate EUV brightnesses or emission characteristics. In consequence, a major survey was necessary to determine the general characteristics of EUV emissions from 2XIIB. The concentrations of impurities, and the power radiated by them, could be estimated once the EUV emission characteristics were known. This section sumarizes th: characteristics of the EUV emissions from 2 XIIB and the conclusions following from them.

Four imp.rity species, of which oxygen was most abundant, were identified in 2XIIB. The time developments, brightnesses, energies, and spatial distributions of EUV emissions are summarized in the next section of this section. The final section sumbizes the implications of the EUV data for impurity concentrations and radiated power 1 oss.

The oxygen data will be emphasized throughout, in preparation for the detailed discussions of Section $V$, which present the results of an extensive investigation of the oxygen emissions from 2XIIB. Appendix C contains a detailed account of the survey of EUV emissions. Appendix F evaluates the possible carbon sources in 2 XIIB on the basis of the carbon data. It also summarizes what little is known about the sources of titanium and nitrogen.

\section{B. EUV Emissions from 2XIIB}

Emissions of oxygen titanium, carbon, and nitrugen were identified. Shct-to-shot spectral scans under constant machine conditions were used to identify emission 1ines, with the aid of the wavelength calibration of the 
spectrometer and standard tables of atomir transitions [31]. The observed transitions are tabulated in Appendix $c$, and the line identification is discussed more thoroughly there. Most of the transitions observed on 2XIIB had been observed previously on Alcator [32], using the same equipment, which increases the reliability of the line identifications.

During the first $f$ ew months of this EUV study, the time development of EUV emissions from 2 XIIB was observed, and it came to be understood. It did not change dramatically throughout the entire study. Typical time histories of five oxygen states are presented in Figs, 5 and 6 . The emission characteristics will be described with reference to these figures.

The time development of the 2 XIIB discharge can be divided into three phases: the target plasma buildup, the hot plasma buildup, and the highdensity plateau. During the initial experiments, a target plasma was provided by titanium washer guns beginning at 1.2 ms after machine "zero time." The hot plasma buildup began when the neutral beams fired at $1.8 \mathrm{~ms}$ and ended when the high density plateau was reached at about 4 ms. The plateau lasted until about $8 \mathrm{~ms}$, depending on the duration of the neutral-beam current. During the target plasma buildup, low-intensity impurity emissions were observed from low ionization states. Emissions of 0 II and 0 III were observed by $1.4 \mathrm{~ms}$, and $0 \mathrm{IV}$ was observed by $1.8 \mathrm{~ms}$. The emissions at this time were so dim that they required measurement on more sensitive scale than that shown in Fig. 6.

A strong increase in EUV impurity emissions cccurred during the hot plasma buildup. Brightness peaks were observed from 0 IV, $0 \mathrm{~V}$, and sometimes 0 VI. These are believed to have resulted from the ionization of 0 II and 0 III ions already existing in the target plasma. They were characteristic of high-temperature plasmas with rapid buildup to high densities, but were 


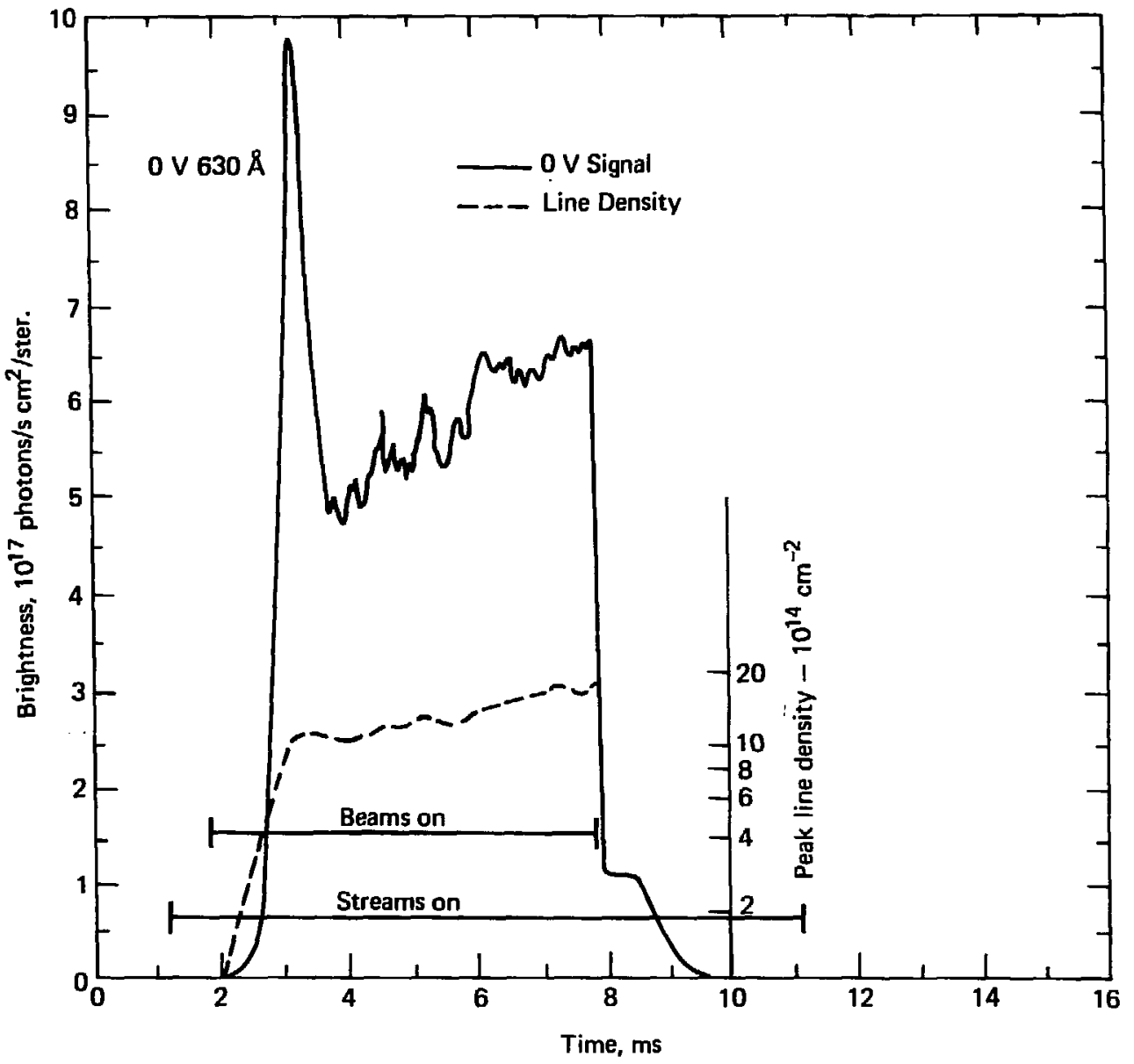

Shot 25 10/24/77

Parameters at $6 \mathrm{~ms}$

$\mathrm{T}_{\mathrm{e}}$

Radius

Line Density

Magnetic Moment

Neutral-Beam Current

Mirror Ratio
$175 \mathrm{eV}$

$\therefore \mathrm{keV}$

$9.5 \mathrm{~cm}$

$1.5 \times 10^{15} \mathrm{~cm}^{-2}$

$1600 \mathrm{~A}-\mathrm{m}^{2}$

$510 \mathrm{~A}$

2

Figure 5. A typical o V $630 \AA$ A time development. The electron line density (dashed $1 i n e$ ) and the beam and stream durations are al so shown. Some plasma parameters are given. 


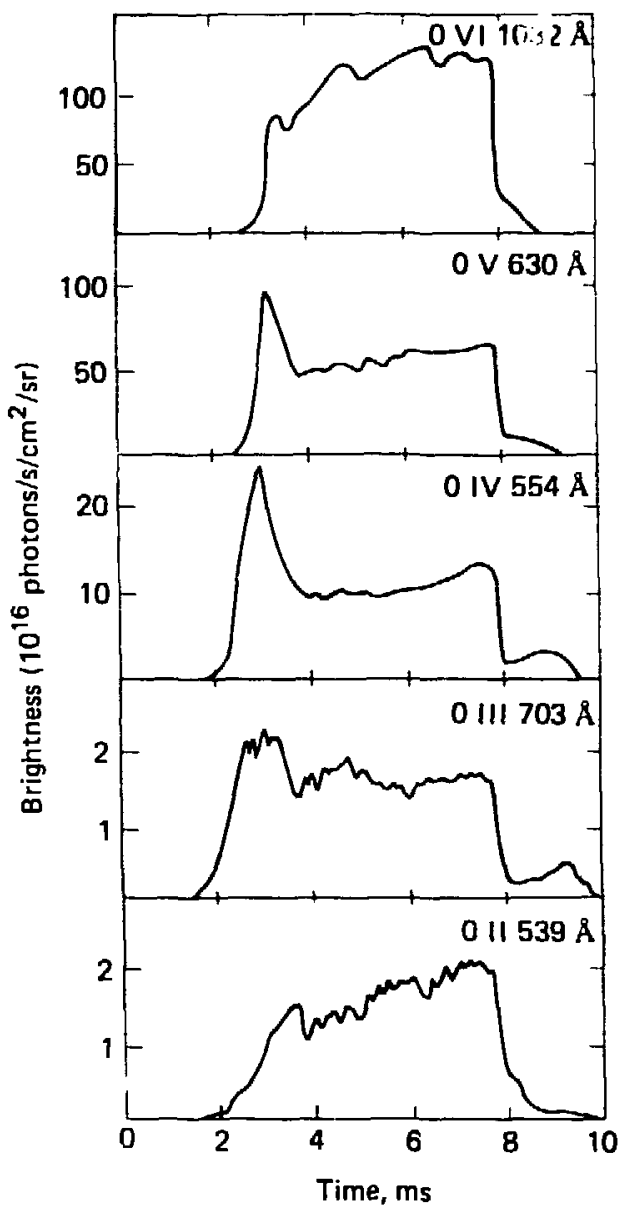

Figure 6. Typical emissions of the observed oxygen ions, from identical shots on 24 October 1977. 
not present in all of the plasmas studied. The successive appearance of emissions from ever higher ionization states lends additional support to the line identifications .

Impurity emissions showed some variations during the high-density plateau. Emissions from low ionization states often increased during this period, while emissions from higher ionization states exhibited variable changes. These variations were usually less than a factor of 2 .

The oxygen emissions were the brightest observed from 2XIIB. As Eig. 6 shows, 0 V $630 \AA$ and 0 VI $1032 \AA$ had brightnesses near $10^{18}$ ph $\mathrm{s}^{-1} \mathrm{~cm}^{-2} \mathrm{sr}^{-1}$. These enissions are very bright in comparison with those observed from other plasma devices. The O VI brightness is an order of magnitude greater than that reported from TFR [33], and is one hundred times levels observed on Alcator [32]. In addition, for 2XIIB plasmas of comparable density, the oxygen brightnesses seldom varied more than a factor of 2 throughout this study. These long-term variations are documented and discussed in Appendix C.

Spatial and spectral scans of EUV emissions were limited by the number of shots available with constant machine parameters. In general, the impurities were distributed throughout the plasma. Figure 7a shows the results of a spatial scan of $0 \mathrm{~V} 630-\AA$ emissions. The spatial $0 \mathrm{~V}$ brightness data were obtained on a shot-by-shot basis. The electron-line density profile was measured every shot. It did not vary substantially $( \pm 10 \%)$ and was fit well as a Gaussian profile. The average of these line density profiles is shown in Fig. 7. Figure $7 b$ shows the Abel inversion of the hand-drawn best fit to the $0 \mathrm{~V}$ spatial data, as well as two possible models of the spatial profile. Although the data are not accurate enough to precisely determine the $0 \mathrm{~V}$ 


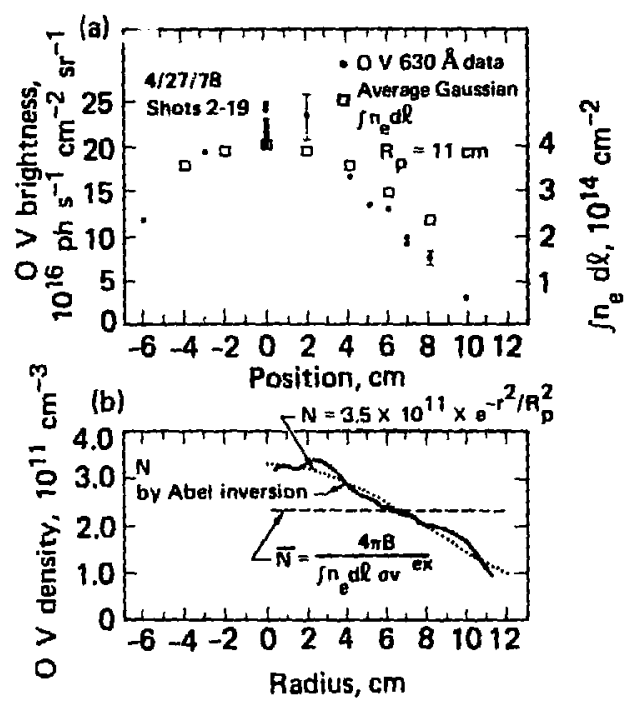

Figure 7. (a) o V $630-\AA$ brightness and el ectron line density profiles are shown. (b) $0 \mathrm{~V}$ density profiles given by Abel inversion and two models are shown; see text for details.

spatial distribution, the Abel inversions of various brightness profiles allowed by the data show that the constant concentration model (Section III D), which is the dotted curve in the figure, should be used for oxygen. The Gaussian profile of the $\mathrm{OV}$ density is proportional to that of the electron density. The dashed curve in the figure represents the constant density model; it is clearly a poorer fit. Other spatial data are discussed in Appendix $C$ and $D$.

The energy of the emitting impurity ions could be estimated on the basis of some of the spectral scans. These scans are documented and eva1uated in Appendix $C$, and are sumarized in Table 3. Table 3 presents the impurity energies corresponding to emission at the full width of half maximum 


\begin{tabular}{|c|c|c|c|c|c|c|c|}
\hline & $\begin{array}{l}\text { Observed } \\
\text { ransition }\end{array}$ & $\begin{array}{l}\text { Observed } \\
\text { FWHM } \\
\text { Above } \\
\text { Background }\end{array}$ & $\begin{array}{l}\text { slit } \\
\text { FWHM }\end{array}$ & $\begin{array}{l}\text { Actual } \\
\text { FWHM }\end{array}$ & & $\begin{array}{c}-=\text { HWHM } \\
\left(10^{-3}\right)\end{array}$ & $\begin{array}{c}\text { Energy at } \\
\text { FWhM } \\
\text { (kev) }\end{array}$ \\
\hline 0 & VI $1032 \AA$ & $3.5 \pm 0.7 \AA$ & $1.1 \AA$ & $3.3 \pm 0.7$ & $\AA$ & $1.6 \pm 20 \%$ & $19 \pm 40 \%$ \\
\hline 0 & V $630 \AA$ & $2.0 \pm 0.3 \AA$ & $1.1 \AA$ & $1.7 \pm 0.4$ & A & $1.3 \pm 25 \%$ & $13 \pm 50 \%$ \\
\hline 0 & $760 \AA$ & $2.4 \pm 0.5 \AA$ & $1.5 \AA$ & $1.9 \pm 0.7$ & $\AA$ & $1.3 \pm 37 \%$ & $13 \pm 74 \%$ \\
\hline C & III $977 \stackrel{\circ}{\text { A }}$ & $2.3 \pm 0.3 \mathrm{~A}$ & $1.1 \mathrm{~A}$ & $2.0 \pm 0.35$ & $\AA$ & $1.0 \pm 18 \%$ & $5.6 \pm 36 \%$ \\
\hline
\end{tabular}

(FWHM) of the observed lines. The details of Table 3 are also explained in the Appendix.

The oxygen in 2XIIB is clearly energetic, having a mean energy near $10 \mathrm{keV}$ (the mean energy is somewhat less than the energy at the half maximum of the observed line). This is high enough for the oxygen to be wellconfined, as is discussed in section $V$. The carbon energy is lower than that of the oxygen, but some of the energetic carbon could be confined in the central plasma. These observed energies are important clues for identifying impurity sources.

\section{Concentration and Power Loss}

In consequence of the measurements of EUV emissions from 2XIIB, it was possible to estimate the impurity concentrations using the methods described in Section III. The details of the brightness measurements, the uncertainties in these, and the choice of spatial models are discussed in Appendix C. The data that were analyzed in detail and the specific results obtained are tabulated there. Table 4 presents a summary of the impurity concentrations. 
TABLE 4. SUMMARY OF IMPURITY CONCENTRATIONS

\begin{tabular}{lcc}
\hline Species & $\begin{array}{c}\text { Concentration } \\
\%\end{array}$ & $\begin{array}{c}\text { Uncertainty } \\
\text { Factor }\end{array}$ \\
\hline Oxygen & $3 \%$ & 2 \\
Carbon & $0.4 \%$ & 2.5 \\
Nitrogen & $0.5 \%$ & 3 \\
Titanium & $0.3 \%$ & 4 \\
\hline
\end{tabular}

0xygen is apparently much more abundant than the other impurities, but carbon, nitrogen, and titanium all exist in nonnegligible quantities. As Section $V$ shows, the oxygen concentration is reasonable because the oxygen is injected by the neutral beams and is well-confined. But, as is discussed in Appendix F, the origins of the carbon and titanium are not understood. The radiated power from the 2XIIB plasma was estimated on the basis of the observed brightnesses. About $50 \mathrm{~kW}$ of power is radiated by the impur ities in 2 XIIB (to a factor of 2), of which $80 \%$ is due to the oxygen. The observed radiated power per unit volume $\left(\sim 10 \mathrm{w}_{-\mathrm{cm}^{-3}}\right)^{3}$ is high compared to most tokamak results $\left(\sim 1 \mathrm{~W}-\mathrm{cm}^{-3}\right)[32,33]$. However the power input to 2 XIIB is so high ( $\left(300 \mathrm{~W}-\mathrm{cm}^{-3}\right)$ that the impurity radiation is an insignificant power loss ( $\sim 5 \%)$. Moreover, the impurities affect the 2 XIIB power balance in other ways, as a result of scattering and charge-exchange. The effects of oxygen in this regard are discussed in section V-D. The method by which the radiated power loss was calculated and the specific results obtained are included in Appendix c. 


\section{RESULTS OF A DETAILED STUDY OF OXYGEN EMISSIONS}

\section{A. Introduction}

This section presents the results of the detailed investigations of oxygen emissions from 2XIIB, which were undertaken after the unexpected discovery of a few percent of highly ionized oxygen. The natural questions of how such oxygen could penetrate the plasma and be confined were pursued, and in time several conclusions were reached.

The source of the oxygen impurity is shown to be the neutral beams in section B. This has implications for the oxygen confinement, whicil allows a model of the equilibrium distribution of oxygen ions anong different ion ization states to be developed in section $C$. This model, which assumes a balance of ionization and charge-exchange, is consistent with the data. Fiarlly, the resulting understanding of oxygen characteristics allows a more thorough evaluation of its effect on the plasma power balance, which includes several important processes in addition to radiation.

B. Evidence for Neutral-Beam-Injected Oxygen

This section argues that the principal source of oxygen in 2 XIIB is the neutral beams. All the available evidence supports this hypothesis, and there is evidence which conflicts with any other reasonable one. The most dramatic evidence is the strong dependence of the 0 II 539 A emissions on beam current; this dependence has been successfully modeled by assuming beaminjected oxygen. Spatial and spectral scans of oxygen emissions, impurity injection experiments, and other experiments also support this conclusions.

The 0 II emissions showed a strong dependence on beam current. The peak signal from the 0 II $539-\AA$ multiplet was measured on two days as the 
neutra1- «am current was systematically varied from 150 equiv A to $500 \mathrm{~A}$ on a shot-by-shot basis. Figure 8 shows the $O$ II signal as a function of beam current from one such scan. This data has been corrected for a s-gnificant background signal (about half the total signal of the peak of the $539-\AA$ feature), which was carefully measured during this scan. The best power-law fit to the 0 II data in Fig. 8 is that the $O$ II brightness increases as beam current to the 1.6 power. The line density is roughly proportional to beam current. The C III $977 \AA$ data, obtained during another scan, shows that impurity emissions do not, in general, behave like the 0 II emissions.

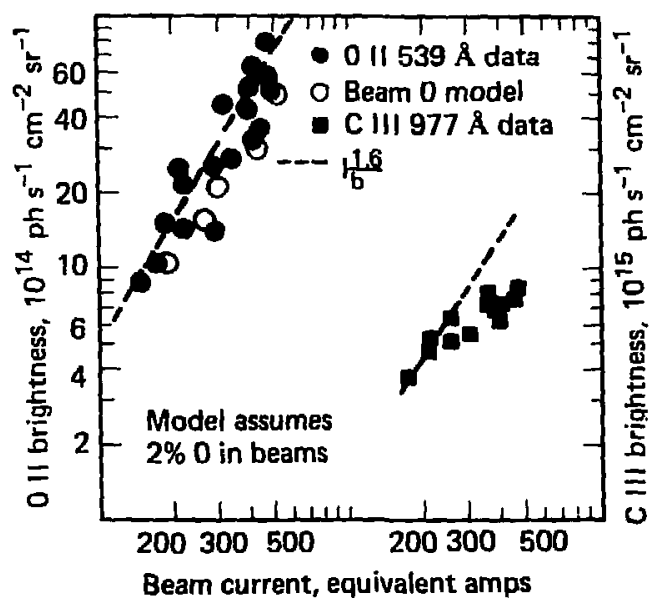

Figure 8. The 0 II brightness was observed to scale as the neutral-beam current to the 1.6 power, and it was successfully modeled. C III emissions scale differently. 
This strong dependence of oxygen signal on beam current is expected if the beams are the source of oxygen. The 0 II emissions are approximately proportional to the rate at which oxygen is trapped in the plagma. This is because both ionization and excitation rates are proportional to the electron density. Thus, the average number of photons emitted by each 0 II ion before it changes ionization state is independent of electron density; it depends only weakly on $\mathrm{T}_{e}$. The rate at which neutral-beam oxygen atoms are trapped by the plasma depends on the product of the oxygen current (probably a fixed percentage of the beam current) and the attenuation of that current by the plasma, which increases when the plasma line density does. This leads to a greater-than-1inear dependence of oxygen trapping and hence of 0 II emissions on beam current.

The strong dependence of 0 II brightness on beam current is thus important, independent of the details of any particular model; but the 0 II brightness resulting from beam injected oxygen can be modeled. A quantitative model has been constructed, which determines an 0 II brightness based on the trapping and ionization of neutral-beam oxygen. It predicts that the 0 II brightness should scale as beam current to the 1.55 power, which is consistent with the data. According to this model, the observed brightness corresponds to roughly a $2 \%$ particle concentration of oxygen in the beams. The predictions of the model are shown in Fig. 8. It is derived in Appendix E. By evaluating the relative trapping and lifetimes of 0 II ions and deuterons, the average 0 II density is found to be

$$
\vec{n}_{0 I I}=f_{0} \frac{1-T_{0}}{1-T_{D}} \frac{\left\langle\sigma_{v}\right\rangle^{c x}}{\left\langle\sigma_{v}\right\rangle_{0 \text { II }}^{\text {ion }}} n_{D} \propto I_{b}^{0.5}
$$


in which $f_{0}$ is the fraction of oxygen in the beams, $T_{0}$ and $T_{D}$ are the average transmissions of oxygen and deuterium through the plasma, and $n_{D}$ is the neutral deuterium density. The brightness is then given by Eq. (9) in Section III, and is proportional to the 1.5 power of the beam current. (The uncertainty in the exponent is about $\pm 15 \%$ )

If the oxygen was beam injected, its transverse velocity should have been correspondingly high. This was true, as was discussed in section IV (see Table 3). The spectral data and detailed analysis are in Appendix $\mathrm{C}$ (Figs. C3 and C4). On the basis of the observed Doppler broadening, the oxygen energy was estimated to be $10 \mathrm{keV}$. This energy could result from beam injection of high-energy oxygen, but is unlikely to result from slow collisional heating; the lifetime of low-energy impurities is too short.

The possibility that the oxygen ions were anomalously heated is eliminated by the 0 II spatial data, which requires that the neutral oxygen enter the plasma at high energies. This is shown by the spatial distribution of 0 II emissions, which did not show a well-defined shell structure. Background subtracted 0 II 539 A spatial data is presented in Fig. 9. Linedensity data fron the same run and some of the data analysis are also shown. The spatial data is limited by the availability of shots with constant machine parameters. This data does not allow a precise determination of the 0 Il density profile by Abel inversion. However, the inversions of curves drawn through the data may be used to place limits on the 0 II shell structure. This procedure showed that the 0 II density at $r=3$ cm is $50 \% \pm 30 \%$ of the peak 0 II density, which occurs near $r=8 \mathrm{~cm}$, as is indicated in the figure. This is consistent with dominant beam injection of oxygen, but not with oxygen trapping from low-energy radial sources. Radial oxygen fluxes of 100-eV energies should be reduced more than six orders of magnitude before 
4/17/78 Shots 48-64

Peak O II $B=1.7 \times 10^{15} \mathrm{ph} \mathrm{s}^{-1} \mathrm{~cm}^{-2} \mathrm{sr}^{-1}$

$\int n_{e} d \ell=6.4 \times 10^{14} \exp \left[-y^{2} / 10.7^{2}\right] \mathrm{cm}^{-2}$

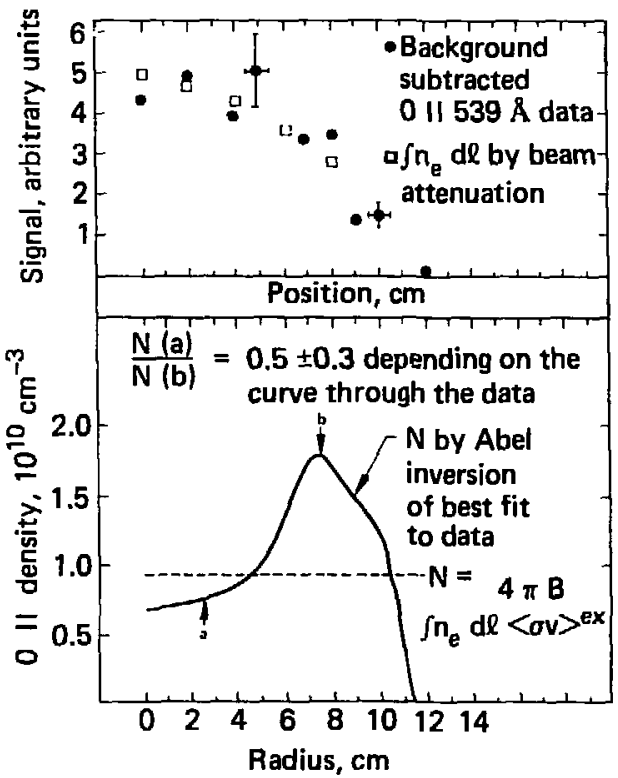

Figure 9. The 0 II $539 \AA$ brightness and the electron line density profiles, are shown above. Below, the variation of density profiles allowed by the data is indicated.

reaching a radius of $3 \mathrm{~cm}$. In contrast, beam injected oxygen can readily penetrate to the center of 2 XIIB; its transmission to a 3 -con radius is $30 \%$. For impurities injected with low energies, only the higher ionization stetes ( 0 V) should exist near the plasma center. The existence of 0 II near the plasma center is good evidence that the neutral oxygen arrives with a high energy •

Low-energy oxygen sources were simulated by injecting neon impurities in two ways. These experiments, which had other implications, are discussed 
further in Section VI and Appendix G. When some neon gas was injected radially by a pulsed valve $55 \mathrm{~cm}$ from the plasma, the Ne IV and Ne V brightnesses were much less than those of oxygen, although the excitation rate coefficients are similar for these two elements. In addition, the plasma was destroyed before enough neon gas could be introduced to obtain a Ne IV brightness comparable to that of $0 \mathrm{IV}$. These facts imply that low-energy radial oxygen sources were normally unimportant.

In the second experiment, a gas-arc plasma gun was used to inject neon ions along field lines. The background-corrected Ne II $460 \AA$ signal peaked before $3.5 \mathrm{~ms}$, then decreased to almost zero by $5 \mathrm{~ms}$. In contrast, 0 II signals from similar plasmas reached a broad plateau during the high-density phase of the discharge, lasting from 4 to 8 ms in this case. The sharp decrease in Ne II emission showed the attenuation of singly ionized axial impurity fluxes as the plasma density increased. The quite different 0 II time behavior indicated that axial oxygen fluxes from the plasma guns were not significant. In addition, al though the streaming plasma was $100 \%$ neon, the Ne IV and Ne V brightnesses were two orders of magnitude below those of 0 IV and $O V$. This also indicates that the streaming plasma is a negligible source of oxygen.

The charge-exchange analyzer provides further evidence that the oxygen is injected by the neutral beams [34]. It is able to resolve the energy components of the neutral beam when the beams are fired into low-pressure neutral gas. A one-tenth energy deuterium component is observed at such times which is consistent with the breakup of $\mathrm{D}_{2} \mathrm{O}$ from the neutral beam [ 35]. Similar results have been obtained at Lawrence Berkeley Laboratory (LBL) and Princeton Plasma Physics Laboratory [ 36,37]. 
In view of the above evidence, it is quite clear that the oxygen in 2XIIB is dominantly beam-injected. In consequence, the oxygen is well confined and radiates substantially, but it also heats the electrons while it is cooling. The ways in which the oxygen affects the power balance of the ions and electrons are considered in section $V-D$. It is found that the oxygen in 2 XIIB causes additional power input to the ions and has 1 ittle net effect on the electrons. This will not always be true in future mirror machines, but it is unlikely that neutral beam injected oxygen will ever be significantly damaging to them; this issue is discussed in section VII.

There is some data to indicate that the neutral beams are not always the only source of oxygen. The 0 II 539 A signal is a good indicator of the neutral oxygen $f 1 u x$, because 0 II ionizes quickly ( $1 \mu s)$. It exhibits two types of time development, although in general only one of them is seen on a given day. The time development which is consistent with negligible lowenergy oxygen fluxes shows a constant plateau brightness until the beams turn off, and then the brightness abruptly declines to almost zero (the hot neutral oxygen is gone in a few microseconds). On other days, the 0 II brightness establishes its plateau value then increases beginning at about $5 \mathrm{~ms}$ until the beams turn off. At that time the signal drops only by the amount that was present at 5 ms and then decays at a rate determined by the plasma decay. The latter behavior indicates the development of a low-energy oxygen flux. Because of its low velocity, this $f l u x$ does not cease immediately when the beams tum off. This observed behavior was at times correlated with an early decay of the plasma density. The highly ionized oxygen states showed no discernible effect of these differences, which is also consistent with the behavior of low-energy impurities (see Section $\nabla I$ ). The question of 
additional low-energy oxygen sources and their effect on the plasma is a possible subject for future research.

C. The Population of Oxygen Ionization States

Once the source of oxygen was understood, the details of its existence in the plasma could be investigated. It was stated in section III that ionization and charge-exchange should be important in 2 XIIB, and may reach equilibrium, although this is subject to the effects of confinement losses. After an examination of oxygen confinement, the population distributions predicted from an equilibrium of ionization and charge-exchange are discussed. These are compared with data obtained over a wide range of plasma parameters, for which the oxygen population distributions varied considerably. The agreement is within the uncertainties involved. One important consequence is that this demonstrates the feasibility of obtaining time-resolved average electron temperature measurements using spectroscopic techniques.

The beam-jnjected, high-energy oxygen is magnetically trapped and is well-confined. The initial oxygen velocity is perpendicular to the magnetic field, and the injection energy ( $16 \mathrm{keV})$ is much larger than the ambipolar hole (which is $2.5 \mathrm{keV}$ for 0 VII with $\mathrm{T}_{e}=120 \mathrm{eV}$ ). In consequence, the oxygen is confined until it loses most of its energy or scatters far enough in velocity space to become unconfined. As may be seen from the lifetimes in Table 1, an oxygen ion should ionize and charge-exchange many times before it is lost. It is confined for a long time compared with the lifetimes of processes which lead to a change of ionization state. 
The effect of the long oxygen-confinement time may be seen by examining the rate equation for the population of $O V I I$. Because of the low electron temperature, ionization from $O$ VII to $O$ VIII is negligible. The resulting low population of 0 VIII insures that charge-exchange into $O$ VII is al so smal1. As was discussed in Section III, chargeexchange is the dominant recombination process. Using the notation of that section, we have:

$$
\frac{d n_{I I}}{d t}=n_{V I} n_{e}<\sigma v>v_{V I}^{i o n}-n_{V I I} n_{D}<\sigma v>c x \text { VII }-\frac{n_{V I I}}{\tau_{V I I}} .
$$

In steady state, if confinement losses are small, this yields

$$
\frac{n_{v I}}{n_{V I I}}=\frac{n_{D}<\sigma v>v I I}{n_{e}<\sigma v>v I}
$$

The rate equation for $O V I$ contains terms due to ionization of $O V$ and $O V I$, and charge-exchange of $O V I$ and $O V I I$. The addition of the rate equations for $O$ VII and $O$ VI eliminates some terms, and gives a relation between $O \mathrm{VI}$ and $\mathrm{O} \mathrm{V}$. This can be continued, to obtain, in general:

$$
\frac{n_{x}}{n_{x+1}}=\frac{n_{D}\left\langle\sigma v>{ }_{x+1}^{c x}\right.}{n_{e}\langle\sigma v\rangle_{x}^{i \text { ion }}}
$$

This is the analogy of the we11-known coronal model [38], applied to a plasma with long confinement and chargeexchange-dominated recombination. The low ionization states have higher populations than such a model indicates, because of the steady influx of new particles.

To compute the average population ratio of two ionization states, we must know $n_{e}$ (see section IV), $n_{D}$, (see Appendix B), $\langle\sigma v\rangle^{c: x}$ (see Section 
III), and $\langle\sigma v\rangle^{i}$. The ionization rate coefficients may be taken from

Lotz [24]; these values are based on simple scalings. Experimental measurements often give cross sections smaller than these values ( 0.6 Lotz) [ 25], hence an additional comparison based on this $f$ act is al so worthwhile.

Comparison of Theory and Experiment

The results of calculations based on $\mathrm{Eq}$. (1) are given in Table 5 . This table shows the predicted fractiona' populations of 0 II through 0 VII, as defined by

$$
P F_{i}=n_{i} / \sum_{j=I I}^{V} n_{j}
$$

The expected relative concentrations of the observed ionization states which is more relevant to the experiment - are al so given. They were obtained as was $P F_{i}$, except that the sum is taken only over the observed states.

Figure 10 and Table 5 compare experimental results with the predictions explained above. The concentrations of oxygen ions were obtained from the observed brightnesses, as described in Appendix c. The metastable populations we taken into account using the metastable ratios shown in Table 5 and discussed in Appendix $c$. The observed factor-of-two agreement between the experimental and predicted results is within the uncertainties of the calculations. In particular, the metastable ratios, the rate coefficients for excitation, ionization, and charge-exchange, and the brightness measurements all carry uncertainties.

The large relative changes in the observed population distributions have two significant consequences. First, they lend further support to the 
TABLE 5. OXYGEN POPULATION DISTRIBUTIONS

\begin{tabular}{|c|c|c|c|c|c|c|c|c|c|}
\hline 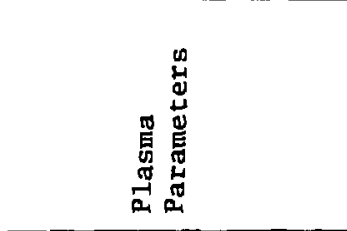 & 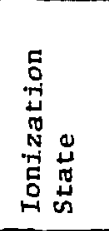 & 尊 & $\overbrace{}^{1}=$ & T's & $(\operatorname{Lotz})$ & 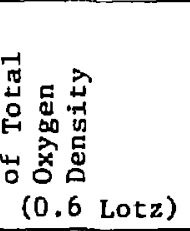 & (Lotz) & 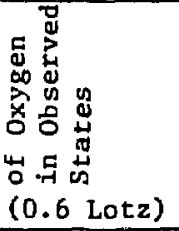 & 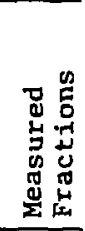 \\
\hline $\mathrm{T}_{\mathrm{e}}=60 \mathrm{eV}$ & $\begin{array}{l}0 \text { VII } \\
0 V I\end{array}$ & 0 & $\begin{array}{l}35 \\
32\end{array}$ & 0.93 & $\begin{array}{l}0.10 \\
0.40\end{array}$ & $\begin{array}{l}0.04 \\
0.27\end{array}$ & 0.44 & 0.28 & 0.21 \\
\hline $\mathrm{n}_{\mathrm{e}}=3.8 \times 10^{13} \mathrm{~cm}^{-3}$ & $\begin{array}{ll}0 & V \\
0 & I V\end{array}$ & $\begin{array}{l}2.1 \\
0.5\end{array}$ & $\begin{array}{l}50 \\
61\end{array}$ & $\begin{array}{l}3.9 \\
16\end{array}$ & $\begin{array}{l}0.36 \\
0.13\end{array}$ & $\begin{array}{l}0.41 \\
0.23\end{array}$ & $\begin{array}{l}0.40 \\
0.14\end{array}$ & $\begin{array}{l}0.43 \\
0.24\end{array}$ & $\begin{array}{l}0.54 \\
0.20\end{array}$ \\
\hline $\mathrm{n}_{0}=4.3 \times 10^{10} \mathrm{~cm}^{-3}$ & $\begin{array}{ll}0 & \text { III } \\
0 & \text { II }\end{array}$ & 1.5 & 2.2 & $\begin{array}{l}50 \\
165\end{array}$ & $\begin{array}{c}0.02 \\
0\end{array}$ & $\begin{array}{c}0.05 \\
0\end{array}$ & $\begin{array}{c}0.02 \\
0\end{array}$ & $\begin{array}{c}0.05 \\
0\end{array}$ & $\begin{array}{l}0.04 \\
0.01\end{array}$ \\
\hline $\mathrm{T}_{\mathrm{e}}=120 \mathrm{eV}$ & $\begin{array}{l}0 \text { VII } \\
0 \text { VI }\end{array}$ & & & 3.4 & $\begin{array}{l}0.48 \\
0.41\end{array}$ & $\begin{array}{l}0.33 \\
0.46\end{array}$ & 0.80 & 0.69 & 0.54 \\
\hline$n_{e}=4.9 \times 10^{13} \mathrm{~cm}^{-3}$ & $\begin{array}{ll}0 & V \\
0 & I V\end{array}$ & & & $\begin{array}{l}11 \\
36\end{array}$ & $\begin{array}{l}0.09 \\
0.01\end{array}$ & $\begin{array}{l}0.18 \\
0.03\end{array}$ & $\begin{array}{l}0.18 \\
0.02\end{array}$ & $\begin{array}{l}0.27 \\
0.04\end{array}$ & $\begin{array}{l}0.39 \\
0.04\end{array}$ \\
\hline$n_{0}=4.0 \times 10^{10} \mathrm{~cm}^{-3}$ & $\begin{array}{ll}0 & I I I \\
0 & I I\end{array}$ & & & $\begin{array}{l}92 \\
260\end{array}$ & $\begin{array}{l}0 \\
0\end{array}$ & $\begin{array}{l}0 \\
0\end{array}$ & $\begin{array}{l}0 \\
0\end{array}$ & $\begin{array}{l}0 \\
0\end{array}$ & $\begin{array}{l}0.02 \\
0.01\end{array}$ \\
\hline $\begin{array}{l}\mathrm{T}_{\mathrm{e}}=40 \mathrm{eV} \\
\mathrm{n}_{\mathrm{e}}=2 \times 10^{13} \mathrm{~cm}^{-3}\end{array}$ & $\begin{array}{ll}0 & V I I \\
0 & V I \\
0 & V \\
0 & I V\end{array}$ & & & $\begin{array}{l}0.26 \\
1.32 \\
7.4\end{array}$ & $\begin{array}{l}0.003 \\
0.071 \\
0.33 \\
0.42\end{array}$ & $\begin{array}{l}0.03 \\
0.20 \\
0.46\end{array}$ & $\begin{array}{l}0.18 \\
0.82\end{array}$ & $\begin{array}{l}0.13 \\
0.87\end{array}$ & $\begin{array}{l}0.17 \\
0.77\end{array}$ \\
\hline $\mathrm{n}_{0}=3.8 \times 10^{10} \mathrm{~cm}^{-3}$ & $\begin{array}{ll}0 & I I I \\
0 & I I\end{array}$ & & & $\begin{array}{l}28 \\
109\end{array}$ & $\begin{array}{c}0.18 \\
0\end{array}$ & $\begin{array}{c}0.31 \\
0\end{array}$ & 0 & 0 & 0.06 \\
\hline
\end{tabular}




$$
\begin{aligned}
& \overline{\mathrm{T}}_{\mathrm{e}}=120 \mathrm{eV} \\
& \overline{\mathrm{n}}_{\mathrm{e}}=4.9 \times 10^{13} \mathrm{~cm}^{-3} \\
& \bar{n}_{\mathrm{D}}=4.0 \times 10^{10} \mathrm{~cm}^{-3}
\end{aligned}
$$

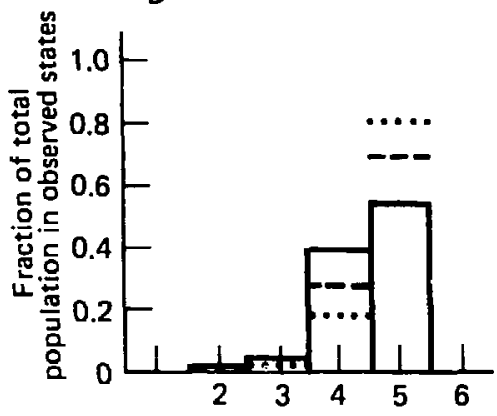

$\overline{\mathrm{T}}_{\mathrm{e}}=60 \mathrm{eV}$

$\bar{n}_{e}=3.8 \times 10^{13} \mathrm{~cm}^{-3}$

$\overline{\mathrm{n}}_{\mathrm{D}}=4.3 \times 10^{10} \mathrm{~cm}^{-3}$

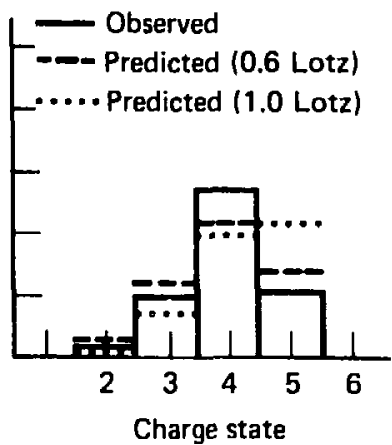

$\overline{\mathrm{T}}_{\mathrm{e}}=40 \mathrm{eV}$

$\overline{\mathrm{n}}_{\mathrm{e}}=2.0 \times 10^{13} \mathrm{~cm}^{-3}$

$\overline{\mathrm{n}}_{\mathrm{D}}=3.8 \times 10^{10} \mathrm{~cm}^{-3}$

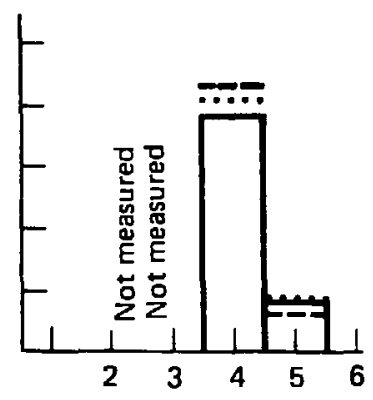

Figure 10. Observed and predicted population distributions of oxygen among the observed ionization states. The observed distributions (solid lines) vary considerably as plasma conditions change. The predictions agree adequately with the data. The use of ionization rate coefficients equal to 0.6 times the tabulated values (Lotz[24]) gives better agreement than the full values do.

account of oxygen given above, and to theconclusions of Section III about the important atomic processes in this plasma. Second, they show the possibility of a useful diagnostic. Given beam current, line density, and plasma radius, which determine the electron and neutral densities, the model of Eq. (1) can be applied to any 2 XIIB plasma. If simul taneous measurements of $0 \mathrm{IV}, 0 \mathrm{~V}$, and $O$ VI emissions are made, the magnitude and time variation of average electron temperature can be determined. The metastable ratios and other numerical factors could be "calibratea" by comparison with single point Thomson scattering measurements. This hypothetical diagnostic could be applied to 2XIIB and future mirror machines. 
The oxygen in 2XIIB increases the power input to the deuterons by about $15 \%$ and causes much less net power loss fram the electrons. This is surprising because the oxygen concentration is only $3 \%$. Our accepted notion that impurities are harmful leaves us even more surprised. The effects of oxygen on the deuterons and on the electrons are evaluated. Because of the small oxygen concentration, the power carried by the oxygen ions is ignored. The dominant mechanism by which oxygen adds power to the ions is the trapping of neutral deuterium. The di rected neutral beam current is attenuated by ionization and charge-exchange. When deuterons trap beam neutrals by charge-exchange (which happens to about $75 \%$ of the attenuated neutrals), a high-energy deuterium neutral is produced; it most often escapes from the 2XIIB plasma. In contrast, the oxygen ions are not lost when they trap beam neutrals by charge-exchange, because they are multiply ionized. This is significant in 2XIIB, but would not be important in a larger plasma from which charge-exchange neutral products did not normally escape.

Calculations of Oxygen Effects

The effect of oxygen on the plasma power balance is considered in two ways below. First, the effects of oxygen in the plasma are evaluated by computing the power gained or lost per mit volume by several processes. The results of these calculations are shown in Table 6. Second, the attenuation of the neutral beams in the presence of oxygen is considered, and the incremental power input to the ions is determined. In both cases, the oxygen is found to enhance the power input to the deuterons by about $15 \%$. 
TABLE 6. THE EFFECTS OF OXYGEN ON THE 2XIIB POWER BALANCE

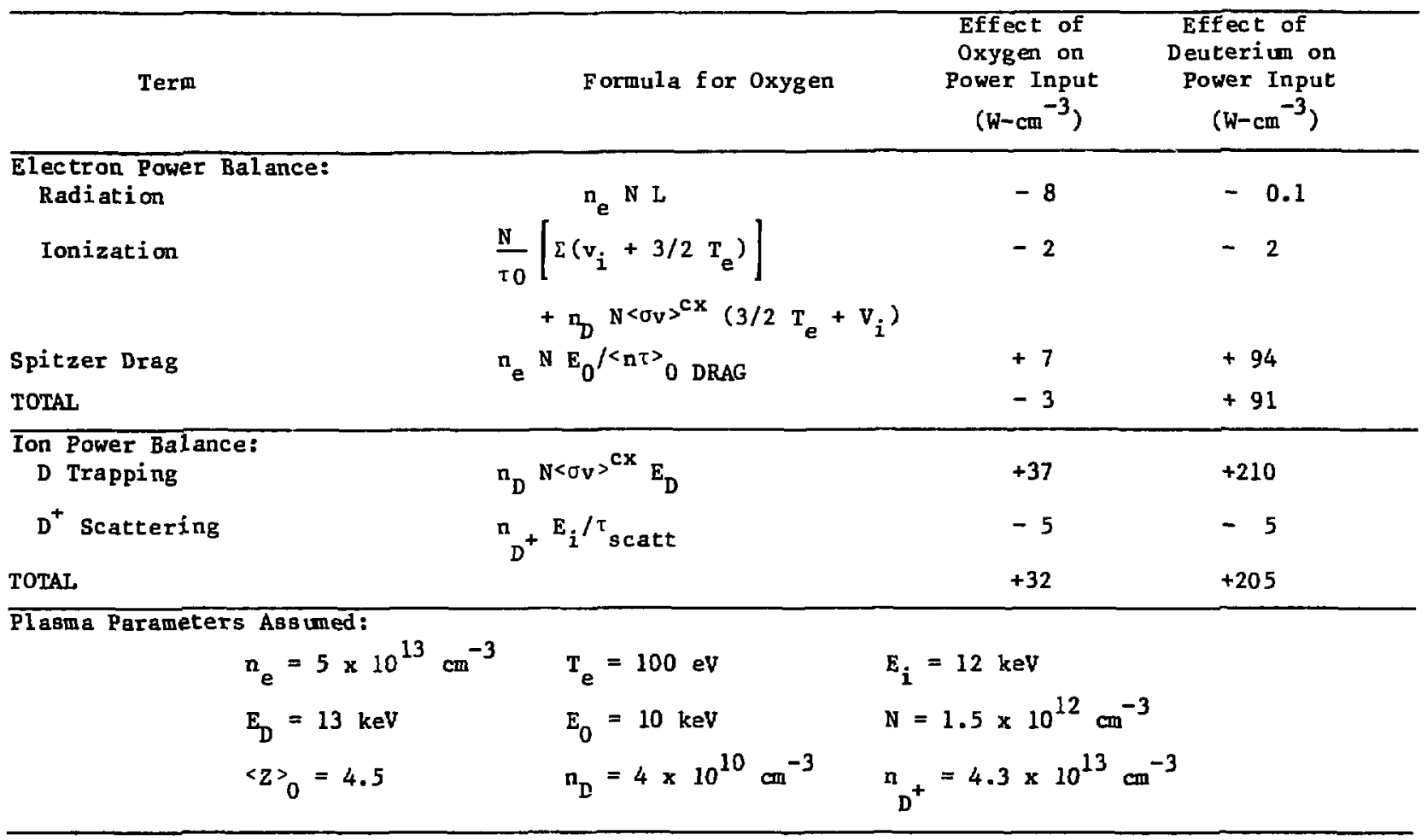


Table 6 sumarizes the effects of oxygen on the electrons and on the deuterons. Comparative results for the deuterium ions are also given. To calculate the effects considered, typical 2 XIIB parameters were used; these are given in the table. The processes listed are now considered individually, and some technical coments are made.

The radiation power $108 \mathrm{~s}$ was computed by using the cooling rate, $L=10^{-25} \mathrm{~W}-\mathrm{cm}^{3}$. The value of $\mathrm{L}$ was determined by comparison with the results presented in Appendix $C$. The deuterium neutrals radiate about $0.1 \mathrm{H}^{\mathrm{H}} \mathrm{cm}^{-3}$ of Lyman alpha emissions.

The ionization power loss results $f$ rom the liberation of cold electrons within the plasma. It is the oxygen current per unit volme $\left(\mathrm{N} / \tau_{0}\right)$ times the energy loss involved in the initial ionization of oxygen u to 0 VII, $\Sigma\left(3 \mathrm{~T}_{\mathrm{e}} / 2+\mathrm{v}_{\mathrm{i}}\right)$, added to the electron power loss resulting when oxygen ions are re-ionized after undergoing charge-exchange with neutral beam atoms. The neutral-beam atoms that are ionized within the plasma produce cold electrons, leading to roughly a $2 \mathrm{~W}^{-\mathrm{cm}^{-3}}$ power drain on the electrons in the plasma.

The oxygen and the deuterons heat the electrons by Spitzer drag, which was evaluated using by the formula given in Table 2. This is the primary mechanism by which electrons are heated in 2XIIB. It is seen that the electron heating by the oxygen ions nearly offsets the oxygen-induced power losses, so that the net effect of the oxygen on the electrons is a very small power loss--about $3 \%$ of the electron heating by the deuterium ions.

The deuteron power balance is affected by the oxygen in two ways. The oxygen traps neutral deuterium, as noted above, and it scatters confined deuterons into the loss cone. The power input due to the trapping of neutral deuterium by charge-exchange with oxygen ions is the trapped current per unit 
volume ( $n_{D} N<\sigma v>^{c x}$ ) times the neutral deuterium energy $\left(E_{D}\right)$. For comparison, the effect of deuterium on the power input by D trapping includes the power input resulting from the ionization of deuterium and the chargeexchange of deuterium on deuterons.

The power loss due to scattering into the loss cone is the loss current per unit volume due to scattering ( ${ }_{D^{+}} / \tau_{\text {Bcat }}$ ) times the mean ion energy $\left(E_{i}\right)$. The lifetime $T_{\text {scat }}$ was computed using the resul ts of Futch et al. [ 30] and spitzer [29]. The power loss due to this mechanism is seen to be small for both oxygen and deuterons. These values are comparable because the high charge of the oxygen offsets the difference in density.

The 3\% oxygen concentration in 2 XIIB results in a small electron power loss, and increases the power input to the deuterium by about $15 \%$, on the basis of the above calculations. The effect of oxygen on the deuteron power balance can al so be evaluated by considering the attenuation of the neutral beams .

The power input to the ions from the neutral beams is evaluated by computing the transmission of the neutral beams and then finding the power deposited by the various attenuating processes. This procedure is followed below, assuming a small impurity concentration. The volume-averaged beam transmission is

$$
T=\left[\exp -\int n_{e} d l \sigma_{e f f} / 1.41\right],
$$

in which $n_{\mathrm{e}} \mathrm{dl}$ is the peak $1 \mathrm{ine}$ density and the factor 1.41 results from volume averaging (it was determined by comparison with a computer code that celculates beam attenuation [5]). In the presence of an impurity,

$$
\begin{aligned}
\sigma_{\text {eff }}=\frac{1}{v}\left(1-\phi<Z \geqslant\left(\langle\sigma v\rangle_{D^{+}}^{\text {ion }}+\langle\sigma v\rangle_{D^{+}}^{\mathrm{cx}}\right)\right. \\
+\langle\sigma v\rangle_{\mathrm{en}}^{\text {ion }}+\alpha\langle\sigma v\rangle_{\text {imp }}^{\mathrm{cx}}
\end{aligned}
$$


in which $\phi$ is the impurity concentration relative :o electron density, $\langle Z\rangle$ is its average charge, and $v$ is the average beam neutral velocity. The rate coefficients are for charge-exchange on deuterons $\left(\langle\sigma \gamma\rangle_{D}^{c x}\right)$, ionization by deuterons and electrons $\left(<\sigma_{v}>_{D}^{i o n}\right.$ and $<\sigma v_{e}^{j}$ in $)$ and charge-exchange on impurities such as oxygen $\left(\left\langle\sigma_{v}\right\rangle_{\text {imp }}^{c x}\right.$ ). The change in $\sigma_{\text {eff }}$ due to the impurity is

$$
\Delta \sigma_{\text {eff }}=-\frac{\alpha}{v}\left[\langle z\rangle\left(\left\langle\sigma_{v}\right\rangle^{c x}+\langle\sigma v\rangle_{D^{+}}^{i o n}\right)-\langle\sigma v\rangle_{\text {imp }}^{c x}\right] .
$$

The decrease in beam attenuation due to the relative absence of deuterium ions is partially offset by charge-exchange on impurity ions. The net change is small because $\alpha$ is small. On $2 X I I B, \sigma$ eff decreases roughly $5 \%$. ( $\sigma_{\text {eff }}=1.54 \times 10^{-15}$ without oxygen, and is $1.47 \times 10^{-15}$ with $3 \%$ oxygen.) In consequence, the beam-attenuation-line-density diagnostic is not significantly afiected by the oxygen in 2 XIIB.

The power input to the deuterons is calculated from the beam trapping and includes the effects of several processes. The power input is

$$
P=I_{b}(I-T) E_{\text {trap }} E_{b}+\left(1-E_{\text {trap }}\right)\left(E_{b}-E_{i}\right)
$$

The attenuated beam current $\left(I_{b}(1-T)\right)$ is partially trapped without the loss of a high-energy deuteron, leading to an energy input ( $\left.f_{t r a p} E_{b}\right)$. The fraction of the attenuated beam trapped in this manner is $f_{\text {trap. The }}$ average beam energy is $E_{b}$. The remaining fraction of the attenuated beam ( 1 - $f_{\text {trap }}$ ) deposits only the difference between the beam energy and the deuteron energy, $\left(E_{b}-E_{i}\right)$. into the plasma. Neglecting reionization and trapping of deuterium charge-exchange neutral products, we can estimate $f_{\text {trap }}$ In this approximaticn

$$
f_{\text {trap }}=\frac{\langle\sigma v\rangle^{i} D^{+}(1-\alpha\langle z\rangle)+\langle\sigma v\rangle^{i o n}+\langle\sigma v\rangle_{\text {imp }}^{c x}}{v e_{\text {eff }}}
$$


This equation computes the fraction of the total effective cross-section for neutral beam attenuation which is due to processes other than charge-exchange with plasma deuterons. The increment in this fraction due to impurities is

$$
\Delta \mathrm{f}_{\text {trap }}=\frac{\phi}{\mathrm{v} \sigma_{\text {eff }}}\left(\langle\sigma \mathrm{v}\rangle_{\text {imp }}^{c x}-\langle\mathrm{Z}\rangle\langle\sigma \mathrm{v}\rangle_{\mathrm{D}^{+}}^{\text {ion }}\right) \simeq 0.05 .
$$

The resulting additional power input is

$$
\Delta P=I_{b}(1-T) \Delta f_{\text {trap }} E_{b}=220 \mathrm{~kW},
$$

which is $\sim 15 \%$ of the total deposited power (using the plasma parameters of Oc tober 24,1977$)$.

The implications of the effects of oxygen on the power input may be considered. Recent 2 XIIB results are changed little by this discovery. This increased power input implies a $15 \%$ reduction in the energy confinement time, compared to the result obtained without considering the oxygen. This result is within the uncertainty of previous measurements. The increase in power input per unit volume applies to both small and large plasmas, and in consequence does not change the essential results of the plasma radius scaling experiments. In general, while a $15 \%$ effect is significant, it is not substantial enough to alter major experimental results on this type of device. 


\section{A. Introduction}

The plasma potential should prevent the penetration of impurities along field lines, and should quickly eject any low energy gas which strikes the plasma. These effects were not apparent in the initial EUV study of 2XIIB. To investigate the effect of the plasma potential on impurities in 2XIIB, two neon-injection experiments were done. In the first, impurity penetration was studied when neon plasma, streaming along field lines, was used to stabilize 2XIIB. In the second, the penetration and transport of low-energy neon gas was examined by puffing neon gas radially across field lines toward the plasma. In both experiments, the expected effects were observed. Most of the injected impurities did not penetrate or were poorly confined. These results are the subject of this section. Appendix G contains a detailed description of the conduct of these experiments, along with a compilation of some of the data obtained.

\section{B. Summary of Neon Streaming Plasma Penetration}

The injection of neon streaming plasma showed that the penetration of low-energy particles along field lines is impeded wher the high-density, beam-fueled plasma is present. The streaming plasma required to maintain the hot plasma was supplied by gas arc plasma guns operated with neon gas. When these gas guns were fired through the magnetic mirror, a low-energy and low-density plasma was produced. Standard plasma diagnostics found $\mathrm{T}_{\mathrm{e}} \sim 5 \mathrm{eV}, \mathrm{n}_{\mathrm{e}} \approx 1.5 \times 10^{13} \mathrm{~cm}^{-3}$, and $\mathrm{E}_{\mathrm{i}} \lesssim 250 \mathrm{eV}$. Because of the low electron temperature, this plasma was assumed to be entirely Ne II. 
When the beam-fueled plasma was present, the amount of neon reaching the midplane was determined spectroscopica11y. The observed time development, brightnesses, spatial profiles of neon emissions, and the neon density are discussed in Appendix $G$. The amount of neon which reached the midplane was a small fraction ( $(4 \%)$ of the previous value. This is consistent with the expected rejection of most of the neon by the plasma potential. The characteristics of this plasma, stabilized using neon streaming plasma, are compared with normal deuteriun-stabilized plasmas and with theoretical expectations in Appendix $G$.

\section{Description and Summary of Radial Puffing}

In this section, the radial neon-puffing experiment is summarized. First, the operation of 2XIIB for this experiment is described; and then the absorbtion of the neutral neon flux at the plasma boundary is evaluated. The data are summarized and analyzed in later sections. The analysis and several of the measurements, re not accurate to better than a factor of 2. However, the conclusions drawn from this experiment are not affected by factor-of-2 variations .

A pulsed valve near the midplane, located $55 \mathrm{~cm}$ from the plasma center, was used to puff a cloud of neon gas toward the plasma. The resulting neutral neon density was measured with a fast ionization gauge when no plasma was present. The extent of the gas cloud was measured to be large compared to the plasma dimensions. Duxing the experiments, the neon gas was introduced after the buildup of the high-density plasma. The neon flux could be varied and if it was high enough the plasma was destroyed. The data analyzed below was obtained with low neon fluxes; the plasma was either in steady state or was slowly decaying (with a 2-ms decay time). 
As described in section III, interpretation of the observed brightnesses requires knowledge of the electron density, the emitting region, and the electron temperature. The neon flux was ass uned to have been attenuated in a shell located near the edge of the plasma, where the electron density is about $1.5 \times 10^{13} \mathrm{~cm}^{-3}( \pm 30 \%)$. Spatial scans of neon emissions showed profiles which were consistent with the absorbtion of most of the neon near the plasma boundary $\left(r \sim R_{p}=9.3 \mathrm{~cm}\right)$; also, negligible emissions were observed at radial positions beyond $10.5 \mathrm{~cm}$. The neutral neon thus appears to have been ionized at radii near the plasma sadius, $R_{p}$, where the electron density was inferred from beam attenution measurements to have been near $1.5 \times 10^{13} \mathrm{~cm}^{-3}$. This is expected, because a $1 / 40-\mathrm{eV}$ neutral neon flux is attenuated more than $60 \%$ per centimeter if the electron density is $10^{13} \mathrm{~cm}^{-3}$ (and $\mathrm{T}_{\mathrm{e}} \geqslant 30 \mathrm{eV}$ or $\mathrm{E}_{\mathrm{i}} \sim 10 \mathrm{keV}$ ). The uncertainty in the electron density is due to uncertainty of the exact profiles of electron density and neon ionization. The shell width $\Delta w$ is unimportant because it is not needed to compute the results quoted below.

To analyze the neon data, it was assumed that the electron temperature was about $50 \mathrm{eV}$ in the emitting region. The electron temperature has been observed to vary little as radius increases (see section C3); it was normally 60 to $70 \mathrm{eV}$ near the center of plasmas similar to those observed here. In addition, the Ne V $571 \AA$ and Ne III $490 \AA$ lines had similar brightnesses and began to emit at nearly the same time. This indicates that Ne IV was being ionized quickly by comparison with the time response of the spectrometer system, which requires $T_{e} \succsim 50 \mathrm{eV} . \quad T_{e}$ as 1 ow as $30 \mathrm{eV}$ was not allowed by these data.) 
As is shown in this section, the measured neon brightnesses are consistent with the diversion of neon ions awav - the 2XIIB midplane by the plasma potential, but rot with loss mechanisms that confine the neon for longer times. The observed brightnesses can be related to the neon loss mechanism, because the rate at which neon was deposited in the plasma is known from the neutral density measurements. If the neon was diverted by the plasma potential, so that the neon ions always flowed away from the midplane, only those ions deposited near the midplane would have been observed by the monochromator. In contrast, if the neon could flow out of the machine in either direction, the neon density at the midplane would have been much higher for the same flux of neon, since ions deposited along the entire plasma would contrioute to it. Even longer neon confinement would have led to larger brightnesses.

The observed brightnesses are tabulated in Table 7; they have uncertainties of $+50 \%$. The sources of uncertainty include the calibration, the centering of the emission line on the spectrometer slit, and the background subtraction, which was based on the signal present before the neon was introduced. Ne VI $402 \AA$ emissions were less bright than Ne V $571 \AA$ emissions, and the $160-e V$ ionization potential of Ne VI implies that little Ne VII was produced. The excitation rate coefficients in the table were most graciously provided by Dr. J. Davis of NRL 39 . The neon line density at the midplane $\left(\Delta_{\mathrm{WN}}\right)$ was estimated using Eq. (III-10) for only one shell:

$$
\Delta w N=\sum_{i} \Delta w N_{i}=\sum_{i} \frac{4 \pi B_{i}}{n_{e}<\sigma v>c x}
$$


TABLE 7. BRIGHTNESSES AND FLUXES DURING NEON GAS PUFFING

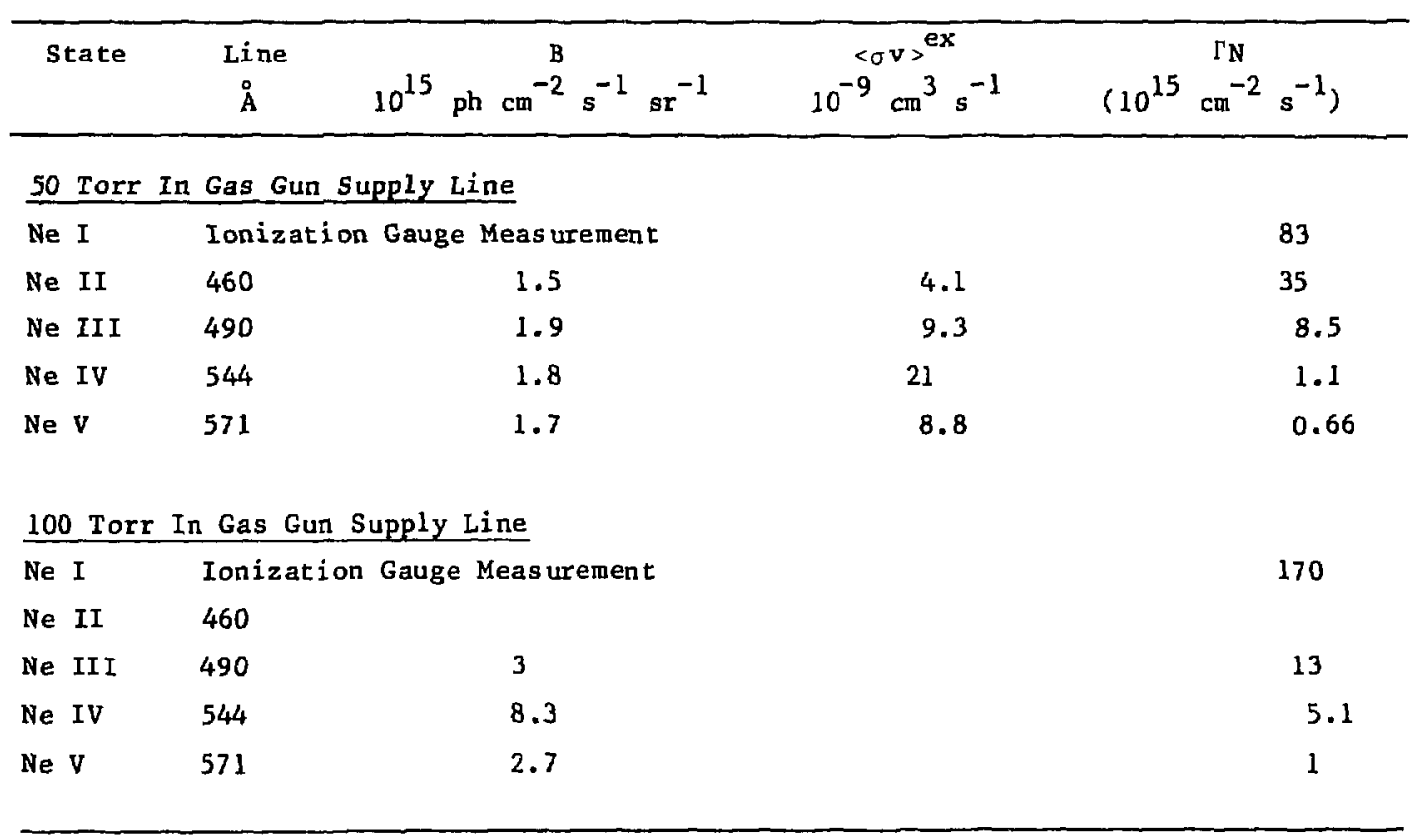


The sum was taken over all observed neon states, and the result was that $\Delta w N=7 \times 10^{11} \mathrm{~cm}^{-2}$. The total neon line density is probably about twice this value, because the metastable states and Ne VI were not observed.

The neon line density can be related to the neutral neon flux and the neon confinement time. If the neon is diverted away from the midplane of 2XIIB, the neon line density $(\Delta w N)$ at the midplane is the neutral neon flux times the average time a neon ion remains in the fiold of view of the spectrometer. That is,

$$
\Delta w \mathbf{N}=\Gamma_{0} \ell / v,
$$

in which $v$ is the neon velocity. The potential has only a small effect on the neon velocity near the midplane, $v \stackrel{n}{=} 10^{5} \mathrm{~cm} s^{-1}$. The average distance an ion travels in the field of view of the monochromator is $\varepsilon(1.5 \mathrm{~cm})$. The neutral flux, $\Gamma_{0}$, was measured to be $8.3 \times 10^{16} \mathrm{~cm}^{-2} \mathrm{~s}^{-1}$ by using an ionization gauge. Hence we predict wN $=1.3 \times 10^{12} \mathrm{~cm}^{-2}$, if the neon is diverted by the plasma potential. This agrees with the value indicated by the meas urements .

By contrast, the measured neon line density is $f$ ar below the value which would result if all the neon ions could flow out either end of the machine. In this case half the neon current striking the plasma would flow through the midplane in a semicircular shell. The neon density at the midplane would be half the neon current divided by the neon velocity divided by the area of the surface through which it flows:

$$
N=\frac{I_{N e}}{2} \frac{l}{v} \frac{I}{\pi R_{P} \Delta w}
$$


The neon current is the neutral flux times the cross-sectional area of the plasma:

$$
I_{N e}=r_{0} 2 R_{P} L
$$

The length of the plasma is $L(40 \mathrm{~cm})$. Thus

$$
\Delta w_{N}=\Gamma_{0} L /(\pi v)
$$

and we predict $\Delta w N=1.1 \times 10^{13}$. This prediction is eight times larger than the maximun value expected from the data (assuming the population of metastable states is equal to the ground state population). This is inconsistent with the data, even considering the uncertainties in data and analysis. We conclude that the neon deposited away from the midplane is diverted and never flows through it.

\section{E. Neon Fluxes and Neon Confinement}

A similar conclusion may be reached by a different analysis. It is possible to estimate the neon fluxes and to evaluate the neon confinement using the data. This is done below, and these estimates show that the neon ions quickly leave the midplane region, which is consistent with the existence of an ejecting potential.

The inward flux of impurities deposited on the surface of a plasma is related to the rate at which they ionize into successively higher states. This relation is derived by considering the ionization rate equations. The rate equation for the neutral species is:

$$
\frac{\mathrm{dn}}{\mathrm{d} t}=s-n_{e}<\sigma_{v}>{ }_{I}^{i o n} n_{I}+n_{D}<\sigma_{v}>{ }_{I I}^{c x} n_{I I},
$$


in which $n_{D}$ is the neutral deuterium density, and $s$ is the volume rate of deposition. $\left(\Gamma_{0}=s \Delta_{w}\right.$ is the neutral Elux.) For ionized states:

$$
\begin{aligned}
& \frac{d n_{i}}{d t}=n_{e}<\sigma_{v}>i o n \\
& i-1 n_{i-1}-n_{D}<\sigma v>\frac{c x}{i} n_{i} \\
&-n_{e}<\sigma v>_{i}^{i o n} n_{i}+n_{D}<\sigma v>{ }_{i+1}^{c x} n_{i+1}-\frac{n_{i}}{\tau_{i}},
\end{aligned}
$$

where $\tau_{i}$ is the confinement time of state $i$. In steady state, the rate equations for several states can be summed to yield

$$
\sum_{i=1}^{N} \frac{d n_{i}}{d t}=s-\sum_{i=2}^{N} \frac{n_{i}}{\tau_{i}}-n_{e}<\sigma>>_{N}^{i o n}+n_{D}<\sigma v<{ }_{N+1}^{c x} n_{N+1}=0
$$

Equation (7) can be integrated along a line of sight that passes through one shell of width $\Delta w$, to obtain

$$
n_{e}<0 v>_{N}^{i \text { on }} \Delta w n_{N}-n_{D}<0 v>_{N+1}^{c x} \Delta w n_{N+1}=\Gamma_{0}-\sum \frac{\Delta w n_{i}}{\tau_{i}} \cdot \text { (8) }
$$

Now, realizing that

$$
\Delta w n_{N}=\frac{4 \pi B_{N}}{n_{e}<\sigma v>_{N}^{e x}}
$$

from Eq. (III-10), we find

$$
\frac{4 \pi B_{N}\langle\sigma v\rangle_{N}^{\text {ion }}}{\langle\sigma v\rangle_{N}^{e x}}-\frac{\left.4 \pi B_{N+1}<\sigma v\right\rangle_{N+1}^{c x} n_{D}}{\left\langle\sigma v \sum_{N+1}^{e x} n_{e}\right.}=\Gamma_{0}-\sum_{i=2}^{N} \frac{\Delta w n_{i}}{\tau}
$$

The right side of Eq. (10) expresses the inward impurity flux in ionization state $N$ as the neutral flux minus the decrease in $f l u x$ due to the confinement Iosses out of the appropriate states. On the left, this flux is expressed as the quantity $\Gamma_{N}$, defined by 


$$
\Gamma_{N} \equiv 4 \pi B_{N}<\sigma v>{ }_{N}^{i o n} /<\sigma v>{ }_{N}^{e x}
$$

minus a correction for recombination processes. The correction is usually sma11. (It is at most a $30 \%$ effect for this experiment.) Thus, Eq. (10) expresses the important result of this analysis.

Three further comments may be made regarding the implications of Eq. (10). First, if recombination processes (such as charge-exchange) can be neglected, and if confinement times are long compared to ionization times, then $\Gamma_{N}=\Gamma_{0^{\circ}}$. This well-known formula [33] calculates the particle flux as the photon flux (4IIB) times the average number of ionizations per photon $\left(<\sigma v>_{N}^{i o n} /<\sigma v>{ }_{N}^{c x}\right)$. Second, substantial drops in $\Gamma_{N}$ from one state to the next indicate confinement loss rates comparable to or greater than the ionization rate. Third, the confinement times $\tau_{i}$ may in principle be computed starting with the lowest ionized state. In this experiment, the uncertainties are too large to use this technique.

The "fluxes" $\Gamma_{N}$ for the observed neon states are shown in Fig. 11 and were tabulated in Table 7 . The quantity $\Gamma_{N}$ decreases very rapidly as ionization state increases, indicating rapid loss of the neon ions. Decreases of $\Gamma_{\mathrm{N}}$ as charge state increased, like those discussed here, were observed on two other days.

A very crude model of neon confinement can be compared with this data. If we assume neutral neon to be confined for a single fixed time $t$, then the fractional fluxes $\left(\Gamma_{\mathrm{N}} / \Gamma_{0}\right)$ can be computed. Figure 12 shows predicted fractional fluxes for fixed confinement times of $7 \mu \mathrm{s}$ and $100 \mu \mathrm{s}$. The

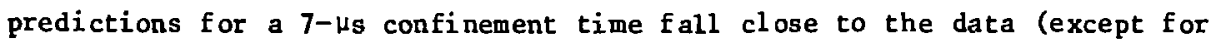
Ne v). The scaling of the 100- $\mu$ s results is, in contrast, quite different from the scaling of the data. This indicates that most of the neon observed at the midplane has not been confined $100_{\mu} \mathrm{s}$. 

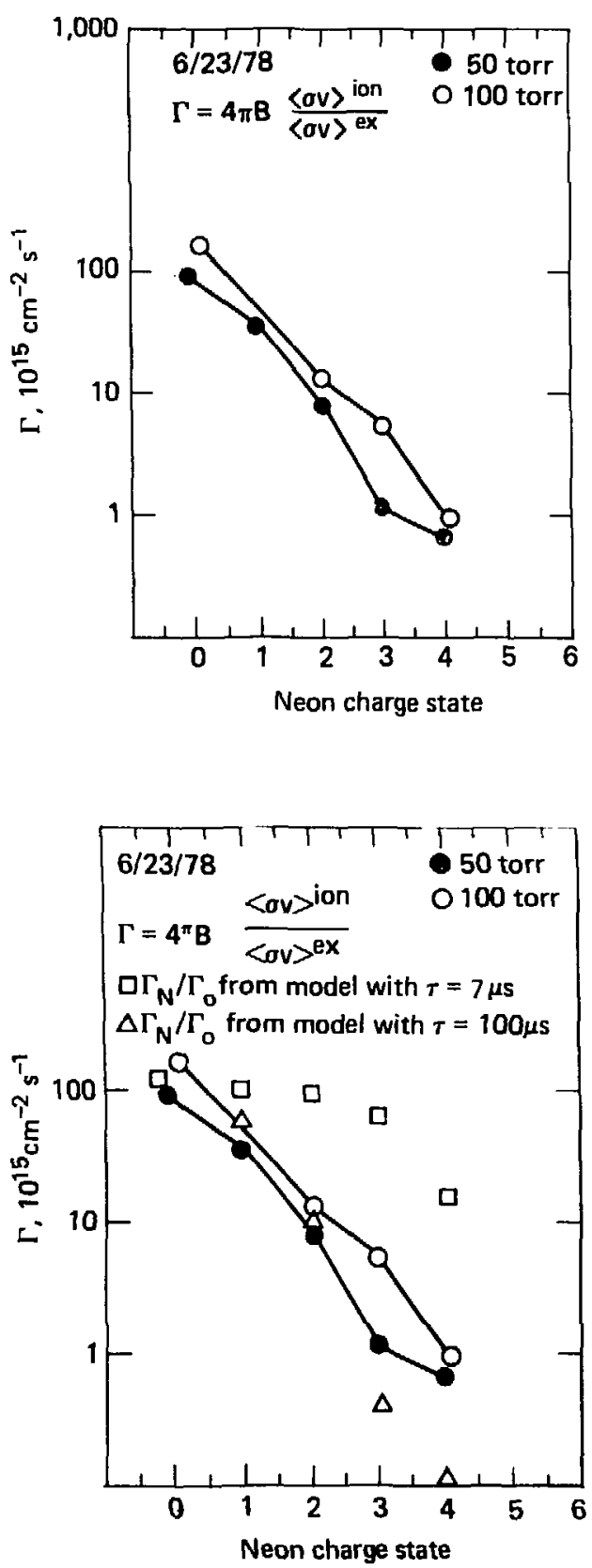

Figure 11. The observed neon fluxes show a sharp decline as charge state increases, for two different gas-valve-supply pressures.
Figure 12. The results of a simple model suggest that the observed neon was confined for much less than $100 \mu s$. 
In sumary, on the basis of the neon brightnesses and neon fluxes, the neon gas injected radially into 2 XIIB is diverted by the plasma potential and soon leaves the central plasma. In this way, the argument that mirror machines should eject impurities has been substantiated, for the low energy impurities which were originally considered.

In closing, it should be noted that these results only apply to most of the neon. In both the radial and the axial experiments, different results are obtained for a few percent of the neon. For example, in the radial puffing experiment some neon is observed on the side of the plasma opposite the gas valve. In addition, there is more $\mathrm{Ne} V$ and $N e \mathrm{VI}$ in the plasma than we might expect. Further study of these exceptions may provide better understanding of the carbon and titanium transport and confinement in $2 X I I B$. 


\section{CONCLUSIONS}

This study has shown that an EUV diagnosis of a mirror machine does provide significant new insight; in particular, it has shown that a mirror confined plasma can contain impurities. The first section of this section reviews this study, to highlight its important results. Our knowledge of impurities in mirror machines, and of EUV emissions from them, has been substantially increased. In consequence it is appropriate to examine the future in two respects. It is worthwhile to examine how the basic techniques of plasma spectroscopy developed by Hinnov, Griem, and others, and the instrumentation now being developed at Johns Hopkins can be applied to mirror machines in the future. In addition, the likelihood and effects of impurities in future mirror plasmas can be assessed with more confidence than was possible before. The second section of this section considers the effects of .impurities in mirror reactors from a general standpoint. The third section addresses the problem of impurities and the uses of EUV spectroscopy in the Tandem Mirror Experiment (TMX). Finally, possible EUV diagnostics for the Mirror Fusion Test Facility (MFTF) are discussed in the final section.

\section{A. Summary of These Results}

The EUV emissions from the central plasma in $2 X I I B$ were measured using a normal-incidence spectrophotometer. Emission lines of oxygen, titanium, carbon, and nitrogen were identified on the basis of shot to shot spectral scans. The observed brightnesses were quite 1 arge by comparison with those reported from other fusion research plasmas. Oxygen was the dominant impurity; the O V $630 \AA$ and O VI $1032 \AA$ emission brightnesses were often 1 arger than $10^{18} \mathrm{ph} \mathrm{s}^{-1} \mathrm{~cm}^{-2} \mathrm{sr}^{-1}$. The oxygen concentration was a few percent in 
the 2 XIIB plasma, in spite of the plasma potential, because the oxygen was injected by the neutral beams. The oxygen is very energetic ( $10 \mathrm{kev})$, and the spatial profile of 0 II $539 \AA$ emissions shows that neutral oxygen penetrates deep into the plasma. The $O$ II emissions also depend on beam current in a manner consistent with beam injection of the oxygen. Neon was injected into 2XIIB, either as gas puffed radially or as plasma streaming along field lines, and the observed neon emissions behaved quite differently from those of oxygen. The oxygen is injected with a large velocity perpendicular to the magnetic field, so it is well-confires by the magnetic mirrors. In consequence, the small fraction of oxygen in the neutral beams results in a small fraction of oxygen in the plasma.

Because of its high energy, the oxygen is not substantially affected by the plasma potential. The potential should act to reduce the concentrations of most impurities. The neon injection experiments substantiated this hypothesis. The neon streaming plasma was kept from penetrating, and the radial neon flux was diverted and ejected. The 2 XIIB mirror machine thus worked as expected: low-energy impurities were poorly confined.

However, some other mechanisms that affect the impurities in 2 XIIB are not understood. When the neon gas was puffed radially, a small fraction of the neon ions were apparently confined a long time. The carbon and titanium in $2 X I I B$ are not injected by the neutral beams, and are not injected by the streaming plasma guns. Yet these impurities show steady-state concentrations of a few tenths of a percent, and the carbon energy is several keV. These effects are possible topics for future research. Interesting physical mechanisms may be involved, and the possible impurity problems in a reactor cannot be fully assessed until these mechanisms are understood. 
Impurities have at least three significant harmful effects in mirror reactors: They increase the electron density relative to the ion density; they radiate energy; and they scatter the ions.

The first two effects are shared with all fusion reactors; the third is significant because mirror machines have a loss cone. These effects are discussed below. In general, mirror reactors achieve high power densities and are in consequence relatively tolerant of the power losses due to impurities. The impurities in $2 X I I B$ enhanced the power deposited by the neutral beams; but this effect will be much smaller in reactors, because ionization processes becone more important than charge-exchange as the injection energy increases.

Impurities increase the electron density relative to the ion density, which can decrease the $i$ on confinement. If the density of totally stripped oxygen is $2 \%$ of the deuterium (and tritium) density, the electron density is 116\%. Titanium, which hes 25 electrons, has a much larger effect. The increased electron density increases the cooling of the ions. The ion confinement time should be limited by ion-ion scattering, and depends on the ion energy to the $3 / 2$ power. In consequence, cooling the ions decreases the ion confinement time. The detailed mechanisms by which this takes place would vary depending on the device.

Impurity radiation could limit the performance of mirror reactors, but these machines can tolerate reasonable impurity concentrations. Radiation power-10ss estimates are compared with other power densities of interest in Table 8. The radiation power density per percent impurity concentration is estimated as

$$
P=0.01 n_{e}^{2} L\left(W-\mathrm{cm}^{-3}\right)
$$




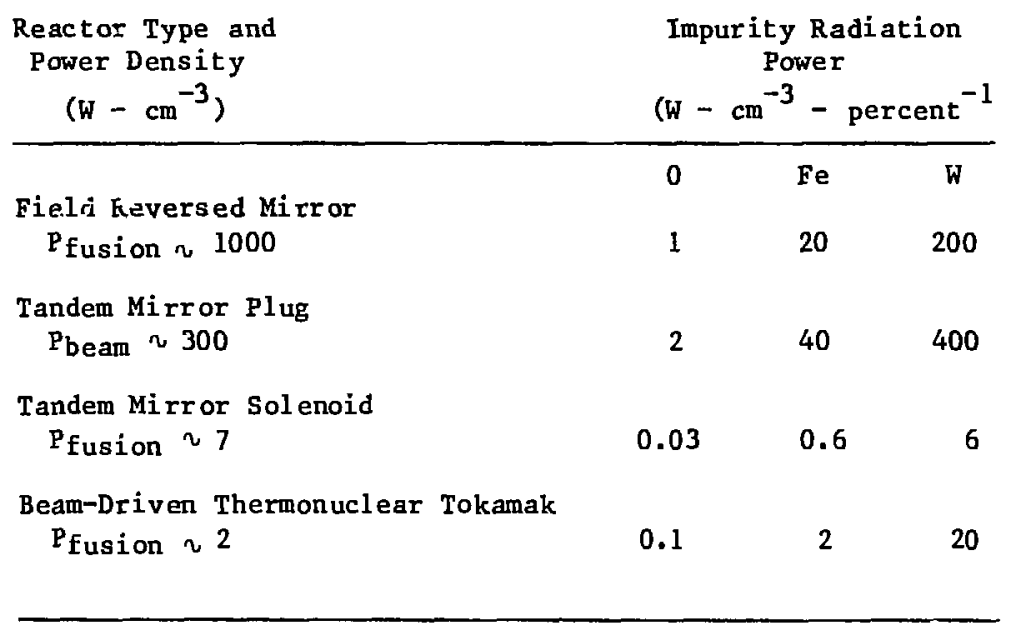

in which $L$ is taken from Post et a1. [40]. The fusion power densities were taken from recent reactor studies [41-43]. (For the tandem mirror reactor plug, the deposited neutral beam power is more relevant.) The field-reversed mirror reactor [41], which confines ions in a region of closed field lines, is quite tolerant of impurities because it has a tremendously high power density. The end plug of the tandem mirror reactor [42], which is used to prevent the loss of ions from a long solenoid, is so strongly heated by neutral beams that, as in $2 X I I B$, impurity radiation should not be a major problem. The solenoid of the tandem mirror reactor, which produces the fusion power in that device, is more sensitive to impurity radiation, but could apparently withstand radiation losses from a few percent of oxygen or a few tenths of a percent of iron (which is like titanium). It is seen ir. the next section that the tandem mirror experiment (TMX), which is intended to 
demonstrate the tandem mirror concept in a scalable geometry, may require lower impurity concentrations than the reactor will. For comparison, Table 8 al so shows power densities for a beam driven thermonuclear tokamak [ 43 ] (this device was ass umed to have a minor radius of $1 \mathrm{~m}$ ). The tokamak, because of its 1 ow fusion power density, would be more severely affected by a given concentration of imfurities than the mirror reactors would be.

The power lost from a mirror reactor plasma due to the scattering of deuterium ions into the loss cone by impurities should be mimportant, because of the energy dependences of Coulomb processes. The plasma potential should qui ckly eject 1 ow energy impurities, and high energy impurities have weak effects. The power loss per unit volume due to scattering is

$$
P_{10 s s}=n_{D}+E_{D}+\tau_{\text {scatt }} .
$$

Using the equation for the scattering lifetime from Table 2, (which may underestimate $\tau_{\text {scatt }}$ for high energies), and assuming a deuterium plasma, we find

$$
P_{10 s s}=\frac{N\left(n_{D}\right) J M z_{\text {imp }}^{2} \times 1.6 \times 10^{-19}}{5 \times 10^{10} J_{E_{\text {imp }}} 2 \log _{10} R_{m}},
$$

in which $N, M, Z_{i m p}$, and $E_{i m p}$ are the density, mass (amu), charge, and energy (keV) of the impurity. Assuming the effective mirror ratio, $R_{m}$, to be 2 , the power loss per unit volume per percent impurity concentration is

$$
P_{\text {scatt }}=0.01 \mathrm{n}_{\mathrm{D}^{2}}^{2} \times 5 \times 10^{-30} \frac{\mathrm{W} \mathrm{z}^{2}}{\mathrm{E}_{\mathrm{imp}}} \equiv 0.01 \mathrm{n}_{\mathrm{D}^{+}}^{2} \mathrm{Q} .
$$


As the impurity energy must be well above the electron temperature ( $50 \mathrm{keV})$ For the impurities to penetraze the plasma, we can estimate $E_{i m p} \sim 100 \mathrm{keV}$ and $M \sim 56$ (iron) to get

$$
Q \sim 4 \times 10^{-30} \mathrm{z}^{2}\left(W-\mathrm{cm}^{+3}\right) \text {. }
$$

The comparable factor for radiation power loss L, defined above, is

$$
L \simeq 7.4 \times 10^{-30} \mathrm{z}^{2}\left(\mathrm{~W}-\mathrm{cm}^{3}\right)
$$

for electron temperatures in this range (based on Ref. [40]). It is therefore seen that, in mirror reactors, losses due to scattering should not be 1 arge by comparison with those due to radiation.

C. EUV Diagnosis of the Tandem Mirror Experiment (TMX)

We begin our discussion of the possible EUV diagnosis of TMX by evaluating the potential impurity problems of this experiment. Then, the ways in which EUV diagnostics could help evaluate other aspects of tandem mirror operation are discussed.

The tandem mirror experiment is composed of two 2XIIB-type mirror machines (plugs) connected by a 5-m sol enoidal section [ 44$]$. The plugs should be like 2XIIB, and, like 2XIIB, should not suffer from impurityrelated difficulties. The deuterons in the solenoid will be well-confined by an electrostatic well. Impurities should also be we1l-confined in the solenoid, and this may lead to difficulties.

The fraction of impurities in the solenoid necessary to radiate all the power deposited in the TMX end plugs cais be estimated. Balancing power input to radiated output:

$$
r_{\text {iep }}=I_{\text {trap }} E_{\text {trap }}=P_{1 \text { oss }}=n_{c}^{2} v_{c} \text { Lf }
$$


As the impurity energy must be well above the electron temperature ( $250 \mathrm{keV}$ ) for the impurities to penetraze the plasma, we can estimate $E_{i m p} \sim 100 \mathrm{keV}$ and $M \sim 56$ (iron) to get

$$
Q \sim 4 \times 10^{-30} z^{2}\left(W-\mathrm{cm}^{+3}\right)
$$

The comparable factor for radiation power loss $L$, defined above, is

$$
L=7.4 \times 10^{-30} \mathrm{z}^{2}\left(W-\mathrm{cm}^{3}\right)
$$

for electron temperatures in this range (based on Ref. [40]). It is therefore seen that, in mirror reactors, losses due to scattering should not be large by comparison with those due to radiation.

\section{EUV Diagnosis of the Tandem Mirror Experiment (TMX)}

We begin our discussion of the possible EUV diagnosis of TMX by evaluating the potential impurity problems of this experiment. Then, the ways in which EUV diagnostics could help evaluate other aspects of tandem mirror operation are discussed.

The tandem mirror experiment is composed of two 2XIIB-type mirror machines (plugs) connected by a 5-m solenoidal section [ 44]. The plugs should be like $2 X I I B$, and, like $2 X I I B$, should not suffer from impurity related difficulties. The deuterons in the solenoid will be well-confined by an electrostatic well. Impurities should also be well-confined in the solenoid, and this may lead to difficulties.

The fraction of impurities in the solenoid necessary to radiate all the power deposited in the TMX end plugs can be estimated. Balancing power input to radiated out put:

$$
P_{\text {dep }}=I_{\text {trap }} E_{\text {trap }}=P_{1 \text { oss }}=n_{c}^{2} v_{c} \text { Lf }
$$


In this equation, the deposited power $(480 \mathrm{~kW})$ is the trapped beam current (16 A) times the average energy of trapped particles ( $230 \mathrm{keV}$ ) [45] and the power radiated, using Eq. (1), is the square of the sol enoid density $\left(n_{c}=1,2 \times 10^{13} \mathrm{~cm}^{-3}\right.$ ) times the impurity fraction (f), the solenoid volume $\left(v_{c}=1.7 \times 10^{6} \mathrm{~cm}^{3}\right)$, and the cooling rate $(L)$. The cooling rate depends on the el ectron temperature Light impurities (such as oxygen radiate strongly at 1 ow temperatures $\left(\mathrm{T}_{\mathrm{e}} \sim 50 \mathrm{eV}\right)$, but as $\mathrm{T}_{\mathrm{e}}$ approackes $200 \mathrm{eV}$, their radiated power becomes insignificant. By contrast, titanium radiates strongly at all temperatures expected in TMX. If the electron temperature is $50 \mathrm{eV}$, a $2 \%$ concentration of light impurities would radiate all the deposited power $\left(L \sim 10^{-25} \mathrm{~W}-\mathrm{Cl}^{3}\right)$. With an electron temperature of $200 \mathrm{eV}$, the light impurity radiation will be insignificant (except possibly from the plasma boundary), but a titanium concentration of $4 \%$ would radiate all the deposited power. These simple estimates make it clear that impurity radiation power loss could be a major factor in the TMX power balance.

The sources of impurities in TMx are worthy of consideration. Ions which have been magnetically trapped in the end plugs should seldom flow into the solenoid, as a result of detailed considerations of ian confinement [42]. This means that the neutral-beam-injected axygen should flow out of the machine, and should otherwise behave very much as it does in 2 XIIB (see Section V). Impurities generated at the end walls of TMX should be kept out of the solenoid by the high potential of the plugs, and should in consequence cause negligible power 1 oss. In contrast, impuritias produced by sputtering from the radial walls of MX may be deposited in the solenoid, if they are ionized between the midplanes of the two plugs. The carbon and titanium in 2XIIB may have resulted from wall-sputtering (see Appendix F). In consequence, it is possible that carbon and titanium sputtered from the radial walls of TMX will radiate substantial power. 
The impurity radiation from the solenoid can be measured using EUV spectroscopy, and several other aspects of tandem mirror physics can also be investigated. Table 9 summarizes useful EUV instrumentation for TMX. These instruments either exist, are under development, or are straightforward applications of proven designs. Some of the experiments they make possible will now be described:

(1) The time-resolving spectrograph (now under development at Johns Hopkins) will be able to measure many lines simultaneously. The time development of impurity concentrations and power loss can then be determined on each shot. The effects of various techniques of machine operation on impurity concentrations and power loss can be evalsated, and shots in which impurities are significant can be readily identified.

(2) The addition of a spectrometer on an end plug makes possible the evaluation of two additional effects. Whether particles trapped in the plug do flow only out the end of the machine, as predicted, can be evaluated by injecting an impurity in the neutral beams. The axial confinement of highly charged impurities can also be tested by introducing impurities in the solenoid.

(3) If the spectrometer on the plug has the capacity to measure at least three oxygen emission lines simultaneously, it can be used to monitor the plug oxygen and also to measure the time development of the electron temperature by the method described in Section $V$.

(4) A spatial-imaging and time-resolving monochromator will be installed on the THX solenoid. This instrument can provide information about the electron temperature-profile (assuming the expected electron-density data is available), by determining the shell structure of the impurities. In addition, it can evaluate the radial transport of impurities during impurity injection experiments. 
TABLE 9. TISEFUL EUV INSTRUMENTATION FOR TMX

\begin{tabular}{lll}
\hline \multicolumn{1}{c}{ Instrument } & Location & Applications \\
\hline Time Resolving Spectrograph & Solenoid & Concentrations and Power Loss \\
Spatial Imaging Monochromator & Solenoid & Impurity Transport, Te Profiles \\
High Resolution Spectrometer & Solenoid & Impurity Ion Temperatures \\
Three Channel oxygen Spectroneter & Plug & T Versus Time, Oxygen Studies \\
Lyman Alpha Spectrometer & Movable & Cold Gas Fluxes \\
Single Channel Monochromator & Movable & Transport studies, Precision Photometry
\end{tabular}


(5) A high-resolution spectrometer, using a vibrating mirror or plate, can measure the impurity ion temperatures in the end plug or solenoid. As the impurities should be in thermal equilibrim with the ions and electrons in the solenoid, this can be an ion temperature measurement. In the plug, the velocity distribution of the beam injected oxygen ions may indicate what processes are affecting them.

(6) A Lyman al pha spectrometer can directly measure the cold neutral flux to the plasma, if the flux of high energy neutrals is small at a given place or time. This can help evaluate the particle and energy bal ance of the plasma, as well as the problem of wall reflux.

(7) Finally, impurity injection experiments, diagnosed using the EUV instrumentation just described, can be performed to study transport mechanisms. By appropriate choice of impurity species and energy, and of TMX operating conditions, such experiments may allow us to test theories of transport across field lines, which are very important for alpha particle transport and impurity removal in reactors.

\section{EUV Diagnosis of the Mirror Fusion Test Facility (MFTF)}

The Mirror Fusion Test Facility (MFTF) is a single-cell mirror machine that is much 1 arger than 2 XIIB [46]. It is designed to test the scaling of ion confinement in mirror devices; EUV diagnostics can be used to study four different parts of this machine. These are the central plasma, the radial boundary, the region just inside the mirrors, and the end fans outside the mirrors. They are considered in turn.

The central plasma in MFTF is expected to be dense $\left(\mathrm{n}_{\mathrm{e}} \sim 10^{14} \mathrm{~cm}^{-3}\right.$ ) and hot $\left(\mathrm{T}_{i} \sim 50 \mathrm{keV}, \mathrm{T}_{\mathrm{e}} \sim 700 \mathrm{eV}\right)$. The mechanisms which all ow titani um and carbon to exist in 2 XIIB are not understood, and these elements should be 
studied to evaluate possible problems in a reactor. Given the expected electron temperature, a grazing incidence spectrograph is needed to effectively study impurities in the central plasma (a grazing-incidence time-resolving s pectrograph would be desirable, but may not exist soon enough). In addition, a high-resolution spectrometer could monitor the velocity distribution of the beam injected oxygen as plasma conditions (density, temperature, f1uctuation level) are changed. As a result, the ability to model the processes that affect energy diffusion could be tested.

The radial plasma boundary is important because particles and energy can be transported through it, and knowledge of it affects the accuracy of global measurements. In addition, radial ion transport may be important in MFTF (ion confinement in 2XIIB is not well understood). Indications of the electron density and temperature profiles in this region may be obtained using a spatial imaging and time resolving monochromator. The effects of wall conditions and sputering on the gas and impurity fluxes to the plasma boundary can be investigated using a normal incidence time resolving spectrograph, which can monitor emissions from low ionization states and Lyman alpha in the boundary region.

The hot plasma does not extend all the way to the magnetic mirrors, and the intervening region is important for several possible stabilization schemes. If we can deposit particles in this region and by some mechanism prevent their escape through the mirrors, the stabilizing stream can be provided without excessive heat $10 s 8$ to the end walls. When this is tried, Lyman alpha measurements would help investigate the hydrogen dynamics, and the impurity emissions will indicate whether impurities then accumulate in this region. A normal-incidence monochromator and a Lyman al pha spectrometer, or a time-resolving spectrograph, can be used for this. 
Finally, the end-fan plasma, outside the mirror, is important because it conducts power away fran the central plasma. The accumulation of impurities there may aff ect both the electron power bal ance and the penetration of impurities into the central plasma. An EUV monochromator could be used to investigate these issues. 


\section{Al. Introduction}

As described in Section II, the ion velocities in 2 XIIB are about $20 \%$ of the electron velocities. As a result, ion collision processes were carefully evaluated. The results are reported here. In sumary, ion impact excitation and ionization are not important $f$ or most of the ionization states observed.

There exist three basic types of cross section estimates for ion impact collision processes [47]. Quantum mechanical estimates, based on the Born Approximation or simple perturbation theory, are too simple and do not agree well with the experimental data. Exact classical calculations do somewhat better, but overestimate the effects of long range collisions. The classical calculations of Gryzinski [ 48], as assessed by Stabler [49], come closest to agreement with the experimental data, as a result of an ad hoc assumtion made by Gryzinski. Gryzinski's calculations, which wre used here, are expected to agree with the actual cross sections to within a factor of 2 for ion velocities within a factor of 5 of the orbital-electron velocity. The important ion velocities in 2 XIIB are in this range. Although the cross section is less well known near threshold, it is known to be small.

The classical description of these collisions (which are primarily a binary Coulomb process) can be summarized. The target atom imposes a reference frame on the collision, within which the electrons and bombarding ions can have a wide range of velocities. The energy exchange due to collisions is largest when the speed of the bombarding ion almost equals that of the orbiting electron (assuming an isotropic distribution of relative velocities). The electron has a velocity given approximately by $1 / 2 \mathrm{~m}$ 
$v_{e}^{2}=U$, the ionization potential. The "Coulonb velocity function" [48] determines the basic shape of the cross section:

$$
f_{v}=\left(\frac{v_{e}}{v_{i}}\right)^{2}\left(\frac{v_{i}^{2}}{v_{i}^{2}+v_{e}^{2}}\right)^{3 / 2}
$$

\section{A2. Calculation Method}

The rate coefficients for these processes were evaluated using the expression

$$
\langle\sigma v\rangle=N \int \sigma v F(v) d^{3} v .
$$

The cross section is averaged over the electron velocities and collision angles, so $v$ is the velocity of the bombarding ion and $f(v)$ is its velocity distribution. $N$ is the number of electrons in the outer shell of the target ion. In 2XIIB, the only significant ion motion is perpendicular to the magnetic field, and there is azimuthal symmetry, so Eq. (Al) becomes

$$
\langle\sigma v\rangle=2 \pi N \int \sigma v^{2} f(v) d v
$$

in which $v$ is now velocity perpendicular to $B$, and $E(v)$ is normalized in two dimensions .

A loss-cone-velocity distribution was used for the $2 X I I B$ ions. It corresponds to the measured distribution and has al so been used by Turner [7]. It is given by

$$
f(v)=\frac{v^{2}}{\pi v_{H}^{4}} e^{-v^{2} / v_{H}^{2}}
$$


in which $v_{H}=(<E>/ M)^{1 / 2}$. Velocities bel ow $2 \times 10^{7} \mathrm{~cm} \mathrm{~s} \mathrm{~s}^{-1}(500 \mathrm{eV})$ and above $2 \times 10^{8} \mathrm{~cm} \varepsilon^{-1}(40 \mathrm{keV})$ were ignored.

\section{A3. Ionization}

The deuteron-impact ionization rate coefficients were calculated using the formulas of Gryzinski:

$$
\sigma=\frac{\sigma_{0}}{u^{2}} G
$$

and

$$
\begin{aligned}
G=f_{v}\left[\frac{v_{i}^{2}}{v_{i}^{2}+v_{e}^{2}}+\right. & \left.\frac{2}{3}\left(1-\frac{u}{\Delta E_{\max }}\right) \ln \left(2.7+\frac{v_{i}}{v_{e j}}\right)\right] \\
& x\left(1-\frac{u}{\Delta E_{\max }}\right)\left[1-\left(\frac{u}{\Delta E_{\max }}\right)^{1+v_{i}^{2} / v_{e}^{2}}\right],
\end{aligned}
$$

in which

$$
\Delta E_{\max }=4 U\left(\frac{v_{i}}{v_{e}}\right)^{2}\left(1+\frac{v_{e}}{v_{i}}\right)
$$

is the maximum energy which can be transferred in such a collision.

$$
\left(\sigma_{0}=6.56 \times 10^{-14} \mathrm{ev}^{2} \mathrm{~cm}^{2} \text {.) For } \mathrm{U}>\Delta \mathrm{E}_{\max }, \sigma=0\right. \text {. This cross section was }
$$

substituted into Eq. (A2) and the integration was done numerically.

The rate coefficients are shown in Table Al. Electron impact ionization rate coefficients are al so given, for an electron temperature of $30 \mathrm{ev}$. As $\mathrm{T}_{\mathrm{e}}$ is usually higher, these are lower limits. In general, deuteron impact ionization is unimportant. However, ionization calculations for neur tral atoms and singly charged ions should consider deuteron effects. Only 
TABLE A1. ION IMPACT IONIZATION (OUTER SHELL)

\begin{tabular}{|c|c|c|c|c|}
\hline State & $\mathrm{U}_{\mathbf{i}}(\mathrm{eV})$ & $\mathbf{N}$ & $\begin{array}{c}\text { Ion Impact }{ }^{a} \\
<\sigma V>\end{array}$ & $\begin{array}{c}\text { Electron } \\
\text { Jmpact } \\
<\sigma \mathrm{v}\rangle \\
\mathrm{T}_{e}=30 \mathrm{eV}\end{array}$ \\
\hline D I & 13.6 & 1 & $4.91 \times 10^{-9}$ & $1.81 \times 10^{-8}$ \\
\hline $0 \mathrm{I}$ & 13.6 & 4 & $1.96 \times 10^{-8}$ & $3.98 \times 10^{-8}$ \\
\hline 0 II & 35.1 & 3 & $5.15 \times 10^{-10}$ & $6.49 \times 10^{-9}$ \\
\hline 0 III & 54.9 & 2 & $4.81 \times 10^{-11}$ & $1.33 \times 10^{-9}$ \\
\hline 0 IV & 77.1 & 1 & $4.13 \times 10^{-12}$ & $2.73 \times 10^{-10}$ \\
\hline $0 \mathrm{~V}$ & 113.9 & 2 & $6.72 \times 10^{-13}$ & $3.45 \times 10^{-11}$ \\
\hline $0 \mathrm{VI}$ & 138.1 & 1 & $6.97 \times 10^{-14}$ & $5.13 \times 10^{-12}$ \\
\hline Ne I & 21.6 & 6 & $6.40 \times 10^{-9}$ & $1.19 \times 10^{-8}$ \\
\hline $\mathrm{Ne}$ II & 41.1 & 5 & $4.45 \times 10^{-10}$ & $2.81 \times 10^{-9}$ \\
\hline Ne III & 63.5 & 4 & $4.69 \times 10^{-11}$ & $9.44 \times 10^{-10}$ \\
\hline Ne IV & 97.0 & 3 & $3.08 \times 10^{-12}$ & $1.74 \times 10^{-10}$ \\
\hline $\mathrm{Ne} \mathrm{V}$ & 126.3 & 2 & $3.00 \times 10^{-13}$ & $2.84 \times 10^{-11}$ \\
\hline Ne VI & 157.9 & 1 & $1.85 \times 10^{-14}$ & $3.96 \times 10^{-12}$ \\
\hline
\end{tabular}


these species, which have low ionization polentials, contain electrons that move slowly enough to be significantly affected by the deuterons in 2 XIIB. More highly ionized species, such as $0 \mathrm{VI}$, would only be affected by ions moving much faiter than those in 2 XIIB.

Table A2 shows estimates of double ionization rate coefficients using the approximation $\langle o v>\sim \sigma(v) \cdot \vec{v}$. Double ionization is the process in which two electrons are liberated as the result of the interaction of a target atom with one bombarding particle. For all ions in 2 XIIB this process is quite small; however, it may be significant for neutral atoms. The cross section is estimated assuming an atomic radius of $1 \stackrel{\circ}{A}$, and the dominant effect it includes $(80 \%)$ is second ionization by the bombarding ion [48], in which the ion frees two electrons in successive collisions.

TABLE A2. DOUBLE IONIZATION BY ION IMPACT

\begin{tabular}{lccc}
\hline State & $\mathrm{n}_{\mathrm{e}}$ & $\begin{array}{c}\mathrm{U}_{1}, \mathrm{U}_{2} \\
(\mathrm{eV})\end{array}$ & $\begin{array}{c}\sigma^{\overline{\mathrm{v}}} \\
\left(\mathrm{cm}^{3} \mathrm{~s}^{-1}\right)\end{array}$ \\
\hline O I & 8 & $13.6,35.1$ & $2.9 \times 10^{-8}$ \\
Ne I & 10 & $21.6,44$ & $9.5 \times 10^{-9}$ \\
O II & 7 & $35.1,54.9$ & $3.7 \times 10^{-10}$
\end{tabular}


The classical computation of excitation rate coefficients is more complicated. The cross section results from an integral,

$$
\sigma=\int_{u_{k}}^{u_{k+1}} \sigma_{E}(v) d E
$$

in which $\sigma_{E}(v)$ is the differential cross section for transfer of energy $E$. $\mathrm{U}_{k}$ and $\mathrm{U}_{\mathrm{k}+1}$ are the excitation energies of the transition under consideration and the next transition of higher energy. The differential cross section is

$$
\sigma E=\frac{\sigma_{0}}{E^{3}} G^{\prime}
$$

in which

$$
G^{\prime}=f_{v}\left[\frac{v_{i}^{2}}{v_{i}^{2}+v_{e}^{2}} \frac{E}{u}+\frac{4}{3} \ln \left(2.7+\frac{v_{i}}{v_{e}}\right)\right]\left[1-\left(\frac{E}{\Delta E_{\max }}\right)^{1+v_{i}^{2} / v_{e}^{2}}\right]
$$

The numerical integration of Eq. (A3), computing $\sigma$ for each velocity using Eq. (A4), gave values $f$ or $<\sigma v>$. Table A3 shows these results, and compares then with typical electron impact excitation rate coefficients. It is seen that although deuteron impact excitation may contribute, its effect is small compared to the uncertainties in the electron impact excitation rat: coefficients. These rate coefficients also are used in calculations that introduce significant additional uncertainties. As a consequence, deuteron impact excitation was not included in the analys is of these data. 
TABLE A3. DEUTERON IMPACT EXCITATION RESULIS

\begin{tabular}{|c|c|c|c|c|}
\hline State & A & Feature & $\begin{array}{c}\left\langle\sigma v^{\mathrm{ex}}\right. \\
\mathrm{D}^{+} \\
10^{-9} \mathrm{~cm}^{3} \mathrm{~s}^{-1}\end{array}$ & $\begin{array}{c}\langle a v\rangle^{\text {ex }} \\
{ }_{10^{-9}} \mathrm{~cm}^{3} \mathrm{~s}^{-1}\end{array}$ \\
\hline $0 \mathrm{VI}$ & 1034 & Doublet & 4.21 & 28.0 \\
\hline $0 \mathrm{v}$ & 630 & Line & 2.70 & 29.5 \\
\hline $0 \mathrm{VI}$ & 554 & Mul tiplet & 2.08 & 13.1 \\
\hline $0 \mathrm{III}$ & 703 & Mul tiplet & 2.46 & 10.9 \\
\hline $0 \mathrm{II}$ & 539 & Multiplet & 0.43 & 4.1 \\
\hline $\mathrm{N} \mathrm{V}$ & 1240 & Doublet & 7.31 & 36.3 \\
\hline C IV & 1548 & Doublet & 14.3 & 62.7 \\
\hline
\end{tabular}




\section{B1. Introduction}

The neutral-beam fueling of 2 XIIB establishes a large neutral density. This neutral density is significant because it results in a high chargeexchange rate. The charge-exchange rate affects population distributions and enters into the model of beam injection of oxygen (Section $V$ ).

As the calculations above involve averages over the central plasma, the average neutral density is needed. This density can be estimated using well known global currents and transit times. The total number of neutrals in the plasma is the product of the neutral current and the average transit time of these neutrals (or equivalently the average distance they travel divided by their mean velocity). Dividing by the plasma volume yields the average neutral density. This calculation is carried through below.

\section{B2. Calculation of Neutral Densities}

Three groups of neutral particles contribute to the neutral density. These are: (i) Neutral-beam atoms which pass through the plasma. (ii) Beam atoms which undergo charge-exchange. (iii) Beam atoms which are ionized.

(i) The transmitted component of the beam is

$$
I_{t}=I_{b} T=I_{b} e^{-\int n_{e} d l \sigma_{e f f} / 1.41}
$$

This formula is determined from the results of a computer code written by W. C. Turner which evaluates neutral-beam transmission.

$$
\left(\sigma_{\text {eff }}=1.54 \times 10^{-15} \mathrm{~cm}^{2} .\right)
$$


The central plasma is the region with $r \leqslant R_{p}$ (the reasons for this are explained in Appendix $c$ ). The average transit time is the length of the average chord traversed divided by the mean velocity. Thus

$$
t=\frac{R}{v}=\frac{2 R_{p} \sqrt{1-\left(y_{0} / R_{p}\right)^{2}}}{v}
$$

in which $y_{0}=$ the distance from the axis the beams are aimed. The quantity $\sqrt{1-\left(y_{0} / R_{p}\right)^{2}}$ vaxies slowly and has a typical value of $0.94\left(y_{0}=3 \mathrm{~cm}\right.$; $\left.R_{p}=8.5 \mathrm{~cm}\right)$. The plasma volume, $V$, is $\pi R_{p}^{2} L$ so

$$
\bar{n}_{t}=\frac{I_{b} T t}{v}=\frac{2 I_{b} T}{\pi v R_{p} L} \sqrt{1-\left(y_{0} / R_{p}\right)^{2}}=\frac{1.88 I_{b} T}{\pi v R_{p} L}
$$

(ii) The neutrals that undergo charge-exchange behave as follows: they penetrate some distance into the plasma, then charge-exchange, then either escape, ionize, or charge-exchange again. The latter two processes are complicated, but fortunately for the calculations they affect only a small fraction of the neutrals.

As may be seen in Fig. B1, the beam neutrals which are attenuated travel an average distance of about $0.7 \mathrm{R}_{\mathrm{P}}$ into the plasma first. After a charge-exchange event, the resulting neutral travels an average distance $\varkappa_{\mathrm{p}}$ before escaping. (This distance is reduced by ionization events but is increased by further charge-exchange.) The total average distance chargeexchange participants travel as neutrals in the plasma may be estimated as $(1.5 \pm 0.3) R_{p}$ 


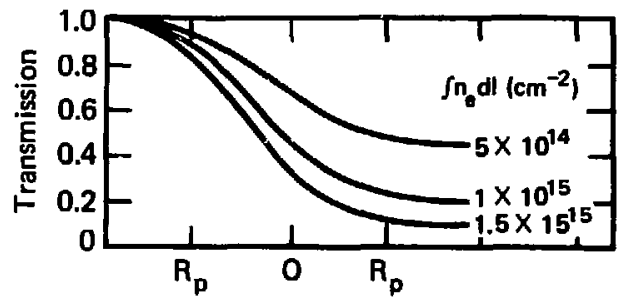

Position

Figure Bl. The transmission of the neutral beams through plasmas of various line densities is shown. A Gaussian spatial density profile was assumed.

Now one can write

$$
\begin{aligned}
& I_{c x}=f_{c x} I_{b}(1-T) ; f_{c x}=0.75 \\
& t_{c x}=\frac{(1.5 \pm 3) R_{P}}{v} ; V=\pi R_{p}^{2} L \\
& \bar{n}_{c x}=\frac{I_{b}(1-T) f_{c x} t_{c x}}{V}=\frac{f_{c x}(1.5 \pm 0.3) I_{b}(1-T)}{\pi v R_{p}}
\end{aligned}
$$

(iii) The neutral beam atoms which become ionized travel only the average initial distance $=(0.7 \pm 0.15) \mathrm{R}_{\mathrm{p}}$ into the plasma. Thus

$$
\begin{aligned}
& I_{\text {ion }}=f_{i} I_{b}(1-T)=\left(1-f_{c x}\right) I_{b}(1-T) \\
& t_{\text {ion }}=\frac{(0.7 \pm 0.15) R_{p}}{v} \\
& n_{\text {ion }}=\left(1-f_{c x}\right)(0.7 \pm 0.15) I_{b}(1-T) /\left(\pi v R_{p} L\right)
\end{aligned}
$$

The total average neutral density is the sum of these three components. This is 


$$
\begin{aligned}
& \bar{n}_{D}=\frac{I_{b}}{\pi v R_{p} L}\left[1.88 T+\left\{f_{c x}(1.5 \pm 0.3)\right.\right. \\
& \left.\left.\quad+\left(1-f_{c x}\right)(0.7 \pm 0.15)\right\}(1-T)\right] \\
& \bar{n}_{D}=(1.3+0.6 T) \frac{I_{b}}{\pi v R_{p} L} \pm 0.4(1-T) \frac{I_{b}}{\pi v R_{p} L}
\end{aligned}
$$

The resulting values of $\bar{n}_{D}$ for three days of interest are shown in Table B1. L was taken to be $36 \mathrm{~cm}$.

TABLE BI. NEUTRAL DENSITIES IN 2XIIB

\begin{tabular}{llll}
\hline Date & $n_{D}\left(\mathrm{~cm}^{-3}\right)$ & $\pm\left(\mathrm{cm}^{-3}\right)$ & $\mathrm{T}$ \\
\hline $6 / 14 / 78$ & $4.3 \times 10^{10}$ & $0.7 \times 10^{10}$ & 0.40 \\
$10 / 24 / 77$ & 4.0 & 0.8 & 0.24 \\
$3 / 8-9 / 78$ & 3.8 & 0.4 & 0.57 \\
\hline
\end{tabular}

B3. Scaling of the Neutral Density

Equation (B1) can be evaluated to determine the neutral density scaling. The term $(1.3+0.6 \mathrm{~T})$, which depends only on line density, is fit very well as

$$
1.3+0.6 \mathrm{~T}=2.07{\frac{\int \mathrm{n}_{\mathrm{e}} \mathrm{dl}}{10^{14}}}^{-0.14},
$$


for $5 \leq \frac{\int n_{e} d l}{10^{14}} \leq 15$. This means that one has (with $L=36 \mathrm{~cm}, v=10^{8} \mathrm{~cm} / \mathrm{s}$ )

$$
\bar{n}_{D}=1.4 \times 10^{9}\left(\frac{\int n_{e} d 1}{10^{14}}\right)^{-0.14} \frac{I_{b}}{R_{p}}
$$

witn $I_{b}$ in A. Equation (B2) is a general scaling which can be applied to almost all 2 XIIB data.

This general scaling can be applied to the $6 / 6 / 78$ data, for which a model of $O$ II emissions is constructed in Appendix $E$. We have $R_{p} \simeq 10 \mathrm{~cm}$ and

$$
\int n_{e} d l=1.5 \times 10^{12} I_{b}^{1.1}
$$

This yields

$$
\overline{\bar{n}}_{\mathrm{D}}=2.1 \times 10^{8} \mathrm{I}_{\mathrm{b}}^{0.85}
$$

This expression is used in Appendix E. 


\section{Cl. Introduction}

This Appendix presents' the-basic impurity data, and some of the conclusions about concentrations and power loss that follow from them. Appendix $G$ discusses the neon injection experiments in a similar fashion. The results presented in this Appendix are summarized in Section IV. Section V discusses further experiments and analysis, which led to more subtle conclusions about the oxygen in $2 X I I B$.

\section{C2. Measurements of 2XIIB Impurity Emissions}

This section describes the basic measurements of impurities which were analyzed to determine impurity concentrations and power loss. The identified lines, EUV emission characteristics, and unresolved emission lines are described. Then the absolute brightness measurements are reported. Finally, the spectral scans and spatial scans are sumarized.

\section{Observed Transitions}

The emission lines of oxygen, nitrogen, carbon, titanium and deuterium observed on 2 XIIB are tabulated in Table $\mathrm{Cl}$. The observed transitions are indicated; they are all either ground state resonance trausitions or resonance transitions to low lying metastable states. Except for the titanium, these transitions had all been observed previously with this equipment. Brief spectral scans verified the continuity of the wavelength calibration. Figure Cl shows some of the spectral data which allowed us to identify the Ti transitions. This data shows the triangular line profiles expected using $2 \AA$ 
TABLE Cl. OBSERVED IONS AND TRANSITIONS

\begin{tabular}{|c|c|c|c|}
\hline Ion & $(\AA)$ & Trans & \\
\hline D I & $1216\left(\mathrm{~L}_{a}\right)$ & $1 s-2 p$ & ${ }^{2} s-{ }^{2} P$ \\
\hline $0 \mathrm{VI}$ & 1038 & $2 s-2 p$ & ${ }^{2} s-{ }^{2} P$ \\
\hline $0 \mathrm{VI}$ & 1032 & $2 s-2 p$ & ${ }^{2} \mathrm{~s}-{ }^{2} \mathrm{P}$ \\
\hline $0 \mathrm{~V}$ & 760 (m) & $2 s 2 p-2 p^{2}$ & ${ }^{3} p-{ }^{3} p$ \\
\hline $0 \mathrm{~V}$ & 630 & $2 s^{2}-2 s 2 p$ & ${ }^{1} s-{ }^{1} P$ \\
\hline $0 \mathrm{IV}$ & 789 & $2 s^{2} 2 p-2 s 2 p^{2}$ & ${ }^{2} P-{ }^{2} D$ \\
\hline $0 \mathrm{IV}$ & 554 & $2 s^{2} 2 p-2 s 2 p^{2}$ & ${ }^{2} P-{ }^{2} P$ \\
\hline 0 III & 703 & $2 s^{2} 2 p^{2}-2 s 2 p^{3}$ & \\
\hline 0 II & $718(\mathrm{~m})$ & $2 s^{2} 2 p^{3}-2 s 2 p^{4}$ & \\
\hline 0 II & 539 & $2 p^{3}-2 p^{2} 3 s$ & ${ }^{4} s-$ \\
\hline c IV & 1548 & $2 s-2 p$ & ${ }^{2} s-{ }^{2} P$ \\
\hline C III & 977 & $2 s^{2}-2 s 2 p$ & ${ }^{1} \mathbf{s}-$ \\
\hline $\mathrm{N} \mathrm{V}$ & 1239 & $2 s-2 p$ & ${ }^{2} s-$ \\
\hline N IV & $923(\mathrm{~m})$ & $2 s 2 p-2 p^{2}$ & $3_{p}-$ \\
\hline N III & 685 & $2 s^{2} 2 p-2 s 2 p^{2}$ & \\
\hline Ti VII & 522 & $3 s^{2} 3 p^{4}-3 s 3 p^{5}$ & \\
\hline Ti VI & 524 & $3 s^{2} 3 p^{5}-3 s 3_{p}^{6}$ & ${ }^{2} \mathrm{P}$ \\
\hline Ti IV & 779 & $3 d-4 p$ & \\
\hline
\end{tabular}

Unmarked transitions are ground state resonance transitions.

(m) - Metastable resonance transitions. 


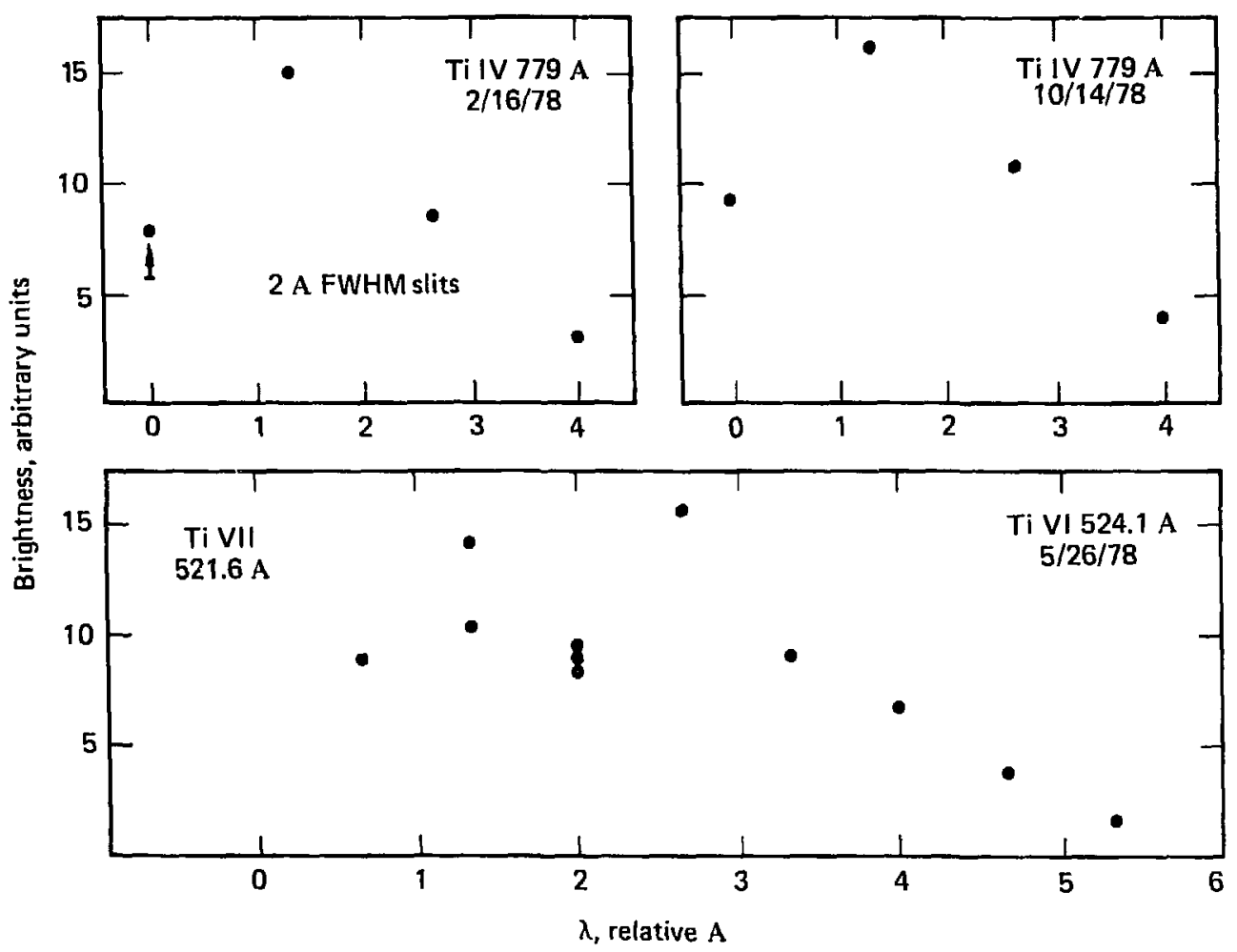

Figure Cl. Spectral scans of titanium emissions show the triangular $2 \&$ FWHM profile of the instrument transmission.

FWHM slits. These lines were scanned a number of times; their isolation was al so investigated by use of standard line tables [31].

The emission lines which were unresolved from the background signal are tabulated in Table C2, with upper limits on their brightnesses. A brightness of $10^{15} \mathrm{ph} \mathrm{s}^{-1} \mathrm{~cm}^{-2} \mathrm{sr}^{-1}$ corresponds to a concentration of less than $10^{-3}$. (This upper limit results from an assumed excitation rate coefficient of $10^{-9} \mathrm{~cm}^{3} \mathrm{~s}^{-1}$ and a line density of $6 \times 10^{14} \mathrm{~cm}^{-2}$, both of which are too low.) Copper emissions were not resolvable with this 
TABLE C2. EMISSION LINES UNRESOLVED FROM BACKGROUND

\begin{tabular}{lcc}
\hline State & Line (A) & $\begin{array}{c}\text { Maximum Plateau Brightness } \\
\left(\mathrm{ph} \mathrm{s}^{-1} \mathrm{~cm}^{-2} \mathrm{sr}^{-1}\right)\end{array}$ \\
\hline O I & 1304 & $2.4 \times 10^{15}$ \\
C II & 904 & $5 \times 10^{14}$ \\
N II & 916 & $1.7 \times 10^{15}$ \\
K VI & 716 & $1 \times 10^{15}$ \\
S IV & 1063 & $4 \times 10^{15}$ \\
C IV & 883 & $1.5 \times 10^{15}$ \\
W VI & 638 & $1 \times 10^{15}$ \\
\hline
\end{tabular}

equipment because of their spectroscopic structure. It is not know whether the neutral beams, which have copper arc chambers, inject copper into 2 XIIB.

\section{Brightness Measurements}

The time development of EUV emissions is described in Section IV. Most of the discussion below pertains to the steady-state, high-density phase of the plasma shot. The absolute brightness is the quantity directly measured by the EUV instrument. Some of the brightness data is tabulated in Table C3. The plasma parameters measured during the same shots are also shown. The most striking fact is that the EUV radiation from 2XIIB was very bright. The $0 \mathrm{~V}$ and $\mathrm{O} V \mathrm{~V}$ brightnesses reported in Table $\mathrm{C} 3$ were more than two orders of magnitude larger than those reported from Alcator [32], and an order of magnitude greater than those from TFR [ 33], yet these two tokamaks 
TABLE C3. SOME MEASURED BRIGHTNESSES AND PLASMA PARAMETERS

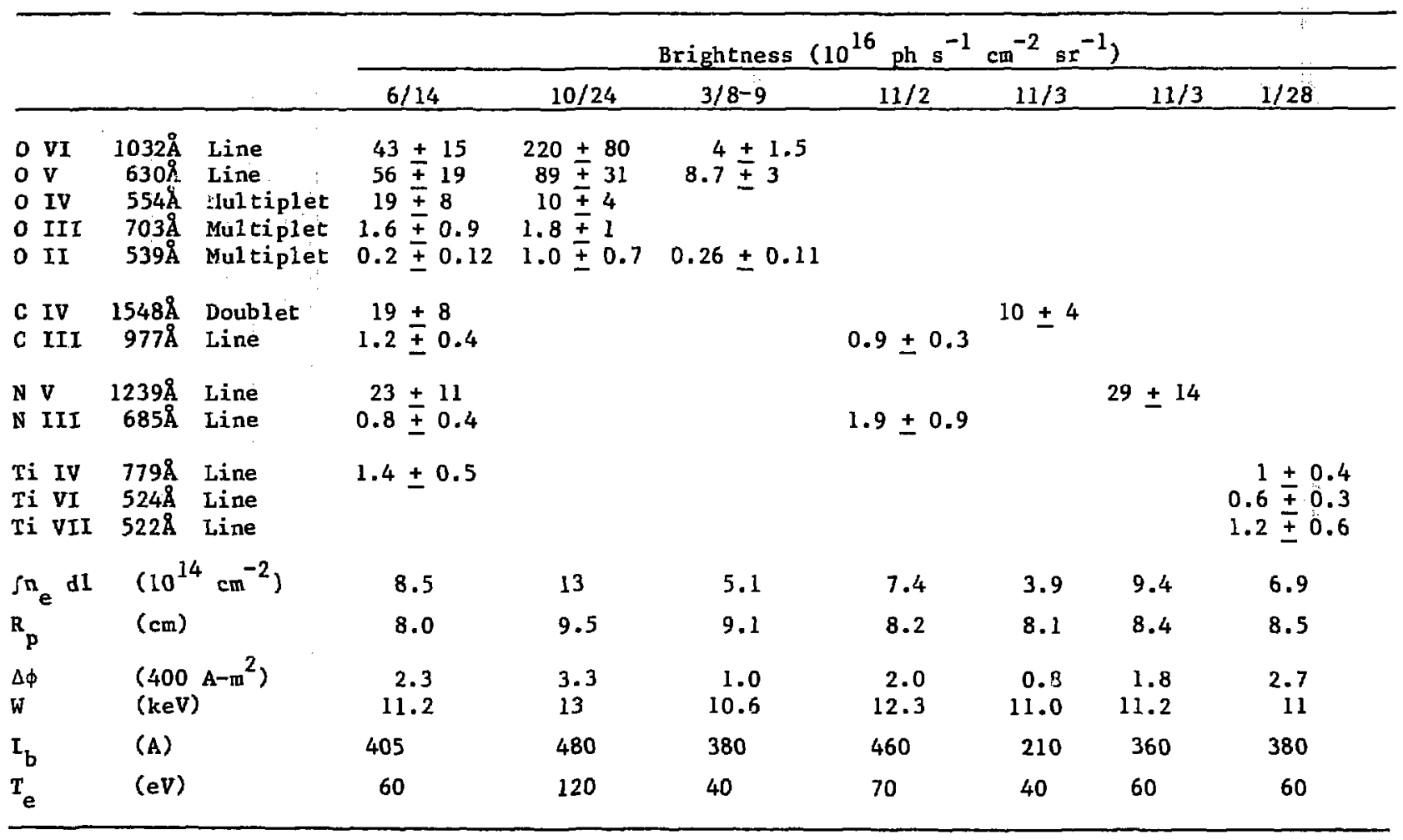


have a deuterium-confinement time more than ten times larger than that of 2XIIB. This indicates that the $2 X I I B$ impurity fluxes and/or the confinement times were relatively high.

The uncertainties in the reported brightnesses had several sources. The uncertainty in the calibration was included. Uncertainties in other terms of Eq. (7)(Section II), also were considered. Beyond that, there were uncertainties introduced by background subtractions and corrections for linebroadening. After these uncertainties were evaluated, the accuracy of a brightness measurement on one particular shot was known. Using this instrument, many shots must be compared to estimate concentrations or power loss, so the shot-to-shot $i_{i}$ reproducibility of 2 XIIB was taken into account. On the basis of experience, this effect is estimated as $\mathbf{+} 30 \%$--which includes changes in plasma parametors and impurity emissions. Within restricted sets of identical shots, this uncertainty is about $\pm 15 \%$, but the calculations presented here were intended to have more general applicability. Finally, in estimating a total uncertainty, the various sources of error were considered to be random.

\section{Machine Condtitions for These Measurements}

Impurity studies can be questioned if the data analyzed are not taken during representative machine operation. This objection does not apply to this study. The data presented in this work was obtained during the normal course of 2 XIIB experimentation. Some of the runs reported in Table c3 are discussed in reference [50]; the rest were also typical. For all the measurements reported above, the machine was pumped and gettered in the normal fashion, and stabilizing stream was supplied by standard methods. Ir most cases, data was being taken simultaneously for other purposes. 
In addition, EUV emission brightnesses from similar plasmas were found to vary little (except for emissions from states strongly affected by $T_{e}$ variations). 0 V $630 \AA$ emissions were frequently monitored; and measurements of this line on many days are tabulated in Table 44 . It is observed that, for similar electron line densities, $0 \mathrm{~V}$ brightness variations were generally within a factor of 2 . The orly exception to this was March 8, 1978, when o V emissions were relatively small, presumably due to the very low electron teriserature (see Section V).

\section{Spectral and Spatial Scans}

Spectral scans aided line identification and brightness measurements; some were complete enough to determine impurity energies from line broadening. Spectral scans were performed by stepping the wavelength drive through a line on a shot-by-shct basis. One angstrom slits were ordinarily used; and these scans were limited by the number of shots available with constant machine parameters. Doppler broadening is the only significant broadening mechanism in these plasmas; this allowed impurity energies to be determined from the measured line widths.

Figure C2 shows a spectral scan of the C III 977 A emissions. The target plasma emissions were $40 \%$ as bright as the emissions during the highdensity plateau, at the peak of the spectral line. These data are significant because the line becomes broad during the shot; it is quite narrow in the target plasma, before the neutral beams fire. (This was also observed in a second scan taken another day.) It shows that the observed broadening is real and is not due to systematic errors. Figures $\mathrm{C} 3$ and $\mathrm{C} 4$ show spectral scans of oxygen lines. 
TABLE C4. BRIGHTNESS OF O V $630 \AA$ ON VARIOUS DAYS

$$
\begin{aligned}
& D=\text { Date } \quad S=\text { Shot } \\
& B=\text { Brightness }\left(10^{17} \mathrm{ph} \mathrm{s}^{-1} \mathrm{~cm}^{-2} \mathrm{sr}^{-1}\right) \\
& \mathrm{N}=\text { Electron Line Density }\left(10^{14} \mathrm{~cm}^{-2}\right)
\end{aligned}
$$

\begin{tabular}{cccccccc}
\hline $\mathrm{D}$ & $\mathrm{S}$ & $\mathrm{B}$ & $\mathrm{N}$ & $\mathrm{D}$ & $\mathrm{S}$ & $\mathrm{B}$ & $\mathrm{N}$ \\
\hline $9 / 23$ & 20 & 12 & 7 & $5 / 19$ & 8 & 4.3 & 4.8 \\
$10 / 20$ & 38 & 9.1 & 13 & $5 / 21$ & 18 & 7.7 & 7.5 \\
$10 / 24$ & 25 & 10 & 15 & $6 / 1$ & 9 & 7.3 & 8 \\
$10 / 28$ & 32 & 13 & 18 & $6 / 6$ & 16 & 12 & 10 \\
$11 / 3$ & 36 & 6.9 & 7 & $6 / 9$ & 19 & 10 & 10 \\
$12 / 21$ & 26 & 19 & 15 & $6 / 13$ & 18 & 15 & 12 \\
$12 / 22$ & 7 & 22 & 15 & $6 / 14$ & 16 & 5.7 & 10 \\
$1 / 6$ & 19 & 3.1 & 4 & $6 / 20$ & 26 & 2.7 & 8 \\
$1 / 11$ & 7 & 2.5 & 3 & $6 / 22$ & 10 & 6.0 & 9 \\
$2 / 9$ & 18 & 3.0 & 4 & $6 / 27$ & 22 & 8.6 & 10 \\
$2 / 10$ & 31 & 2.8 & 4 & $7 / 14$ & 11 & 6.3 & 10 \\
$2 / 15$ & 16 & 5.2 & 6 & $7 / 15$ & 12 & 9.3 & 12 \\
$3 / 8$ & 5 & 0.87 & 5 & $7 / 16$ & 7 & 6.4 & 6 \\
$4 / 7$ & 7 & 4.7 & 8 & $7 / 27$ & 21 & 9.3 & 9 \\
$4 / 11$ & 8 & 1.4 & 4 & $7 / 29$ & 21 & 9.0 & 6 \\
$4 / 27$ & 13 & 2.3 & 5 & $7 / 31$ & 23 & 5.3 & 7 \\
& & & & & & & \\
\hline & & & & & & &
\end{tabular}




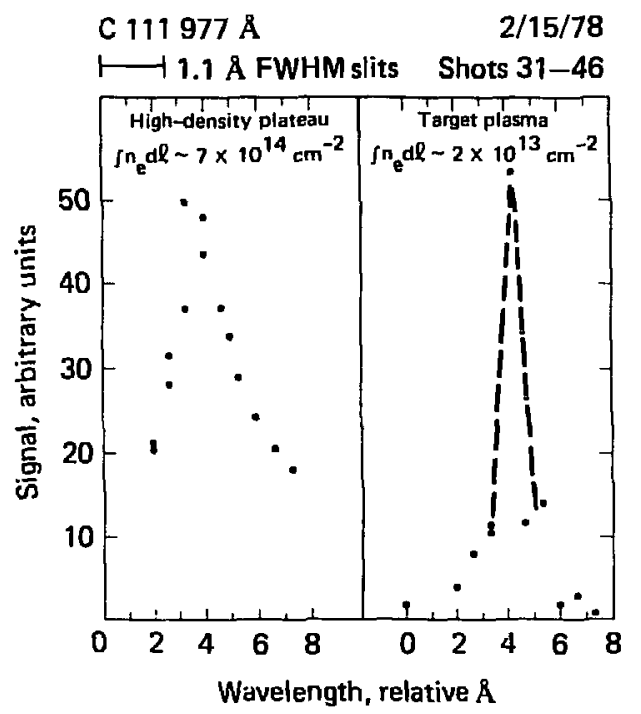

Figure C2. A spectral scan of C III $977 \AA$ emissior.s, showing that the line becomes broad during the shot. 


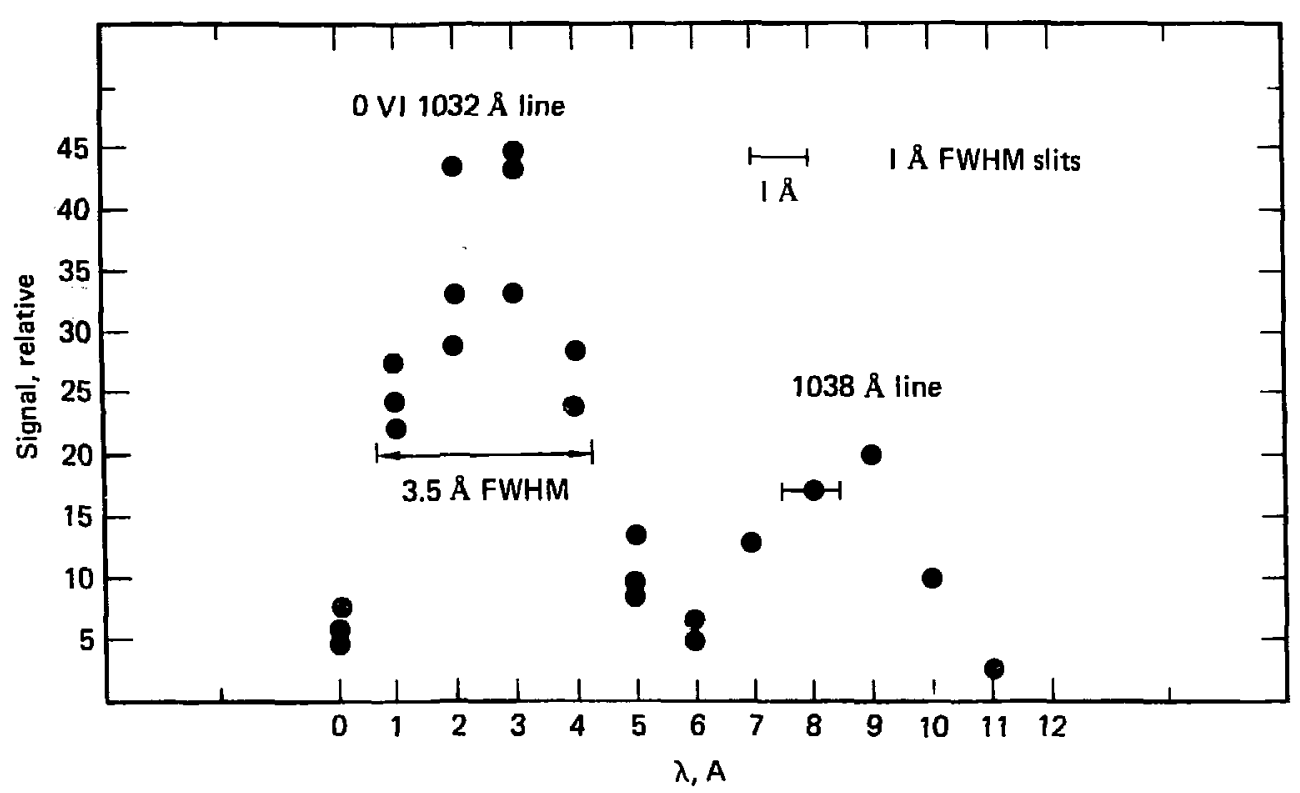

Figure C3. A spectra1 scan of the 0 VI $1034 \AA$ doublet. Doppler broadening and the expected 2:1 brightness ratio were niserved.

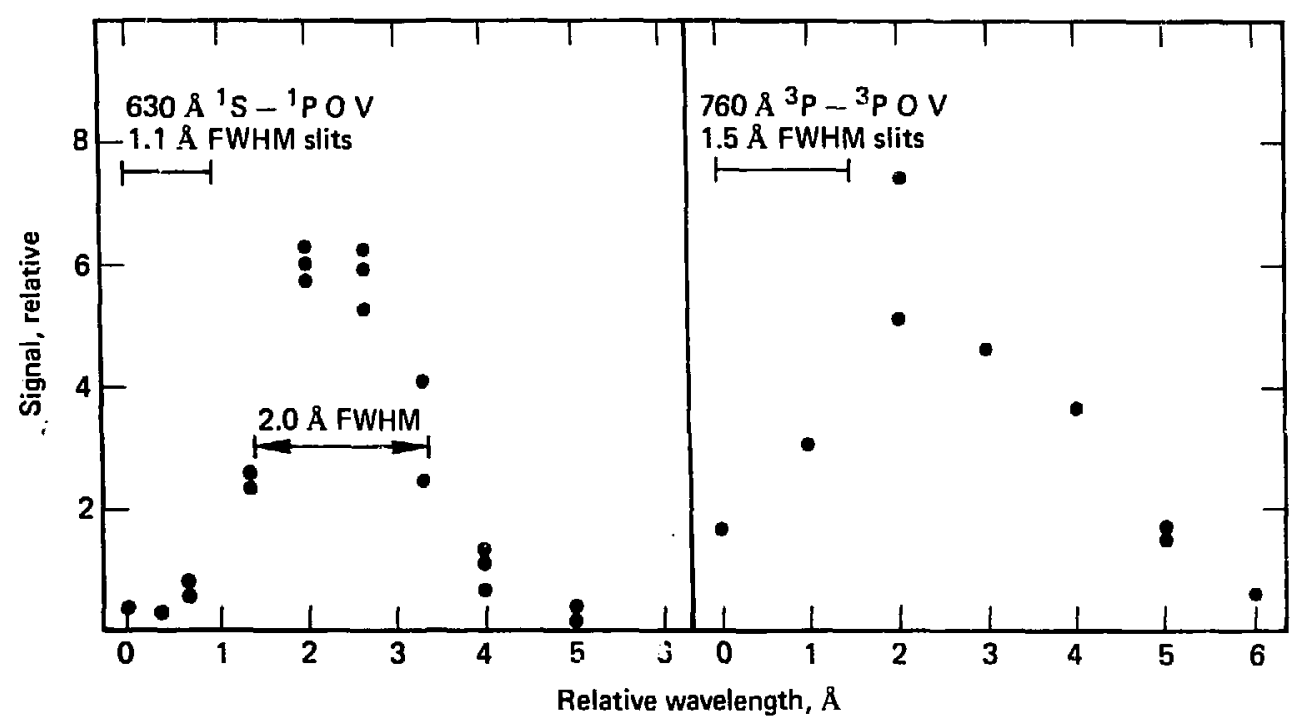

Figure C4. Spectral scans of $0 \mathrm{~V}$ emissions also showed substantial Doppler broadening. 
The energies cos responding to the observed broadening are given in Table 3. These energies correspond to the velocity necessary to emit a photon at the wavelength that is the half-maximum above background of the observed spectral profile. To unfold the instrumental broadening from the observed linewidth, the actual linewidth was computed as the root of the difference of the squares of these quantities. The profiles are assumed to be Gaussian. The uncertainties introduced by this approximation are less than those in the measured FWHM. The spectral scans of three different oxygen lines, made on separate days, are consistent. The energy at FWHM is about $15 \mathrm{keV}$, the mean oxygen energy (assuming a Gaussian profile) is about $10 \mathrm{keV}$. The significance of the observed carbon energy is considered in Appendix F; these data are not well understood. The N V $1242 \AA$ line was scanned once, and was found to be quite broad $(\sim 4 \AA)$. However, machine conditions were somewhat variable and this measurement was never repeated; accordingly this result must be considered tentative.

Spatial scans were performed by tilting the instrument about a point in the beamline on a shot-by-shot basis. These scans ...re used to distinguish among the constant concentration, constant density, and shell models discussed in Section III-E.

The impurity-density profile can in principle be determined by Abelinverting the observed brightness profile to obtain the volume emission profile; then dividing by the electron density and the excitation rate coefficient see Eq. (4) in Section III . The data obtained from $2 X I I B$ does not allow a precise determination of the impurity density profiles, because of irreproducibility. However, the inversions of various curves drawn through the data may be used to place limits on the spatial structure. 
Some 0 V $630 \AA$ spatial data were presented and discussed in Section IV (Fig. 7). This scan (and other O V spatial data) indicate that the constant concentration model (Section III-E) is appropriate for highly ionized oxygen. This model was used to analyze the $0 \mathrm{IV}, 0 \mathrm{~V}$, and $0 \mathrm{VI}$ data. All other states were analyzed using the constant density mode1, which was the best fit to spatial scans of 0 II $539 \AA$, C III $977 \AA$, N III $685 \AA$, and Ti IV $779 \AA$. The upper section of Fig. $C 5$ shows the peak brightness, the brightness profile and the line density profile during a C III spatial scan. The lower

\section{2/16/78 Shots 3-15}

Peak C III 977 \& B $=4.6 \times 10^{15} \mathrm{ph} \mathrm{s}^{-1} \mathrm{~cm}^{-2}$

$\mathrm{sr}^{-1} \int \mathrm{n}_{\mathrm{e}} \mathrm{dl}=6.7 \times 10^{14} \exp \left[-\mathrm{y}^{2} / 10^{2}\right] \mathrm{cm}^{-2}$

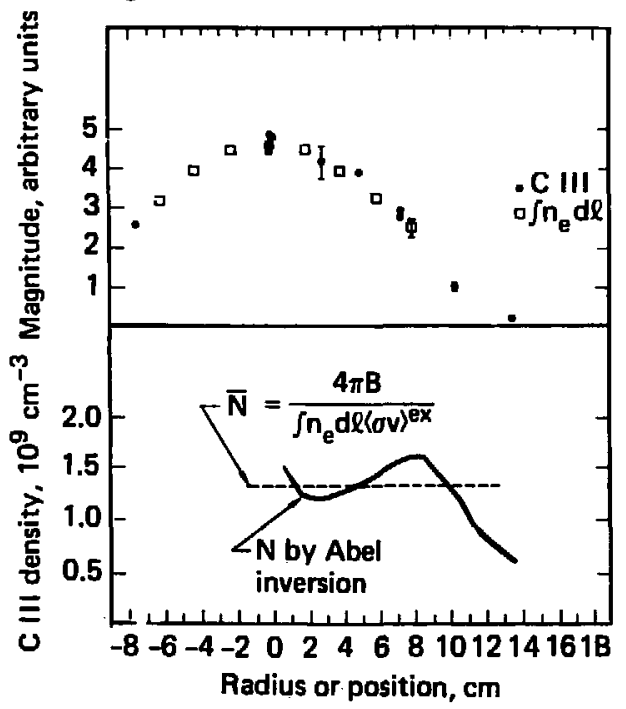

Figure C5. Spatial profiles of C III $977 \AA$ brightness and electron line density are shown above. A density profile obtained by Abel inversion and the density given by the constant density model (dashed line) are shown below. 
portion shows the constant-density model (dashed line) and the inverted profile obtained from hand-drawn "best fit" curve through the data. The best $O$ II scan, which is important in the argument of Section $v$, is discussed there. Additional spatial data may be found in Appendix D. Numerous scans show that emissions from the native impurities in 2XIIB have horizontal s unmetry.

The models selected above apply only to impurities in the central plasma ( $r \leqslant R_{p}$ ), and near the midplane along the machine axis. Because the electron density and temperature are not well known, the impurity spatial proriles at large radii $\left(r>R_{p}\right)$ are unknown. Some implications of this fact for further analysis are discussed in the next section.

\section{C3. Impurity Concentrations in 2XIIB}

Having proceeded from line identification to modeling of the plasma, impurity concentrations could be calculated reasonably. This section discusses these calculations and presents their results (summarized in Table 4). Some observations of plasmas created by one type of streaming plasma gun are aíso reported.

Concentrations in Beam Fueled Plasmas

The calculation of impurity concentrations is summarized for the constant density model. If the constant concentration model applies, the coefficients are slightly different but the basic dependences remain the same. The density of an impurity throughout the region $r \leqslant R_{p}$ is given, in this model, by

$$
N=\frac{4 \pi B}{\langle\sigma v\rangle^{e x} \int n_{e} d l}
$$


To evaluate equation (Cl), a value of $\mathrm{T}_{\mathrm{e}}$ must be used. This is not significant for this application, as these rate coefficients seldom vary more than $20 \%$ over the entire range of electron temperatures in 2XIIB.. In contrast, the average electron density must be known to compute the impurity concentration. The problem of determining $\bar{T}_{e}$ and $\overline{\mathbf{n}}_{e}$ will be considered here.

The central plasma in 2 XIIB is well diagnosed. The density and temperatures are we11 known near the midplane and at radii $r \approx \mathbf{R}_{\mathbf{p}}$. The impurity ata is not precise enough to specify impurity quantities at the center of the plasma, so impurity results, to be reasonable, must be averages over some area of the plasma crossmection near the midplane. Averages over all radii are also unreasonable, because the boundary region of the plasina is not we11understood. The calculations summarized here used averages over the region $\mathbf{r} \mathbf{R}_{\mathbf{p}}$

The average electron density and temperature, assuming cylindrical symetry, are

$$
\begin{aligned}
& \bar{n}_{e}=\frac{2 \pi \Delta z \int_{0}^{R} n_{e} r d r}{2 \pi \Delta z \int_{0}^{R} r d r}, \text { and } \\
& \bar{T}_{e}=\frac{2 \pi \Delta z \int_{0}^{R} n_{e} T_{e} r d r}{2 \pi \Delta z \int_{0}^{R_{p}} n_{e} r d r}
\end{aligned}
$$

For Gaussian-shaped plasmas, the average electron density is

$$
\bar{n}_{e}=0.632 \hat{n}_{e}=\frac{0.632 \int n_{e} d 1}{\sqrt{\pi} R_{p}}
$$


To determine $\overline{\mathrm{T}}_{e}, \mathrm{~T}_{\mathrm{e}}$ was assumed to be peaked at $\mathrm{n}$ cm and to decline linearly with radius, with a slope determined using the Thoms a scattering mearurements at $0 \mathrm{~cm}$ and $6 \mathrm{~cm}$. These measurements indicate that the $T_{e}$ profile is much flatter than the density profile [5]. $\bar{T}_{e}$ was in no case smaller than $80 \%$ of $\mathrm{T}_{e}$ at $0 \mathrm{~cm}$.

Using the above averages, the impurity density, $N$, and the concentration, $N / \bar{n}_{e}$, were determined for the measured ionization states. Concentrations of unmeasured states were also estimated. They were comparable to those of the measured states. Metastable concentrations were estimated using metastble ratios determined on Alcator [32] (with large associated uncertainties), except for the important case of $0 \mathrm{~V}$. The $0 \mathrm{~V}$ metastable ratio was measured to be 2.1 (compared to 1.1 on Alcator); the difference of recomr bination processes may explain the different ratios. As the other metastable states represent a $10 \%$ contribution to the oxygen density, their metastable ratios are less important. The concentrations of helim-like states were estimated assuming a balance of charge-exchange and ionization (see Section V).

It was seen in Section IV (Table 4) that oxygen is the dominant impurity. Table C5 presents a more detailed breakdown of the concentration estimates, showing results for the data given in Table c3. As has been discussed (Table C4), emission brightnesses were measured many times. Table C5 presents results for the dates on which surveys of impurity emissions were made. The titanium concentration was estimated by assuming equal populations in Ti IV through Ti VII, excitation rate coefficients of $10^{-8} \mathrm{~cm}^{3} \mathrm{~s}^{-1}$, and brightnesses of $10^{16} \mathrm{ph} \mathrm{s}^{-1} \mathrm{~cm}^{-2} \mathrm{sr}^{-1}$. Deuteron impact excitation, which was not included in the above calculations, is a small effect (typically 10-20\%) compared to the existing uncertainties (typically 20-50\%). 
TABLE C5. DETAILED IMPURITY CONCENTRATION RESULTS

\begin{tabular}{|c|c|c|c|c|c|}
\hline \multirow[b]{2}{*}{ Species } & \multicolumn{5}{|c|}{ Concentrations (q) } \\
\hline & Measured & Calculated & Total & Result & $\begin{array}{c}\text { Uncertainty } \\
\text { Factor }\end{array}$ \\
\hline $6 / 14 / 78$ & & & & & \\
\hline $\begin{array}{l}\text { Oxygen } \\
\text { Carbon } \\
\text { Nitrogen }\end{array}$ & $\begin{array}{c}2.0 \pm 1.1 \\
0.13 \pm 0.07 \\
0.37 \pm 0.26\end{array}$ & $\begin{array}{l}1.9 \pm 1.4 \\
0.37 \pm 0.30 \\
0.43 \pm 0.39\end{array}$ & $\begin{array}{l}3.9 \pm 2.5 \\
0.49 \pm 0.37 \\
0.80 \pm 0.65\end{array}$ & $\begin{array}{l}3.2 \\
0.33 \\
0.5\end{array}$ & $\begin{array}{l}2 \\
2.6 \\
3\end{array}$ \\
\hline $\begin{array}{l}7 / 28 / 78 \\
\text { Titanium }\end{array}$ & & & & 0.3 & 4 \\
\hline $\begin{array}{r}10 / 24 / 78 \\
\text { Oxygen }\end{array}$ & $3.3 \pm 1.8$ & $3.3 \pm 2.5$ & $6.6 \pm 4.3$ & 5.3 & 2 \\
\hline $\begin{array}{r}3 / 8-9 / 78 \\
\text { 0xygen }\end{array}$ & $0.64 \pm 0.35$ & $1.7 \pm 1.4$ & $2.3 \pm 1.7$ & 1.7 & 2.4 \\
\hline $\begin{array}{c}11 / 2-3 / 78 \\
\text { Carbon } \\
\text { Nitrogen }\end{array}$ & $\begin{array}{l}0.30 \pm 0.17 \\
0.43 \pm 0.30\end{array}$ & $\begin{array}{l}0.43 \pm 0.35 \\
0.53 \pm 0.48\end{array}$ & $\begin{array}{l}0.72 \pm 0.50 \\
0.96 \pm 0.77\end{array}$ & $\begin{array}{l}0.5 \\
0.6\end{array}$ & $\begin{array}{l}2.4 \\
3\end{array}$ \\
\hline
\end{tabular}


Impurities in the streaming plasma were surveyed by firing shots without the neutral beams. Plasmas created by four guns of the deuterium-loadedtitanium-washer-stack type [51] were observed. These guns were mounted so they mapped into the midplane about $5 \mathrm{~cm}$ from the machine axis. The plasmas were quite variable and the results given here are order-of-magnitude eacimates.

The measured brightnesses are listed in Table c6, along with estimated concentrations and the plasma pararueters. Impurity concentrations were estimated using the formula

$$
\frac{N}{n_{e}}=\frac{4 \pi B}{\left.n_{e} \int n_{e} d l<\sigma v\right\rangle^{e x}}
$$

The excitation rate coefficients are shown in the table. The results in Table $\mathrm{C6}$ are within a factor of three of those obtained using a ringle plasma gun located in a different place [50]. These observations also are not iuconsistent with recent measurements of impurities deposited on a clean surface by these plasma guns [52]. However, the uncertainties in both results are large; these plasmas are not very reproducible; and the measurements of impurity deposition indicate that the spatial distribution of titanium is very uneven [52]. These plasmas have not been studied enough to be well unders tood.

Every impurity species identified during beam-fueled shots was also observed in streaming plasmas. However, the densities of impurities in the streaming plasmas are much less than the impurity densities in the beamfueled plasmas. On the basis of the experiments reported in sections $v$ and VI, it is clear that these plasma guns are not a major source of the impurities in the central 2XIIB plasma. This is not surprising, because it is 
TABLE C6. STREAMING PLASMA OBSERVATIONS

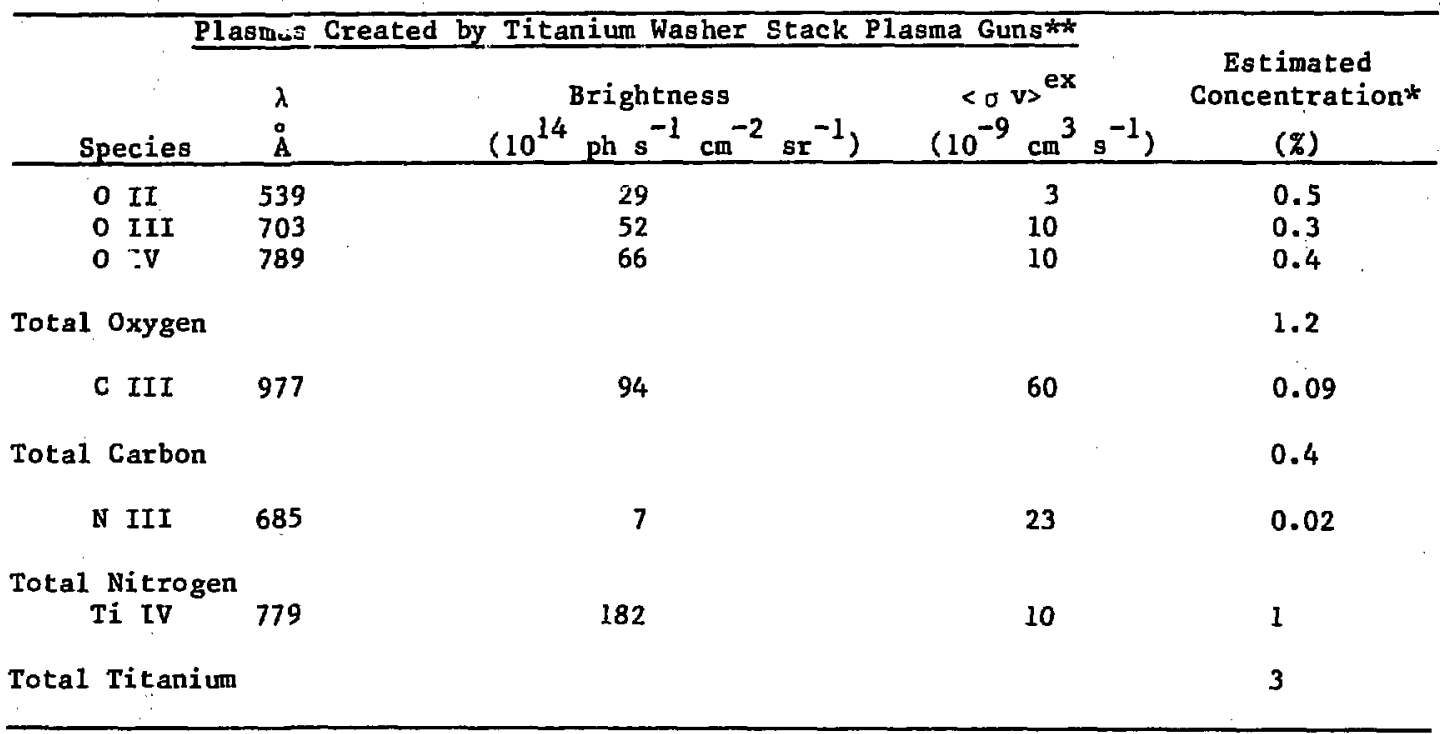

*All the oxygen is assumed to be in $0 \mathrm{II}, 0 \mathrm{III}$, and $0 \mathrm{IV}$. Other elements are assumed to be like oxygen.

$* * \mathrm{n}_{\mathrm{e}} \mathrm{dl} \sim 1.5 \times 10^{14} \mathrm{~cm}^{-2} \quad \mathrm{n}_{\mathrm{e}} \sim 1.5 \times 10^{13} \mathrm{~cm}^{-2} \quad \mathrm{~T}_{\mathrm{e}} \sim 30 \mathrm{eV}$ 
difficult for cool impurities to climb the potential hili established by the plasma and such impurities should be quickly ejected (see Section VI).

C4. Radiated Power Loss

Plasma impurities are of interest in part because they can radiate large quantities of power. The brightness measurements described above enabled calculation of the power radiated by impurities in 2XIIB. The method of calculation and the results are presented below. The radiated impurity power $108 \mathrm{~s}$ is found to be $5 \%$ of the deposited neutral beam power. This low percentage is due to the high density of energy deposition produced by neutral beam heating in this machine.

It must be emphasized that these results apply only to the impurities near the midplane. The regions outside the central plasma contain several times as many particles, and may have a higher concentration of impurities, producing a much larger radiation loss. These power losses were not measured during this study. However, they are probably not dominant, because, within a factor of 2 , the deposited power has been measured to flow out the ends of 2XIIB [ 5$]$.

Theory of Spectroscopic Power Loss Measurements

Spectroscopy provides an indirect measurement of the power loss due to line radiation. The determination of this power loss from absolute brightnesses is described in this section. A more extensive discussion may be found in Ref. [53]. Bolometric measurements are a desirable check on the completeness of these calculations, but such measurements were not made on 2XIIB. Soft $x$-ray measurements show power losses consistent with those expected from oxygen transitions near $60 \mathrm{eV}$ [54]. 
There is no universal relation between power $108 s$ and brightness. The measured brightness results from an integration of light emitted along a line of sight through the plasma:

$$
B=\frac{1}{4 \pi} \int E(\Sigma) d 1
$$

Power loss by contrast results from the emission of light throughout the plasma volume:

$$
P_{L}=h v \int E(r) d v
$$

$P_{L}$ is the power radiated from the plasma by a spectral line with energy hv. Once the spatial variation of $E(r)$ is known one can relate power loss directly to brightness. Assuming cylindrical symetry and a plasma of length $I$, one obtains

$$
\begin{aligned}
& B=\frac{1}{2 \pi} \int_{0}^{\infty} E(r) d r, \text { and } \\
& P=2 \pi h \nu L \int_{0}^{-} E(r) r d r .
\end{aligned}
$$

Spatial scans of 2 XIIB show that the radial profile of $E(r)$ is approximately Gaussian. Taking $\mathrm{E}(\mathrm{r})=\mathrm{Ae}^{-\mathrm{r}^{2} / \mathrm{a}^{2}}$ one obtains:

$$
\begin{aligned}
& B=\frac{a}{4 \sqrt{\pi}} A \text { and } \\
& P=h \pi \mathrm{Lva}^{2} \mathrm{~A}, \quad=.
\end{aligned}
$$

Hence

$$
P=4 \pi^{3 / 2} \text { hvL } \text { a B }
$$


This gives the relation of power loss to measured brightness; assuming cylindrical symmetry and a Gaussian emission profile.

The total radiated power is desired, but Eq. (c11) gives only the power radiated by an observed $1 \mathrm{ine}$. It is not practical to observe al radiating lines, so a calculation is necessary in order to-relate the total power loss to the measured brightnesses. This relation is developed below.

As $E(r)$ is proportional to $\langle\sigma v\rangle^{e x}$, the rate coefficient for electron impact excitation of the transitions, one has, for the power radiated by any given line, 'P,

$$
\mathrm{P}_{\mathrm{L}} \propto \frac{\operatorname{covs}_{\mathrm{L}}^{\mathrm{ex}}}{\lambda_{\mathrm{L}}}
$$

The relation of this power to the power radiated by all lines of an ionization state, $P_{S}$, is

$$
\mathbf{P}_{\mathbf{S}}=\mathbf{F} \mathbf{P}_{\mathrm{L}}
$$

in which

$$
F=\left[\sum_{i} \frac{\lambda_{L}}{\lambda_{i}} \frac{\langle\sigma v\rangle_{i}^{e x}}{\left\langle\sigma_{v}\right\rangle_{L}^{e x}}+R_{m} \sum_{j} \frac{\lambda_{L}}{\lambda_{j}} \frac{\langle\sigma v\rangle_{j}^{e x}}{\langle\sigma v\rangle_{L}^{e x}}\right]
$$

The first sum is taken over the significant ground state transitions; and the second expression is summed over the important metastable transitions. The best values of $\left\langle\sigma^{\circ}\right\rangle$ exist for the transitions to the lowest excited states. The ratios of the other rate coefficients to these we 11 known ones (i.e., <ov $>$ ) were estimated using staudard formulas [55] and the oscillator strengths of wiese et al. [56]. $R_{m}$ is the ratio of mestastable 
population to ground state population. By including all relevant proportionalities, F converts the power radiated by the line into that radiated by the entire ionization state. Other ionization states may be included in $F$ in the same way the metastable system is in Eq. (C13), but this is undesirable; in this study it was only necessary in the unimportant case of $N$ IV.

The radiated power loss due to an entire species is then given by

$$
P_{\text {SPECIES }}=\sum P_{S}
$$

These uncertainties in these calculations have several sources. The absolute calibration of the spectrometer, the measurement techniques, the model relating radiated power to brightness, and the power factor $F$ all contribute to the uncertainty. However, as the relative populations of various ionization states need not be considered, this method is considerably more accurate than a purely a priori computation.

\section{Radiated Power Loss Results}

The power loss due to impurity radiation was calculated as described above. Brightness measurements have been made for all important ionization states of oxygen and for the significant states of carbon and nitrogen. All $\Delta \mathrm{n}=0$ anci $\Delta_{\mathrm{n}}=1$ transitions were considered in evaluating the power factor F. Metastable ratios were taken from measurements performed on Alcator, except for the important case of $0 \mathrm{~V}$, which was checked in the 2 XIIB plasma. The results of these calculations are shown in Table C7.

Most of the power radiated by 2 XIIB impurities is from $0 \mathrm{~V}$ and $0 \mathrm{VI}$. This is due to the dominance of oxygen and to the fact that nitrogen and carbon reach helium-like states at lower temperatures than oxygen does. 
TABLE C7. LIGHT IMPURITY RADIATED POWER : LOSS

\begin{tabular}{lcc}
\hline Species & $\begin{array}{c}\text { Power Radiated } \\
\text { By Measured } \\
\text { Lines } \\
(\mathrm{kW})\end{array}$ & $\begin{array}{c}\text { Total Power } \\
\text { Radiated by } \\
\text { Species } \\
(\mathrm{kW})\end{array}$ \\
\hline $\begin{array}{l}\text { 6/14/78 } \\
\text { 0xygen } \\
\text { Nitrogen } \\
\text { Carbon }\end{array}$ & 11.1 & $31 \pm 15$ \\
$\begin{array}{l}\text { ToTAL } \\
\text { 10/24/77 }\end{array}$ & 1.8 & $6.4 \pm 5.7$ \\
Oxygen & 1.3 & $1.7 \pm 0.9$ \\
\end{tabular}

Titanium radiation power loss is not included in these calculations. (A crude estimate based on a $0.3 \%$ concentration and the calculations of Post et al. [40] is $3.5 \mathrm{~kW}$ ).

Impurities also remove power from the plasma as a result of ionization, which costs energy and produces cold electrons. This power loss depends on the impurity currents and maximum ionization states. It is estimated to be quite small ( $\sim_{3} \mathrm{~kW}$ ) for carbon, nitrogen, andtitanium. The effects of oxygen on the 2XIIB power balance were discussed in Section $V$.

Apparently, the impurity radiation from the central 2XIIB plasma did not play a major role in determining its parameters. Neither $T_{e}$ nor the $T_{e}$ radial profile was significantly affected by power losses such as these. The electron temperature limit due to impurity power loss may be estimated by equating this power loss to the Spitzer drag power input to the electrons from the hot ions. The radiated power loss determined above allows a maximum 
2 XIIB electron temperature of about $300 \mathrm{eV}$ on $6 / 14 / 78$, which is much higher than the observed values $\left(T_{e} \approx 60 \mathrm{eV}\right)$. Impurity radiation could be a significant power loss mechanism in 2 XIIB only if this was primarily due to impurities in the end fans--and the end fans were not observed in this study. 
This appendir- contains the spatial data from 2XIIB which was not included elsewhere. A brief summary of this data is given below. The other spatial scans may be found in Figs. 7, 9, and C5.

Figures D1 and D2 show the N III $685 \AA$ data and the Ti IV $779 \AA$ which justify the use of the constant-density model for these elements. The nitrogen density profile can be made shell-like or peaked depending on the curve drawn through the data; the flat profile is in between. The format of this figure is the same as that of Fig. C5, discussed in Appendix C. The titanium data suggests a slightly peaked spatial profile--its scale length is less than that of the line density. But, as the titanium profile is broader than the profile of electron density squared, the constant density model is as good a choice as the constant concentration model.

Some additional o V $630 \AA$ spatial data is shown in Fig. D3. The broad distribution and symmetric profile may be observed. The symmetry and essential structure of 0 II $539 \AA$ A emissions is shown in Figs. D4 and D5. If there was a dominant 0 II she11, the 0 II profile would be broader than the line density profile; it is not. Fina1ly, Fig. D6 shows some additional C III 977 A data, which supports use of the constant-density model for carbon. 
4/27/78 Shots 21-32

Peak N $111685 \AA \mathrm{B}=1.5 \times 10^{15} \mathrm{ph} \mathrm{s}^{-1} \mathrm{~cm}^{-2}$

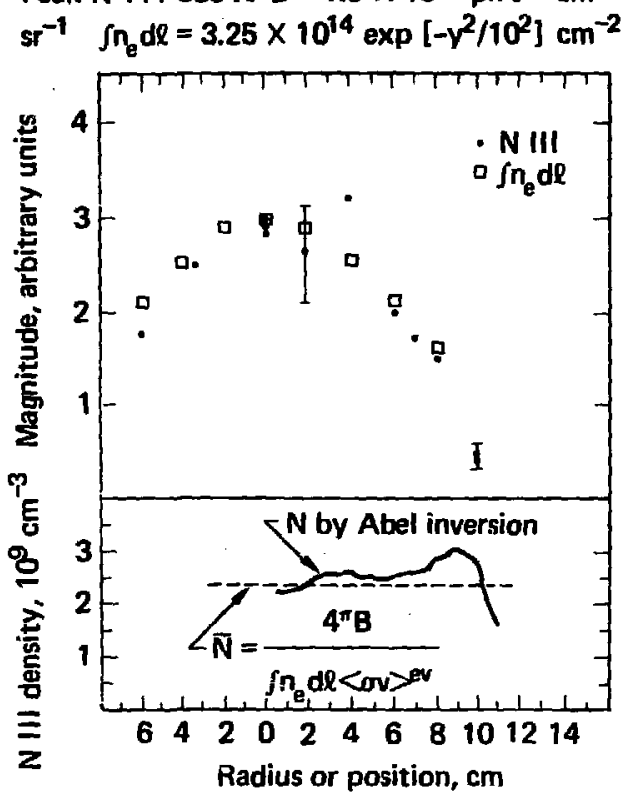

Figure. D1. Spatial scan of N III 685 A. 


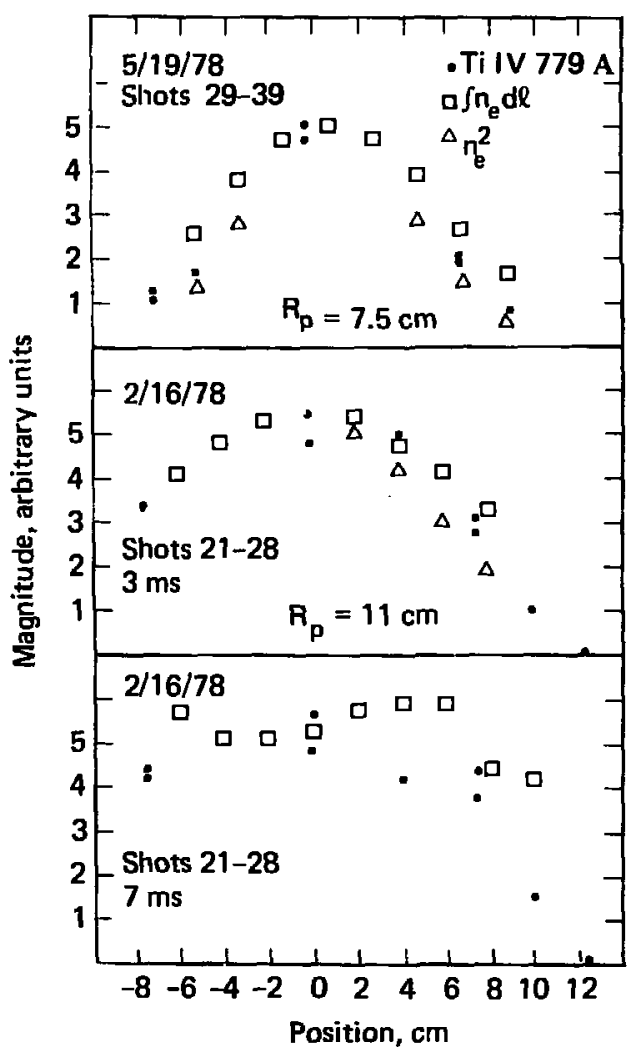

Figure D2. Spatial scans of Ti IV $779 \stackrel{\circ}{\mathrm{A}}$ 


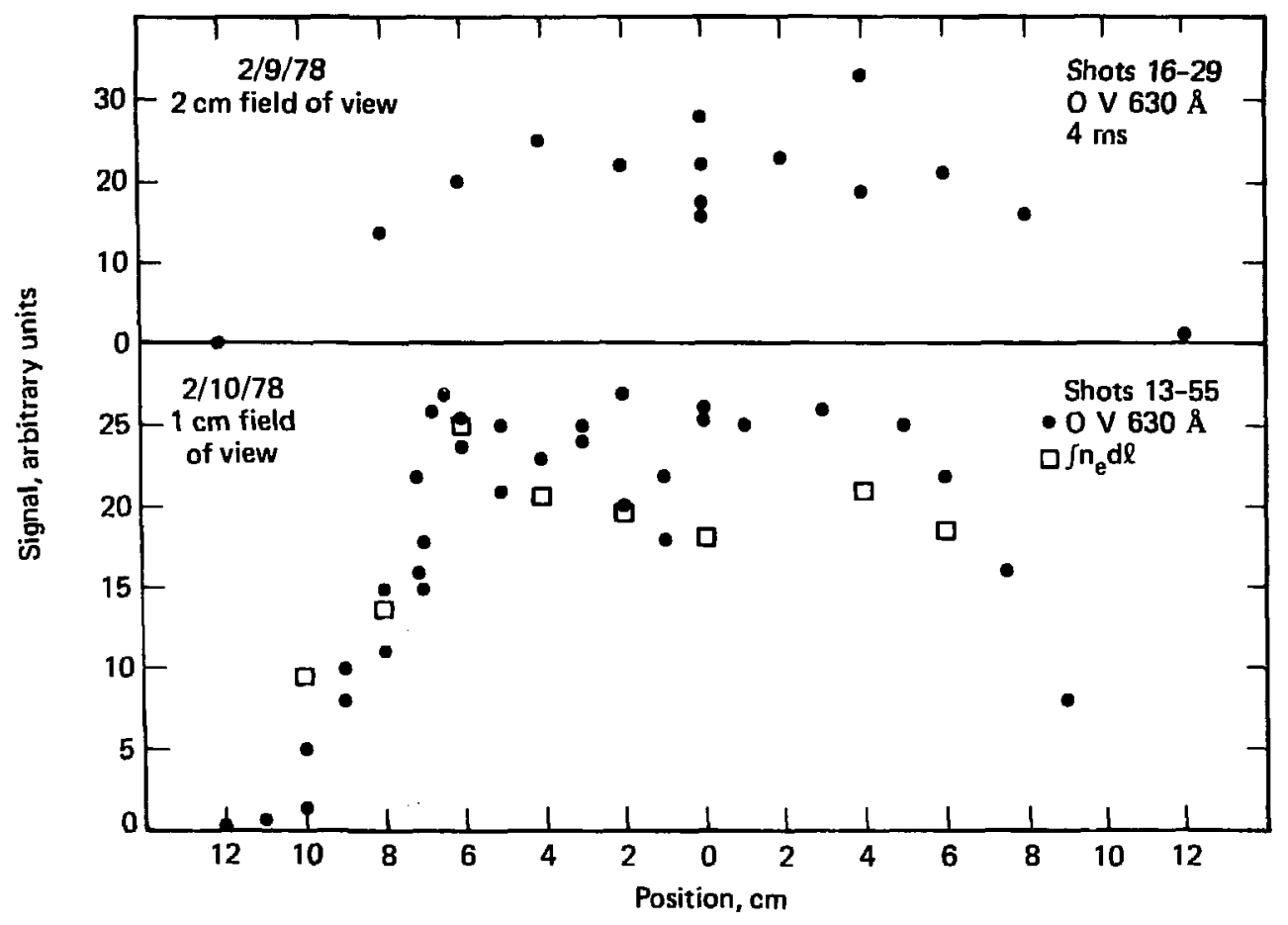

Figure D3. Spatial scans of o V $630 \AA$ A. 


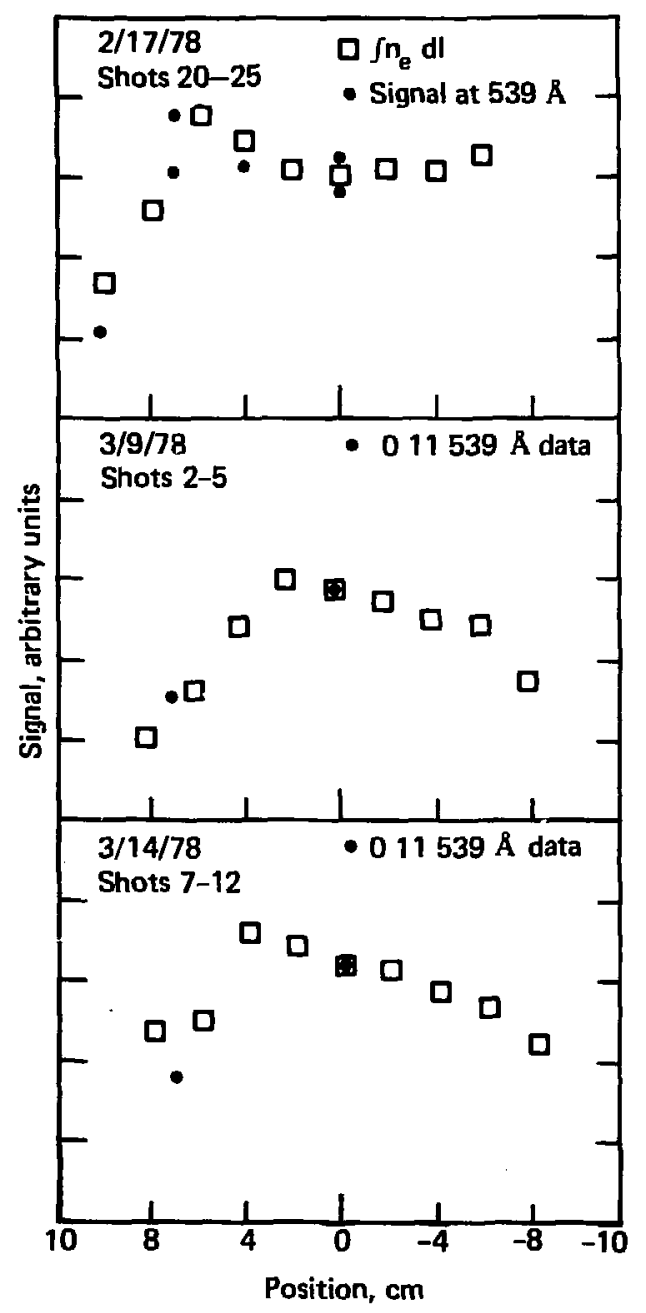

Figure D4. Spatial scans of 0 II $539 \AA$. 


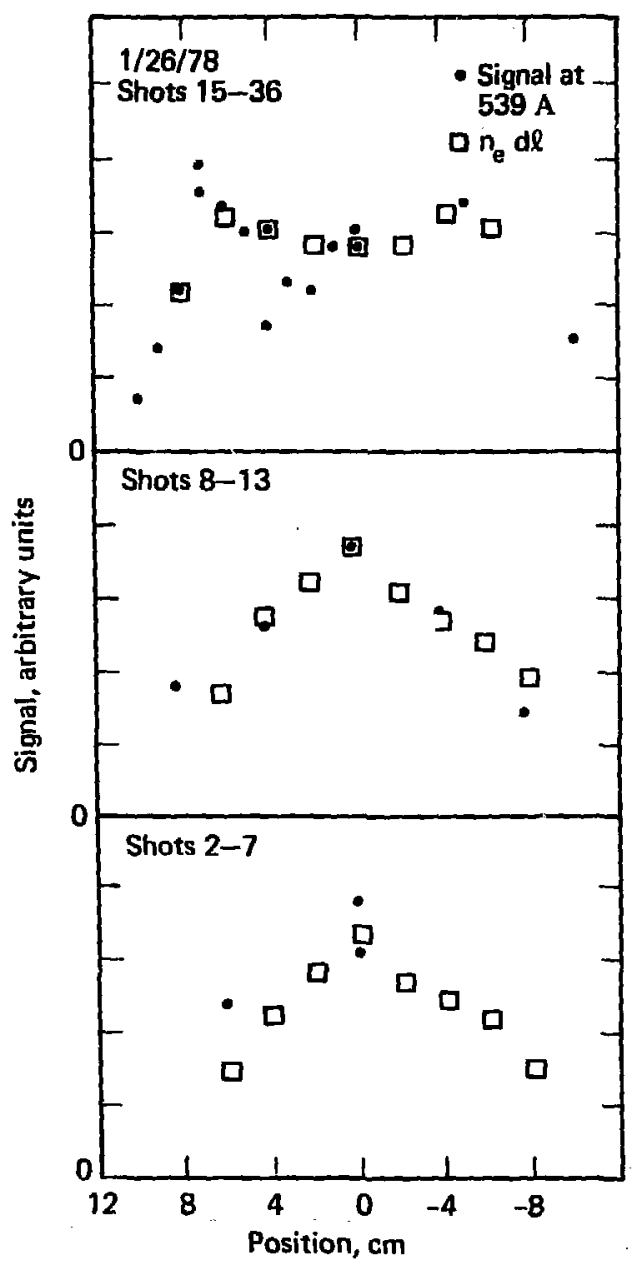

Figure D5. Spatial scans of 0 II $539 \AA$. 


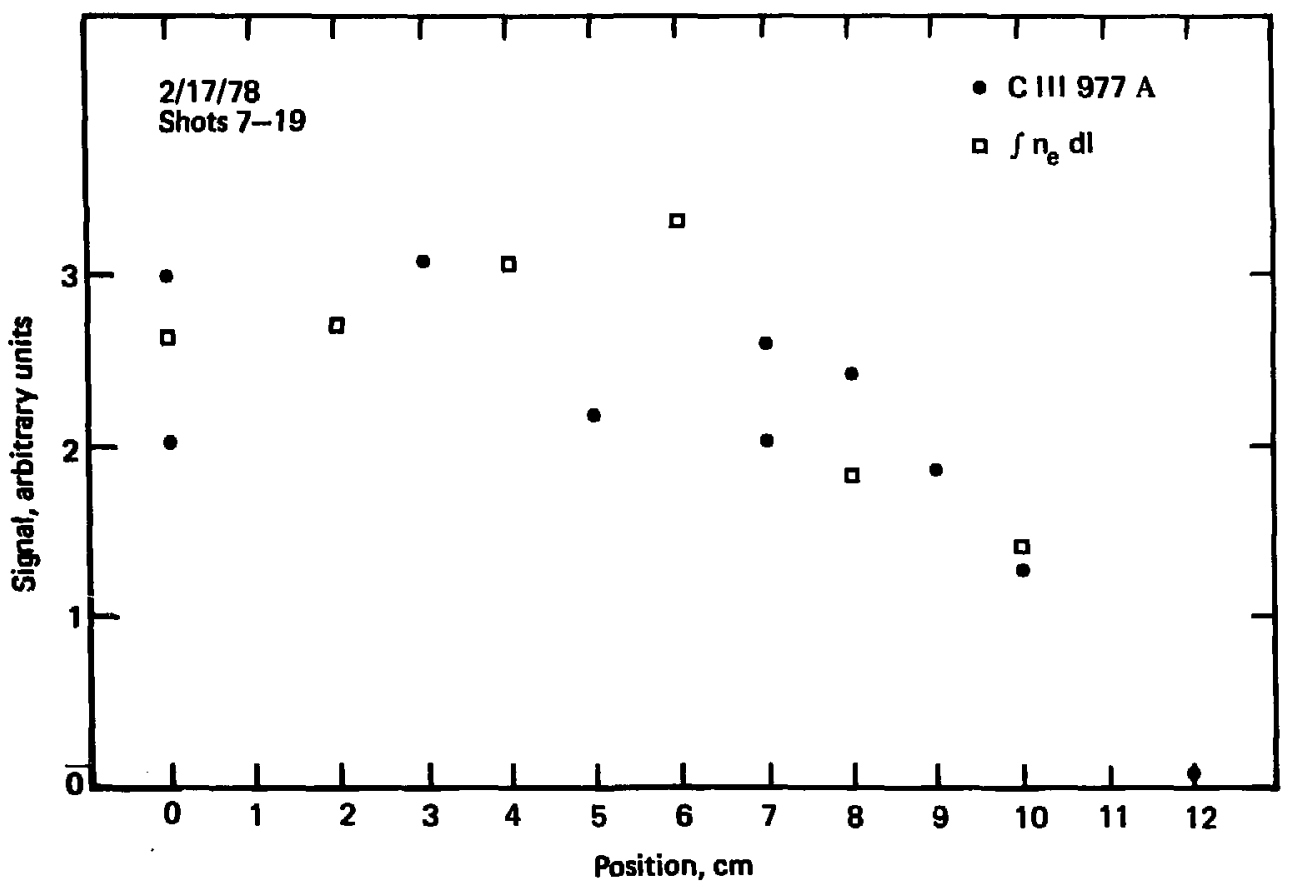

Figure D6. Spatial scan of C III $977 \AA$ 


\section{APPENDIX E. A MODEL OF BEAM INJECTION OF OXYGEN}

The model of beam-injected-oxygen emission anplied in Fig. 8 is derived in this Appendix. Both the scaling and magnitude of the observed data are predicted, given the known dependencies of electron-line density and deuterium neutral density on beam current. The essential physics of the model is that the 0 II brightness depends not only upon the concentration of oxygen in the beams, but also upon the relative attenuation of oxygen and deuterium by the plasma.

The 0 II brightness is

$$
B=\frac{1}{4 \pi} \int n_{e} d l\left\langle\sigma^{v}\right\rangle^{e x} \overline{n_{0}} \text { II },
$$

as discussed in Section III. The line density scaling is

$$
\int n_{e} d 1=1.5 \times 10^{12} I_{b}^{1.1}(A)
$$

for the data under consideration. The mean 0 II density due to beam-injected oxygen (assumed throughout this appendix) can be calculated as follows.

The $O$ II concentration is determined by the oxygen and deuterium beam currents, attenuation, and particle lifetimes. We have, in general

$$
I(1-T)=\int \frac{N}{\tau} d V
$$

Here the particle input is beam current, I, times attenuation (= 1-transmission, $T$ ). The particle outflow is density, divided by lifetime, integrated over volume. For 0 II, the particle lifetime is determined by ionization, $\tau=\left(n_{e}<\sigma_{v}>^{i o n}\right)^{-1}$, so 


$$
\begin{aligned}
\int \frac{n_{0} I I}{\tau} d V=\int n_{0} I I n_{e} \operatorname{cov}>^{i o n} d V & \\
& =\overline{n_{0}} \text { II }\left\langle\sigma v>^{i o n} \int n_{e} d V\right.
\end{aligned}
$$

Similarly, the lifetime of deuterium particles is determ'ned by chargeexcharige, $\tau=\left(n_{D}<\sigma_{v}>^{c x}\right)^{-1}$. The charge-exchange lifetime is typically $200 \mathrm{Hs}$, much shorter than that of any other process. This gives

$$
\begin{aligned}
& \int \frac{D^{+}}{\tau} d V=\int{ }_{D}+{ }_{D}^{n}\langle\sigma v>C x d V \\
& ={\overline{n_{D}}}^{<\sigma_{v}}>^{c x} \int n_{e} d v
\end{aligned}
$$

Now, after defining $f$, the fraction of oxygen in the beams, as

$$
f_{0}=\frac{I_{0}}{I_{D}}=\frac{I_{0}}{I_{b}}
$$

one can use Eqs. (E3), (E4), and (E5) to obtain

$$
f_{0}\left(\frac{1-T_{0}}{1-T_{D}}\right)=\frac{\overline{n_{0} I I}\langle\sigma v\rangle^{\text {ion }}}{\bar{n}_{D}\langle\sigma v>c x}
$$

From this

$$
\overline{n_{0} I I}=E_{0}\left(\frac{1-T_{0}}{1-T_{D}}\right) \frac{\langle\sigma v\rangle_{\langle\sigma v\rangle^{i o n}}^{c x}}{n_{D}}
$$

The scaling of $\overline{n_{D}}$ for the scan of Fig. 8 is found in Appendix $B$ to be

$$
\overline{n_{D}}=2.1 \times 10^{8} \mathrm{I}_{\mathrm{b}}^{0.85}
$$


We now have for the brightness

$$
\vec{B}=\frac{f_{0}}{4 \pi}\left(\frac{1-T_{0}}{1-T_{D}}\right) \frac{\langle\sigma v\rangle^{c x}\langle\sigma v\rangle^{e x}}{\langle\sigma v\rangle^{i o n}} \int n_{e} d l \overline{n_{D}}
$$

If one takes $\left\langle\sigma_{v}\right\rangle^{c x}=1.3 \times 10^{-7},\langle\sigma v\rangle^{e x}=4.1 \times 10^{-9}$, and

$<\sigma \mathrm{v}>^{\text {ion }}=1.4 \times 10^{-8}$, which are appropriate for this data, and use the scalings of Eqs. (E2) and (E7), the result is

$$
B=9.5 \times 10^{11} \mathrm{f}_{0}\left(\frac{1-\mathrm{T}_{0}}{1-\mathrm{T}_{\mathrm{D}}}\right) \mathrm{I}_{\mathrm{b}}^{1.95} \equiv 9.5 \times 10^{11} \mathrm{f}_{0} \mathrm{~F}\left(\mathrm{I}_{\mathrm{b}}\right)
$$

The evaluation of $F\left(I_{b}\right)$ requires knowing $T_{0}$ and $T_{D}$. The transmission of a species through a plasma is given by

$$
T=\exp \left[-\int n_{e}(\Sigma<\sigma v>) \frac{1}{v} d 1\right]
$$

An analysis of deuterium transmission by W. C. Turner (which led to the successful beam attenuation density diagnostic), coupled with the results of a computer code he wrote to calculate transmission of typical 2 XIIB beams through typical 2 XIIB plasmas, indicates that the average transmission of deuterium is

$$
\mathrm{T}_{\mathrm{D}}=\exp \left[-\int \mathrm{n}_{\mathrm{e}} \mathrm{dl} \sigma_{\mathrm{D}_{\mathrm{eff}}} / 1.41\right]
$$

in which $\sigma_{D_{\text {eff }}}=1.54 \times 10^{-15} \mathrm{~cm}^{2}$. For oxygen one takes

$$
\sigma_{0_{\text {fff }}}=\frac{\left\langle\sigma_{\mathrm{v}}\right\rangle \text { ion }+\langle\sigma v\rangle^{\text {ion }}+\langle\sigma v\rangle^{\mathrm{cx}}}{\mathrm{D}^{+}}
$$

and substitute $\langle\sigma v\rangle_{e}^{\text {ion }}=7 \times 10^{-8},\left\langle\sigma v>_{D}^{\text {ion }}=2 \times 10^{-8},<\sigma v\right\rangle_{D}^{c x}=10^{-7}$, and $v=4.4 \times 10^{7}(=16 \mathrm{keV})$ to obtain $\sigma_{0_{\text {eff }}}=4.32 \times 10^{-15}$. This value is used in Eq. (11) to calculate T. (Double ionization, a 15\% effect, is neglected for this factor-of-2 model.) 
By using these values and computing the attenuation, the scaling of the relative attenuation is found to be

$$
\frac{1-T_{0}}{1-T_{D}}=15.7 I_{b}^{-0.4}
$$

This is a good fit because this plasma is neither optically thin nor optical1y thick to penetration by the high-energy-beam atoms. The final equation for $B$ is:

$$
B=1.5 \times 10^{13} \mathrm{~F}_{0} \mathrm{I}_{\mathrm{b}}^{1.55}
$$

The predictions of the model are shown in Fig. 8 . It is seen that the scaling of the model fits the data, and that the predicted brightness is consistent with the data (considering the factor-of-2 nat:re of this calculation). 
APPENDIX F. POSSIBLE SOURCES OF CARBON, TITANIUM, AND NITROGEN

F1. Introduction

This Appendix reviews the possible sources of impurities other than oxygen in 2XIIB. After the initial measurements of EUV emissions, oxygen studies were emphasized. The oxygen results were discussed in section V. Other impurities were studied with less emphasis, but some significant data were obtained. The most is known about carbon, which will be considered first. Significantly, it has become clear that the streaming-plasma guns were not the primary source of carbon or titanium. Wall-sputtering of titanium and methane are believed to be the source of these impurities, but this has not been demonstrated; and certain important questions about heating and transport remain unanswered. Another possible source of carbon and titanium is cathode-spotting at the end walls.

\section{F2. Carbon Studies}

In this section, the EUV carbon data from 2 XIIB is reviewed and the most likely carbon source is determined. The carbon observed was apparently introduced axially, and had a temperature of a few keV; but the streaming guns were not the only significant source. The methane produced during gettering might have provided a large carbon current, a small fraction of which was heated and penetrated the central plasma.

\section{A Review of the Carbon Data}

The following data on carbon emissions was obtained: the variation of C III $977 \AA$ with beam current (6/9/78); a good spatial scan (2/16/78) and a good spectral scan (2/15/78), with supporting scans on other days for each; the observation of cleanup; and carbon emissions with various stabilizing 
techniques (stream guns, gas box, and e-beam). In this subsection: the data are reviewed; possible carbon sources are considered below. The reader may wish to skip this subsection and refer to it as indicated below.

\section{(i) The Beam Current Scan}

C III $977 \AA$ data obtained as the neutral beam current was systematically varied is shown in Fig. 8. This beam current scan was performed June 9, 1978 with 25 torr pressure in the gas box injectors. A least-squares fit indicates that the C III emissions rose as the 0.65 power of beam current. The electron-line density was proportional to beam current, except at very low beam currents. The C III emissions rose as the 0.54 power of $I_{b}$ if these low current shots are eliminated. As the brightness is proportional to the C III density times the line density, it appears that the total amount of C III in the plasma declined as beam current increased.

The above results place limitations on the possible sources of carbon. Axial carbon sources are allowed, as the C III will be more attenuated when the axial line density increases. Sources of well trapped highly ionized carbon are also allowed. However, hypothetical carbon sources which introduce increasing amounts of neutral carbon at the midplane as a function of beam current are not consistent with this data, unless that carbon returns to the midplane after being heated.

\section{(ii) C III Spatial Data}

Figure C5 shows the C III spatial data taken February 16, 1978, as well as the density profile obtained by Abel inversion. While some shell structure of central peaking is not ruled out by the curves which can be drawn through the data, there was clearly C III near the center of 2 XIIB. 
The existence of twice ionized carbon in this location is evidence against low energy sources of carbon with a charge $\leq 2$. The carbon III got to the center of the plasma either through penetration, requiring a high-energy source, or through charge-exchange from $I V$, requiring trapped carbon ions.

\section{(iii) The Spectral Profile of C III $977^{\circ}$}

The data clearly show that the C III $977 \AA$ line becomes broad in the course of a shot. Figure C2 presents a C III $977 \AA$ spectral scan; the observed broadening corresponds to a mean energy of $4 \mathrm{keV}(+40 \%)$. This requires that the carbon was heated either before or after injection. Beam injected carbon would have to enter the plasma as $\mathrm{CO}$ or $\mathrm{CO}_{2}$ (rather than $\mathrm{C}$ or $\mathrm{CH}$ ) to explain the observed energy. There are other possible heating mechanisms, some of which will be discussed below. It is worth remembering that 6-keV carbon was also observed from 2XII, which had no neutral beams [3]. Also note that additional cold carbon may have penetrated the plasma when the gas box was used, as a result of the modification of the plasma potential.

iiv) The Observed Cleanup of Carbon and Carbon

Versus Stabilizing Method

During May, June, and July, 1978, carbon emissions were routinely monitored. It was observed that the C III $977^{\circ}$ emissions decreased as shots were fired after each evacuation of 2 XIIB. In addition, several different sources of stabilizing streaming plasma were used (see Section II). No stabilization technique produced beam-fueled plasmas which had substantially less carbon than any other. Figure Fl is a plot of C III $977 \AA$ emissions as a function of shots after pump-down. Shots taken during the same evacuation using similar control settings are connected by lines. 


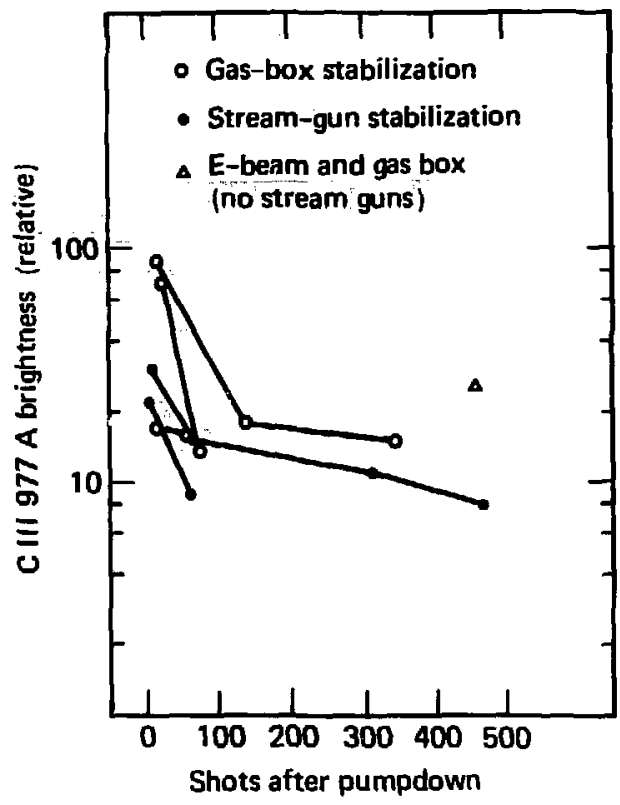

Figure F1. C III $977 \AA$ A emissions declined during the first few shots after $2 X I I B$ was evacuated.

This data is compiled in Table Fl, along with the electron density and line average carbon density.

These results show that the decline of carbon emissions represented a decrease in the amount of C III in the plasma. The total carbon concentration also probably declined, because the fraction of carbon III should be constant, unless $T_{e}$ changes, and $T_{e}$ was fairly constant throughout these runs .

Two aspects of this data are important. First, the carbon "cleaned up" bui did not decline by orders of magnitude. Second, there were similar quantities of carbon present (after cleanup), whether or not any given stabilizing source was in use. The implications of this are discussed below.

\section{An Evaluation of Possible Carbon Sources}

The potential carbon sources may be evaluated by use of the data presented above. This subsection argues that an ionized energetic axial flux is consistent with the EUV data, but' that the carbon did not come only 
TABLE Fl. CARBON CLEANUP DATA

\begin{tabular}{|c|c|c|c|c|c|c|}
\hline Date & Shot & $\begin{array}{c}\text { Shot } \\
\text { After } \\
\text { Pump Down }\end{array}$ & $\begin{array}{l}\int \mathrm{n}_{\text {ed } 1} \\
\left(10^{14}\right)\end{array}$ & $\begin{array}{c}\text { C III } \\
977 \AA \\
\text { B } \\
\left(10^{15}\right)\end{array}$ & $\overline{\mathbf{N}}$ & Machine Conditions \\
\hline $\begin{array}{l}5 / 19 / 78 \\
5 / 21 / 78\end{array}$ & $\begin{array}{l}18 \\
16\end{array}$ & $\begin{array}{l}26 \\
82\end{array}$ & $\begin{array}{l}3.7 \\
7.5\end{array}$ & $\begin{array}{l}70 \\
14\end{array}$ & $\begin{array}{l}3.7 \\
3.6\end{array}$ & 100 torr in gas box \\
\hline $\begin{array}{l}6 / 1 / 78 \\
6 / 6 / 78 \\
6 / 22 / 78 \\
6 / 28 / 78\end{array}$ & $\begin{array}{r}10 \\
19 \\
8 \\
38\end{array}$ & $\begin{array}{r}10 \\
75 \\
309 \\
468\end{array}$ & $\begin{array}{l}9.7 \\
10 \\
9 \\
10\end{array}$ & $\begin{array}{r}32 \\
15 \\
11 \\
8\end{array}$ & $\begin{array}{l}6.4 \\
2.9 \\
2.4 \\
1.5\end{array}$ & $\begin{array}{c}\text { Stream guns only } \\
" 1 \\
"\end{array}$ \\
\hline $\begin{array}{l}6 / 1 / 78 \\
5 / 9 / 78 \\
6 / 22 / 78\end{array}$ & $\begin{array}{l}17 \\
18 \\
42\end{array}$ & $\begin{array}{r}17 \\
137 \\
343\end{array}$ & $\begin{array}{r}13 \\
9.5 \\
11\end{array}$ & $\begin{array}{l}87 \\
18 \\
15\end{array}$ & $\begin{array}{r}12.9 \\
3.7 \\
2.6\end{array}$ & $\begin{array}{c}100 \text { torr in gas box } \\
" 1\end{array}$ \\
\hline $6 / 28 / 78$ & 29 & 459 & 9 & 26 & 5.6 & $\begin{array}{l}100 \text { torr gas plus e-beam } \\
\text { No stream guns used }\end{array}$ \\
\hline $\begin{array}{l}7 ; 14 / 78 \\
7 / 15 / 78\end{array}$ & $\begin{array}{r}7 \\
26\end{array}$ & $\begin{array}{r}7 \\
64\end{array}$ & $\begin{array}{l}8.5 \\
11\end{array}$ & $\begin{array}{r}22 \\
9\end{array}$ & $\begin{array}{l}5.0 \\
1.6\end{array}$ & Stream guns only \\
\hline $\begin{array}{l}7 / 14 / 78 \\
7 / 15 / 78\end{array}$ & $\begin{array}{l}20 \\
22\end{array}$ & $\begin{array}{l}20 \\
60\end{array}$ & $\begin{array}{l}17 \\
17\end{array}$ & $\begin{array}{l}18 \\
17\end{array}$ & $\begin{array}{l}2.0 \\
1.9\end{array}$ & 400 torr in gas box \\
\hline
\end{tabular}


from the stream guns. The neutral beams and low-energy radial fluxes are rejected as direct sources.

\section{(i) Ionized Energetic Axial Sources}

Suppose there is a source of high-energy carbon between the midplane and the end wa11 in 2XIIB. These ions can penetrate the plasma, but will be further ionized as they do. When the axial line density increases as the beam cury nt is raised, the ionization rate will increase. The fraction of low-Z carbon ions reaching the midplane will in consequence decrease. This uleans the C III concentration may decrease as beam current is raised, as was observed.

As the radial position of such carbon when it reaches the midplane will be determined by the magnetic field line it follows in the fan, there is no reason to expect an absence of $C$ III in the center of the 2XIIB plasma, so long as the carbon relocity is high enough that it can penetrate without being further ionized. Thus, the observation of C III throughout the central plasma was consistent with such a source.

Finally, such high energy carbon would produce some Doppler broadened emissions, and some of it would become mirror trapped as a result of collisions, leading to a population of mirror-confined energetic carbon. As a result, $6 \mathrm{keV}$ carbon could be observed in 2 XIIB.

The observations of cleanup of carbon, and of the effect of stabilizing techniques on carbon emissions, do not bear on this hypothesis. The existence of a high energy axial flux of carbon ions is thus consistent with the EUV data. 


\section{(ii) The Stream Guns}

The streaming plasma guns cannot be the primary source of carbon. If the stream guns were the primary carbon source, their use would noticeably affect the amount of carbon in the plasma. Whether stream guns alone, stream guns with gas box, gas box after stream guns, or gas box with e-beam were used to stabilize the plasma did not appear to affect the carbon concentration. It follows that the stream guns were not the primary carbon source, although they may have contributed to the "carbon pool" in the end fan.

\section{(iii) The Neutral Beams}

The neutral beam current scan weighs heavily against beam-introduced carbon. The assumptions which apply to beam-injected O II also apply to C III and the model introduccd above (Appendix E) can be applied to carbon. For the C III brightness:

$$
B \propto\left(\frac{1-T_{c}}{1-T_{D}}\right) I_{b}^{2} \text {. }
$$

The weakest dependence of C III emissions on beam current is obtained if the carbon was in a heavy molecule which is entirely trapped $\left(T_{c} \sim 0\right)$, and one uses the thin plasma limit for deuterium (this is a fair approximation for the $6 / 9 / 78$ scan $)$. In this case $\left(1-T_{b}\right) \propto I_{b}$ and one obtains

$$
B \propto I_{b}
$$

The observed dependence of C III $977^{\circ}$ on beam current was not this strong. The carbon is apparently not injected by the neutral beams. 


\section{(iv) Radial Low Energy Carbon}

The data presented above also makes low energy radial carbon fluxes very unlikely as a primary source of carbon. The low-energy carbon should be swept out by the plasma potential before it can be heated to the energies which were observed by Doppler broadening. Low-energy carbon should also be ejected before it can diffuse inward and become distributed throughout the plasma, yet the carbon in 2 XIIB was observed to be widely distributed.

In addition, radial fluxes would be expected to result from sputtering, and this hypothesis conflicts with the data from the beam current scan. The total charge-exchange flux leaving the plasma rises as almost the square of the beam current (being proportional to the neutral density times the electron density times the fraction of charge-exchange neutrals which escape the plasma). The total high energy neutral flux striking walls and beam dumps, including untrapped beam current, rises less rapidly but still depends on the beam current to a power greater than 1. The carbon III emissions, and hence the neutral carbon flux, depended on the beam current to a power less than 1; this indicates that the primary direct source of carbon was not a sputtered radial flux.

It is possible that low energy radial carbon fluxes contributed indirectly to the carbon concentration in the center of 2 XIIB. These fluxes may have been ionized at the plasma boundary, and swept away from the midplane by the plasma potential. Later, some of this carbon may have returned by some mechanism to the central plasma.

\section{A Plausible Source of Carbon}

As the data is most consistent with a high-energy axial source of carbon, one must ask whether such a source could arise by known mechanisms. In this section the following tentative hypothesis is considered. Carbon, 
wnich orıginates as methane deposited on the walls during gettering, is heated, and penetrates the central plasma. 'The carbon observed at the midplane is partly this high energy untrapped flux, and is partly carbon of the same origin which has become mirror trapped.

Once a few shots were taken after pumping down, carbon emisaions varied little despite significant changes in 2 XIIB operation. This leads one to look for carbon source which was always present. The obvious choice is the titanium gettered walls of the machine. As the titanium wire contains some carbon impurity, methane $\left(\mathrm{CH}_{4}\right)$ is produced during gettering (this is observed with the residual gas analyzer), and some of the methane will adhere to the wall. This methane can then be removed from the wall during a shot, by means of sputtering, cathode arcing, or some other mechanism.

The carbon, introduced as methane, must be heated to penetrate the central plasma. Heating of impurities in these plasmas is poorly understood, but several mechanisms are possible. Partially ionized carbon may be resonantly heated by the dominant deuterium ion cyclotron radiation in certain regions of magnetic field. Nonresonant rf heating is possible, based on past experience with 2XII. Carbon may diffuse to high enough energies while trapped between a stream gun and the central plasma. Carbon which gains $z \phi(z=3$ to 4$)$ when it is ejected may need only $\phi$ or $2 \phi$ of energy to penetrate the plasma after backscattering at the end wall. None of these processes is likely. Further study might reveal whether in combination they can lead to the observed carbon concentration. Simple calculations suggest that roughly 20-equiv A of 2-keV carbon atoms could praduce the observed carbon concentration, as a result of trapping by coulomb scattering. 
Some experiments could be performed to investigate impurity penetration as described above. One could introduce an impurity between the centra1 plasma and the mirror and monitor $i t s$ presence in the end fan plasma and at the midplane. If the argument above is correct, a small quantity of the impurity would appear near the midplane with high energy. A more subtle experiment would be to install some getter wires of an unusual substance throughout the machine and to investigate the characteristics of that substance in the plasma.

\section{F3. Titanium and Nitrogen}

This section sunmarizes what little is known about titanium and nitrogen sources. Neither of these elements was thoroughly studied, but the initial, tentative results deserve a brief mention.

The most likely source of titanium is wall sputtering. Titanium emissions are independent of stabilizing techniques, as were carbon emissions (see above). This weighs against the streaming guns as a major source. There is no titanium in the neutral beam modules, so they cannot be the source. The machine walls remain as a possible source, although the particular mechanisms by which titanium is produced and penetrates the plasma are not known. The apparent broad spatial distribution of titanium (Appendix D) may be significant, because it suggests that the titanium originates in the magnetic fans, where penetration to central field lines is not difficult.

Nitrogen, in contrast to titanium, probably comes from the neutral

beams. The apparent broad spectral profile of $\mathrm{N} V 1242 \AA$ A emissions (Appendix C) and the broad spatial distribution of N III (Appendix D) are consistent with this hypothesis. A few experiments, like those reported in Section $V$ for oxygen, could disprove or substantiate it. 
A few conclusions may be drawn about the carbon, nitrogen, and titanium in 2XIIB. Some progress has been made toward understanding carbon, but the account is incomplete. Whatever the mechanisms related to the heating and transport of these impurities, their role in the central 2XIIB plasma was negligible because of their low concentrations. Their concentrations in the end fan regions are unknown, but could have been much larger. So Iong as a mirror reactor contains only high energy impurities, impurities should not be a problem (see Section VII). The sources and transport of carbon, titanium, and nitrogen are not yet well enough understood to be sure this will be the case. 


\section{G1. Introduction}

This Appendix provides detailed descriptions of the neon injection experiments, and a compilation of some of the data obtained. These experiments showed the rejection by the plasma of most of the impurities from certain sources, as was discussed in Section VI. The operation of 2 XIIB during these experiments is presented here. Some issues are also discussed which were not considered in Section VI. For instance, current theory of microinstabilities is compared to the results of the experiment in which neon plasma was used to stabilize the central plasma (see Section II). This experiment is discussed first; then the radial neon-puffing experiment is reviewed.

\section{G2. Injection of Neon-Streaming P1asma}

The streaming plasma required to maintain the high density plasma was supplied by neon gas arc guns during this experiment. These guns were also operated with deuterium on some shots, to provide a comparison. This section first describes the operation of $2 X I I B$. Then the neon data are presented and the penetration of the hot plasma by the neon, which was injected along field lines, is discussed. After this, the present understanding of the dxift-cyclotron loss-cone instability will be compared with the plasma characteristics observed when either neon or deuterium streaming plasma guns were used.

A schematic of 2XIIB operation during this experiment is shown in Fig. G1. The neutral beams were fired first, then a short pulse of the deuterium plasma guns was used to initiate the plasma buildup. These washer-stack-type guns were operated with a set of high voltage capacitor 
Timing of shot:

1. Magnetic field on, $(\sim 0 \mathrm{~ms})$

2. Neutral beams fire $(\sim 1.5 \mathrm{~ms})$

3. Neon plasma guns fire for $10 \mathrm{~ms}(\sim 2.5 \mathrm{~ms})$

$\mathrm{D}_{2}$ plasma guns fire for $1 \mathrm{~ms}(\sim 2.5 \mathrm{~ms})$

4. Neutral beams turn off $(\sim 9 \mathrm{~ms})$
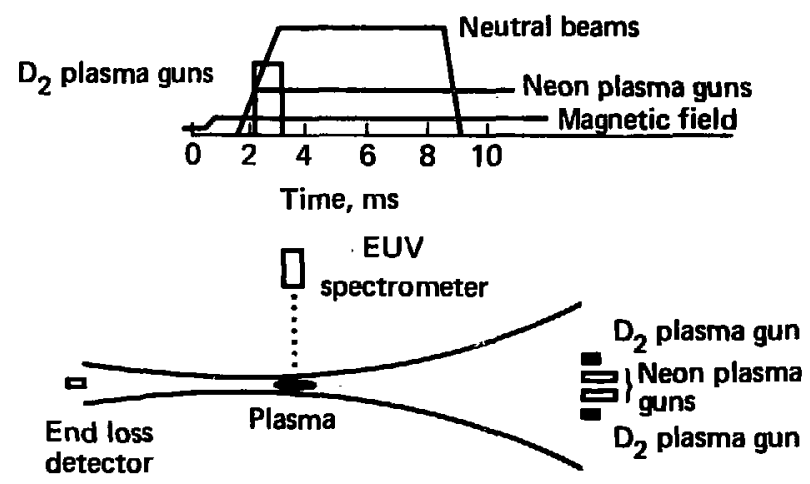

Figure G1. Schematic of the neon plasma injection experiment.

banks, which can be crowbarred effectively. The neon gas arc plasma guns were fired when the deuterium guns were, and were kept on throughout the shot to sustain the plasma.

The time development of some key plasma parameters is shown in Fig. G2. The electron line density, measured by microwave interferometry [ 57], increased gradually to a steady state that was maintained until the neutral beams were turned off. A 15-channel neutral analyzer measured the flux of high-energy neutrals produced by charge-exchange within the plasma [ 34$]$. The 25-keV charge-exchange-neutral flux was an indication of the fluctuation level, because beam-injected ions (with energies below $20 \mathrm{keV}$ ) must be heated by the rf fluctuations to reach this energy in measurable quantities. The plasma diamagnetism, measured by a large diamagnetic loop, indicated the amount of energy stored in the plasma. Neutral-beam attenuation measurements [58] showed a broad, Gaussian line density profile. Table Gl lists 

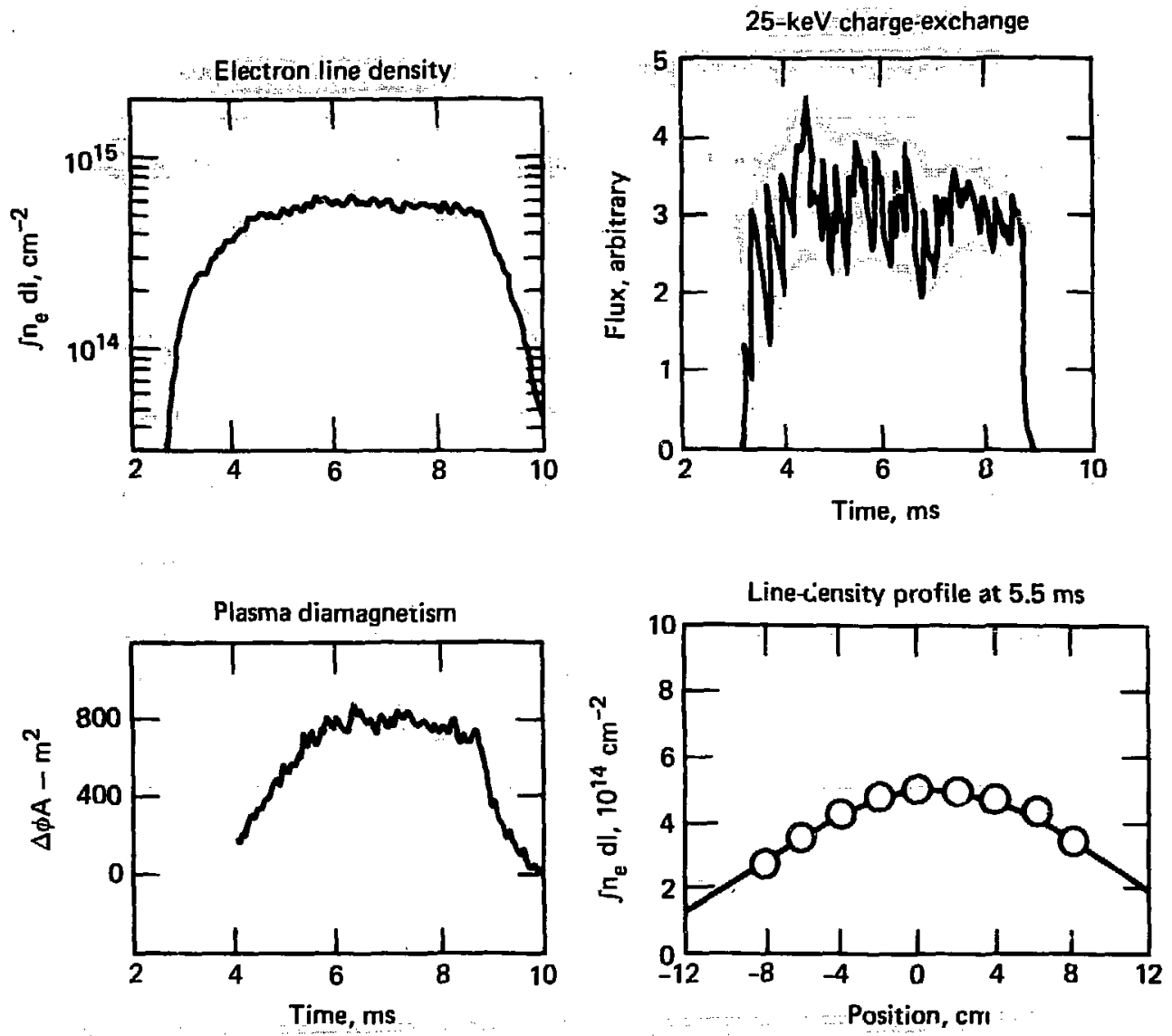

Figure G2. Plasma characteristics during neon plasma injection. 
TABLE Gl. PLASMA PARAMETERS DURING PLASMA INJECTION

\begin{tabular}{|c|c|c|c|}
\hline Parameter & Symbol & Neon-Stabilized & $\begin{array}{l}\text { Deuterium- } \\
\text { Stabilized }\end{array}$ \\
\hline Line Density & $\int n_{e} d 1$ & $5.5 \times 10^{14} \mathrm{~cm}^{-2}$ & $5.0 \times 10^{14} \mathrm{~cm}^{-3}$ \\
\hline Plasma Radius & $R_{p}$ & $12.5 \mathrm{~cm}$ & $11 \mathrm{~cm}$ \\
\hline Peak Density & $\hat{n}_{e}$ & $2.6 \times 10^{13} \mathrm{~cm}^{-3}$ & $2.6 \times 10^{13} \mathrm{~cm}^{-3}$ \\
\hline Electron Temperature & $\mathrm{T}_{\mathrm{e}}$ & $50 \mathrm{eV}$ & $50 \mathrm{eV}$ \\
\hline $\begin{array}{l}\text { West End Loss } \\
\text { At Midplane }\end{array}$ & j & $40 \mathrm{~mA} / \mathrm{cm}^{2}$ & $100 \mathrm{~mA}-\mathrm{cm}^{-2}$ \\
\hline Neutral Beam Current & $I_{b}$ & $390 \mathrm{~A}$ & $390 \mathrm{~A}$ \\
\hline Plasma Diamagnetism & $\Delta \phi$ & $720 \mathrm{~A}-\mathrm{m}^{2}$ & $720 \mathrm{~A}-\mathrm{m}^{2}$ \\
\hline Mean Ion Energy & $\mathrm{W}$ & $12 \mathrm{keV}$ & $10 \mathrm{keV}$ \\
\hline Neon Density & $\mathbf{N}$ & $6.8 \times 10^{10} \mathrm{~cm}^{-3}$ & \\
\hline
\end{tabular}


the characteristics of these plasmas during steady state, for both neon- and deuterium-stabilized plasmas. The Thomson scattering diagnostic measured electron temperature and peak electron density [59]. The same value of peak electron density was also obtained from the beam-attenuation and microwave diagnostics. The end loss current, which is significant in the discussion of stability below, was measured with a gridded analyzer [60]. The known grid transmissions and magnetic field variations were used to determine the westward current at the midplane from the measured current in the analyzer. The time development of emissions observed when the neon plasma guns were used is shown in Fig. G3. Ne II $460 \AA$ emissions show the behavior described in Section $V$; the apparently negative signal during steady state is seen because the electron density was slightly higher during the shot

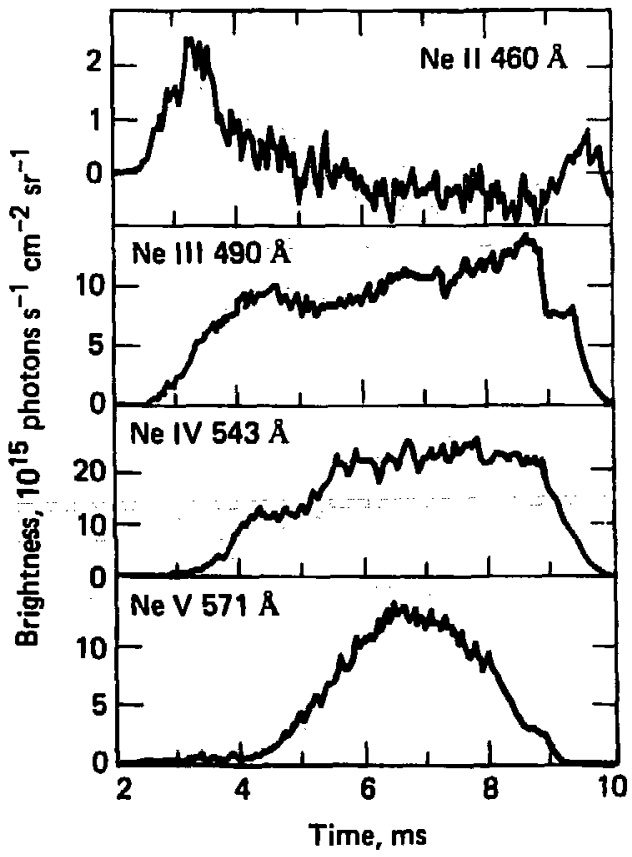

Figure G3. The time development of emissions from Ne II through Ne $V$ during plasma injection shows the successive appearance of emissions from more highly ionized states. 
used for background subtraction. The time, of rise of the neon emissions increased with successively higher ionization state, as was expected. Ne VI $402 \AA$ emissions were small and were not resolved from background, Note that the observed oxygen brightnesses reported in Appendix c were much larger than the neon brightnesses produced when the streaming plasma was pure neon. Any oxygen in the streaming plasma evidently contributed little to the total observed oxygen brightness.

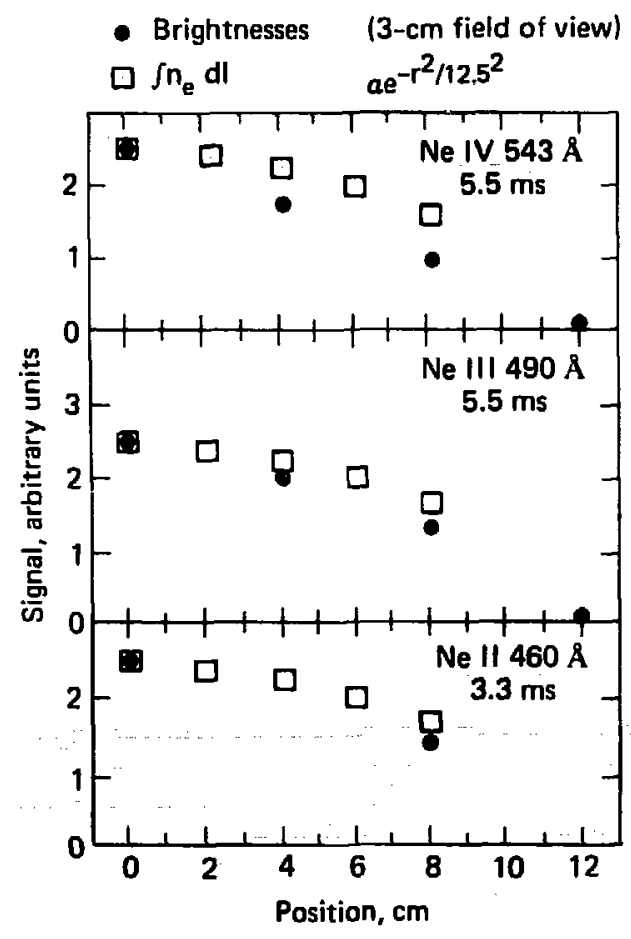

Figure G4. Spatial scans during plasma injection show the broad distribution of the neon. 
We performed some spatial scans of neon emissions; Fig. G4 shows the results. The neon spatial profiles are broad and are not hollow (although this data data does not rule out some shell structure). This is not surprising for axially injected impurities. This spatial data is insuffciently detailed to draw any precise conclusions about the spatial profiles of the neon.

Plasmas were created using only the neon plasma guns and magnetic fields but not the neutral beams, in order to estimate how much of the neon plasma penetrated the high-density beam-fueled plasma. The plasma gun produced an elliptical plasma (as a result of magnetic field mapping); its parameters were $T_{e} \sim 5 \mathrm{eV}, n_{e} \sim 1.6 \times 10^{13} \mathrm{~cm}^{-3}, E_{i} \leq 250 \mathrm{eV}$. The line density along the minor axis of the ellipse was $\int n_{e} d l=2.5 \times 10^{13} \mathrm{~cm}^{-2}$. The size of the ellipse along the major axis, w, is $13 \mathrm{~cm}$. Because of the low electron temperature, this plasma is assumed to be entirely Ne II. T: number of neon ions per unit length, $\rho$ gun, is approximately

$$
\rho_{\text {gun }}=\int \mathrm{n}_{\mathrm{e}} \mathrm{dl} \times \mathrm{w}=3.3 \times 10^{14} \mathrm{~cm}^{-1} \text {. }
$$

The number of neon ions per unit length penetrating the beam-fueled plasma is approximately

$$
\rho_{\text {pen }}=\mathrm{N}_{\pi} \mathrm{R}_{\mathrm{Ne}}^{2}=1.4 \times 10^{13} \mathrm{~cm}^{-1},
$$

in which $\mathrm{R}_{\mathrm{Ne}}$ is the neon radius $(=8 \mathrm{~cm})$ determined by the spatial scans. The neon density, $N$, is determined by the constant density model described in Appendix C, using rate coefficients calculated by Dr. J. Davis [ 39]. Table G2 shows the neon density results. The ratio of Eq. (G2) to Eq. (G1) gives the fractional neon penetration, which was about $4 \%$. 
TABLE G2. NEON DENSITIES DURING PLASMA INJECTION

(evaluated at $5.5 \mathrm{~ms}$ )

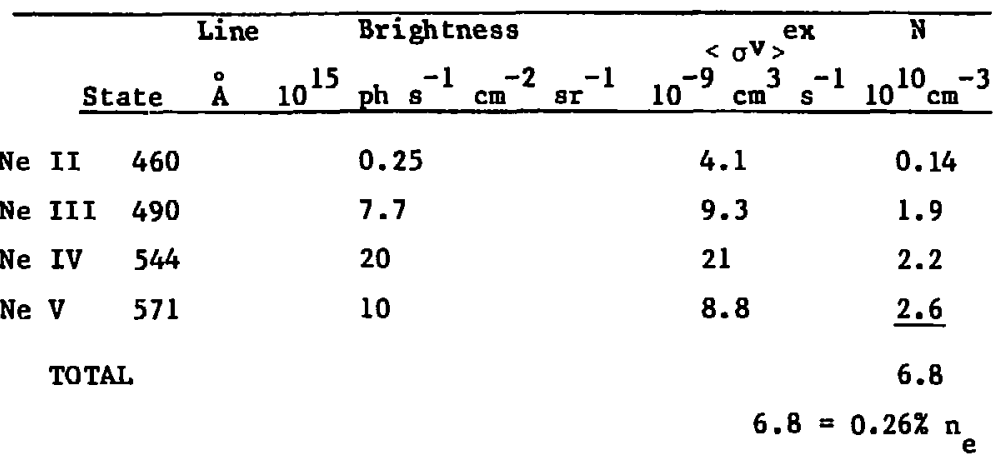

Before proceeding to considerations of stability, the above results can be summarized. The hot, high-density plasma prevented the penetration of all but a small fraction of the injected neon plasma to the midplane. The evenly distributed neon emissions which resulted were much less bright than the observed oxygen emissions, and the Ne II emissions showed the expected attenuation of the once-ionized neon flux as plasma density increased. The neon concentration was only $0.3 \%$ in the hot plasma, which suggests that the streaming plasma could not be the major source of the observed carbon and titanium. When the streaming plasma was mostly deuterium, these elements had concentrations near $0.3 \%$ at the midplane. By comparison with the neon result, these concentrations appear too high to have come from a streaming plasma source.

Some considerations of plasma stability follow. The fact that the 2XIIB plasma could be maintained using a neon streaming plasma is itself significant. This means that the presence of externally supplied low-energy deuterium ions is not necessary to maintain the plasma. If the only role of the streaming plasma is to supply particles that fill the ambipolar hole in velocity space, then this is a surprising result, because current theory 
requires that these particles be resonant with the deuterium-ion-cyclotronfrequency fluctuations [4]. Neon particles are not resonant, and should have little stabilizing effect. The plasma cannot be maintained with no stream, [ 2], but can be maintained with a neon stream. It may be that the only role of the neon stream is to cool the electrons, lowering the required density of low-energy deuterons to that which the plasma can supply by means of an increased fluctuation level (see below). Even if this is true, it will now be shown that the low-energy deuteron density during these experiments was $f$ ar below that required by current theory.

The end-loss current measurements can be used to estimate the density of low-energy deuterons at the midplane of 2XIIB. This diagnostic measured the flux and energies of particles moving in one direction (see below). By assuming there was an equal flux in the opposite direction, and neglecting the contribution of the neon ions to the measured current, an upper limit may be placed on the low energy deuteron density $\left(n_{W}\right)$. Figure G5 shows end-loss analyzer data obtained during this experiment by varying the voltage of a repeller grid, to reject ions with energies below the grid voltage. Because the current did not drop until the repeller voltage exceeded $150 \mathrm{~V}$, this data is consistent with a plasma potential near $3 \mathrm{~T}_{\mathrm{e}}$. The median detected energy was $800 \mathrm{eV}$, which corresponds to $650 \mathrm{eV}$ at the midplane, where the potential is maximum. Assuming that a total deuteron current of $80 \mathrm{mAcm}^{-2}$ flowed out both ends of the machine with a mean energy of $650 \mathrm{eV}$, $\mathrm{n}_{W}$ at the midplane is $2 \times 10^{10} \mathrm{~cm}^{-3}\left(=0.08 \% \mathrm{n}_{e}\right)$. This estimate should be about a factor of 2 high, because the neon current should be 10 to $50 \mathrm{mAcm}^{-2}$ depending on the neon energy. (There are other uncertainties as well.) The low-energy deuterons are provided by the trapped neutral beam particles. The neutral beam trapping rate is equivalent to a $\mathrm{flux}$ of $120 \mathrm{mAcm}^{-2}$, but some or all of these particles may leave the machine radially. 


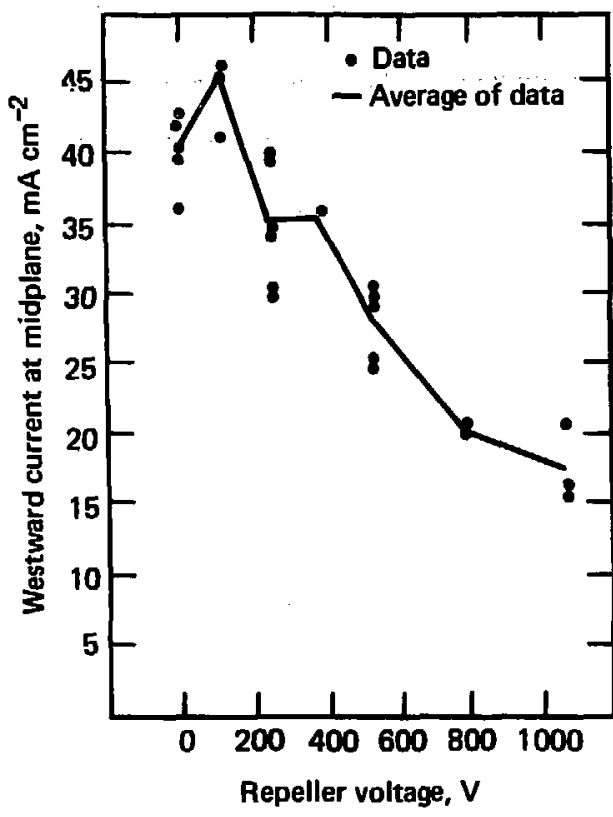

Figure G5. During the neon plasma injection experiment, the ion end loss current and energy distribution were determined using a gridded analyzer. The westward current is normalized to the midplane magnetic field. The increase in measured current from zero to 140 volts repeller voltage is unphyical, and is indicative of the scatter in the data.

The theoretical warm density requirement may now be compared with the value obtained above. The required warm density is:

$$
\frac{n_{w}}{n_{e}}=0.3 \frac{e \phi}{E_{i}}=0.4 \%
$$

(The factor 0.3 is below the lower limit of present theory [5]). Thus, the low-energy deuteron density in these plasmas was a factor of five below the theoretical requirement. This difference is large compared to the uncertainties. In addition, most of the observed low-energy deuterons were not in the ambipolar hole (they had too much energy). If this is possible at higher densities and temperatures, future mirror machines may be able to avoid the large power drain associated with present stabilizing techniques, by using better controlled sources of low energy deuterons. 
The neon-stabilized plasmas may be compared with the plasmas created when deuterium was used in the gas arc guns. The plasmas stabilized by deuterium had a lower fluctuation level than those stabilized by neon, although most of the plasma parameters were nearly identical. The lower fluctuation level of the deuterium-stabilized plasmas is shown in Fig. G6. As was discussed above, the $25 \mathrm{keV}$ charge-exchange flux indicates the instability-induced ion heating. The deuterium-stabilized plasma produced less of this flux. The rf fluctuations also scatter the $2 \mathrm{~mm}$ microwaves used for line density measurements; the deuterium-stabilized plasma produced less microwave scattering, indicating less intense fluctuations. In addition, as was shown in Table Gl, the deuterium-stabilized plasmas had lower mean ion energies and smaller radii than those stabilized using neon. This may indicate less instability-induced diffusion, because such diffusion tends to increase the mean ion energy and plasma size. Despite these differences, the neon-stabilized plasma adpated itself to the relative absence of low-energy deuterons without substantial alteration. The energy and particle confinement times were essentially unchanged.

(The density of low-energy deuterons is also below the theoretical requirement in the deuterium-stabilized case. The westward current is $100 \mathrm{~mA} \mathrm{~cm}{ }^{2}, 2.5$ times above the previous value. Thus, $n_{w} / n_{e}$ is one-half the theoretical requirement, if the same analysis applies. This is again an over-estimate of $\mathrm{n}_{\mathrm{w}} / \mathrm{n}_{\mathrm{e}}$, because some of the current is supplied by the gas arc gun and only flows in one direction.)

\section{G3. Radial Puffing}

The results of this experiment are considered at length in section VI. The machine operation and the data obtained are discussed here. The pulsed 


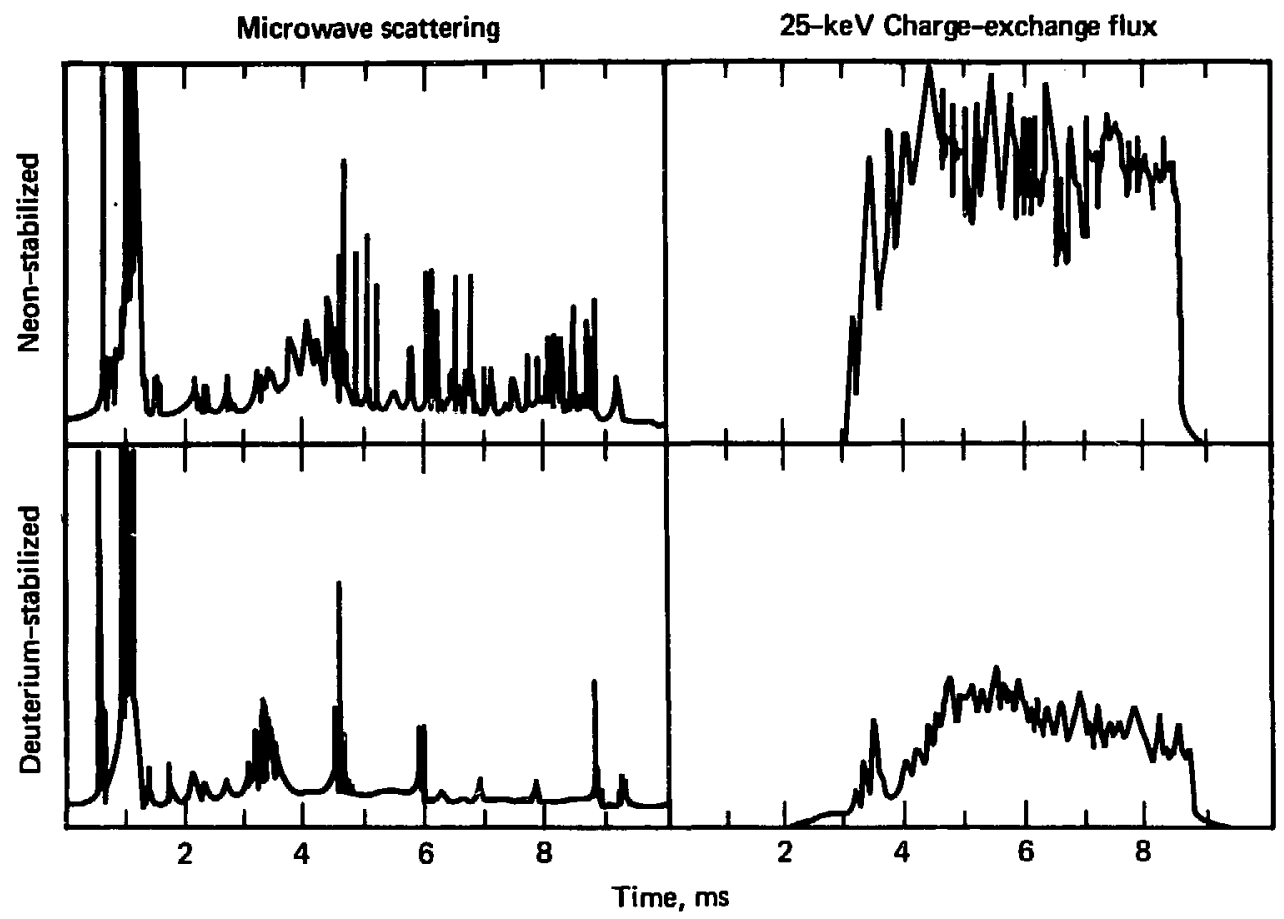

Figure G6. When neon was used, instead of deuterium, to stabilize the plasma, more intense fluctuations were observed.

valve, the plasma, and the EUV monochromator were oriented as shown in

Fig. G7. It is clear that if the neon is deposited on the boundary and soon leaves the machine, the neon spatial profiles should be asymetric. Figure G8 shows spatial scans of 2 XIIB, and asymmetric profiles were observed. The details of the spatial profiles will depend on azimuthal drifts, radial transport, and axial loss; this spatial data is insufficiently precise to address these issues.

The amount of gas injected into the plasma was varied by changing the pressure in the gas supply line. This changed both the neon brightness and the effects of the neon gas. When the supply pressure was 50 torr, the neon current trapped by the plasma $(18.6 \mathrm{~cm}$ by $40 \mathrm{~cm}$ ) was about 10 equivalent A. 
$6 \mathrm{~cm} \mathrm{~S}, \mid-13^{\circ} / \begin{gathered}\text { EUV line of } \\ \text { sight } \\ \text { Central } \\ \text { chord }\end{gathered}$

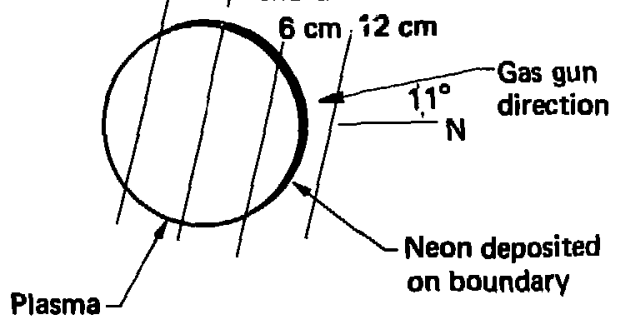

Looking west

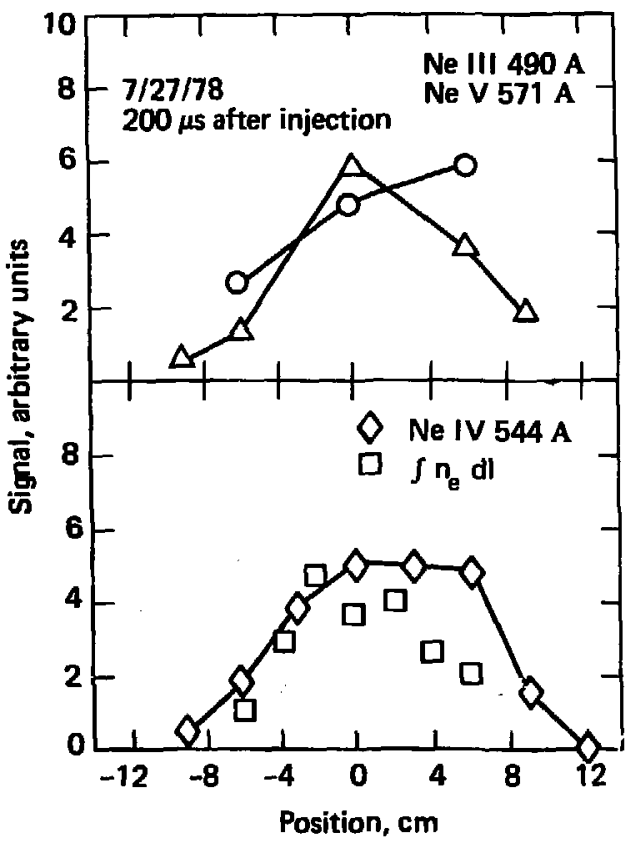

Figure G7. Schematic of the neon gas puffing experiment.
Figure G8. Spatial data obtained during neon gas puffing showed asymetric brightness profiles. 
The Ne IV $544 \AA$ time development, obtained using three different gas pressures is shown in Fig. G9. The signal at $3.5 \mathrm{~ms}$ is background, and the increase after this time was measured. Figures G10 and G11 show similar time histories for Ne V $571 \AA$ and Ne III $490 \AA$.

Ne IV $544 \AA$
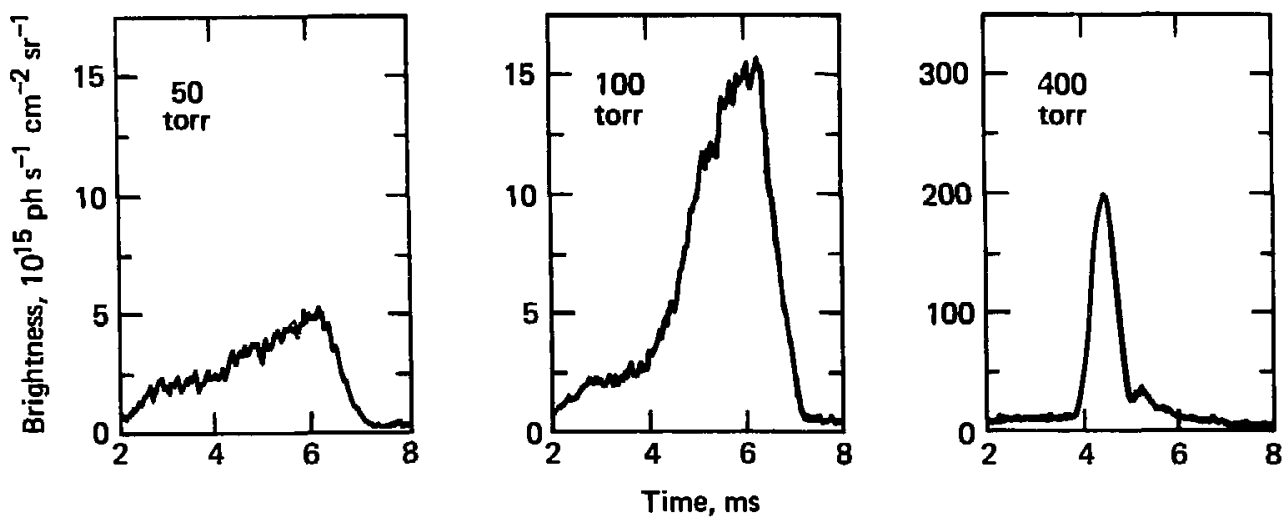

Figure 69. The Ne IV $544 \AA$ time development was observed as the gas pressure in the gas valve supply line was increased. The signal prior to $3.5 \mathrm{~ms}$ is background.

The effects of the neon gas on the plasma are shown in Figs. G12 and G13. The plasma diamagnetism is an indicator of the number of stored ions, and decreases more rapidly as the neon gas $f 1: a x$ is increased. The electron line density, measured by beam attenuation, is affected very little except at the largest neon fluxes, which destroy the plasma. The energy distribution of the deuteriun ions, measured by the charge-exchange neutral analyzer, is not affected until major effects are observed in the electron density. The east end losses increase slightly when the neon gas strikes the plasma, and are eliminated when the plasma is destroyed. 

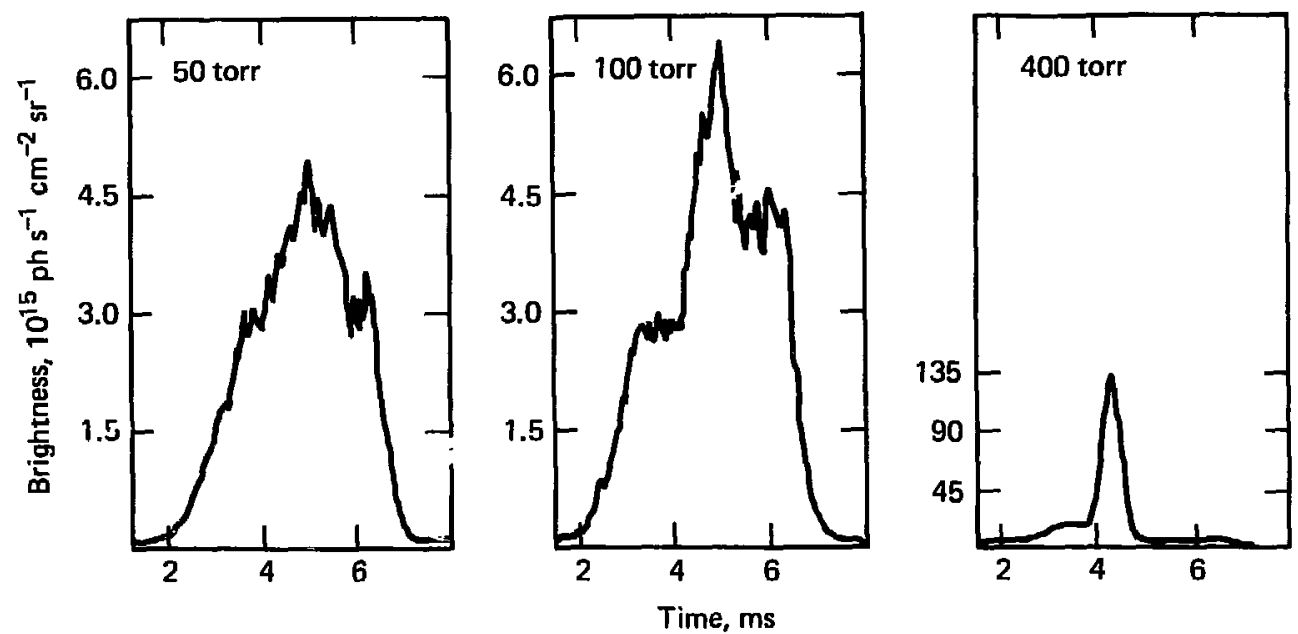

Figure G10. The Ne V $571 \AA$ emissions increased as the gas-valvesupply pressure was raised, which increased the neon flux striking the plasma.
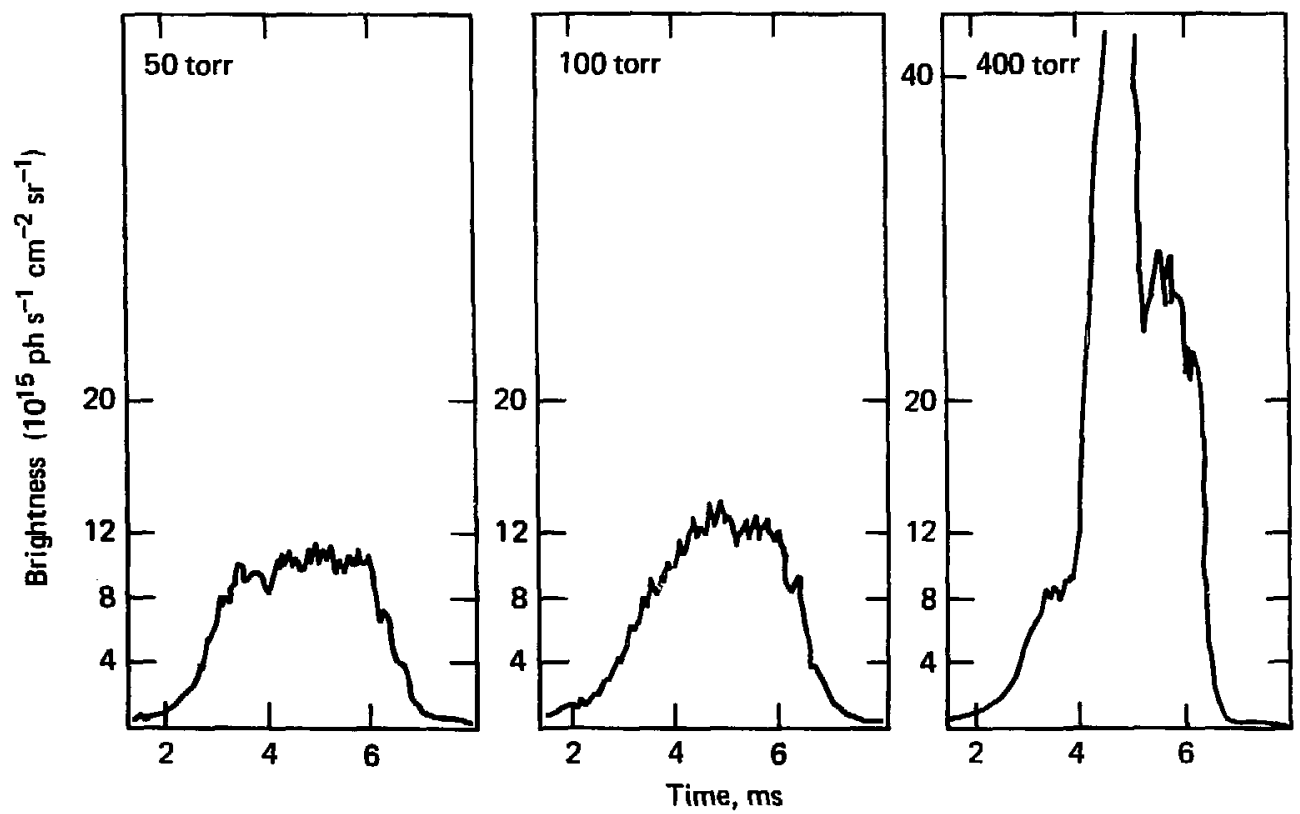

Figure G11. The Ne III $490 \AA$ brightness increased when the neon gas flux was raised by increasing the pressure in the gas-valve-supply line. 
Plasma

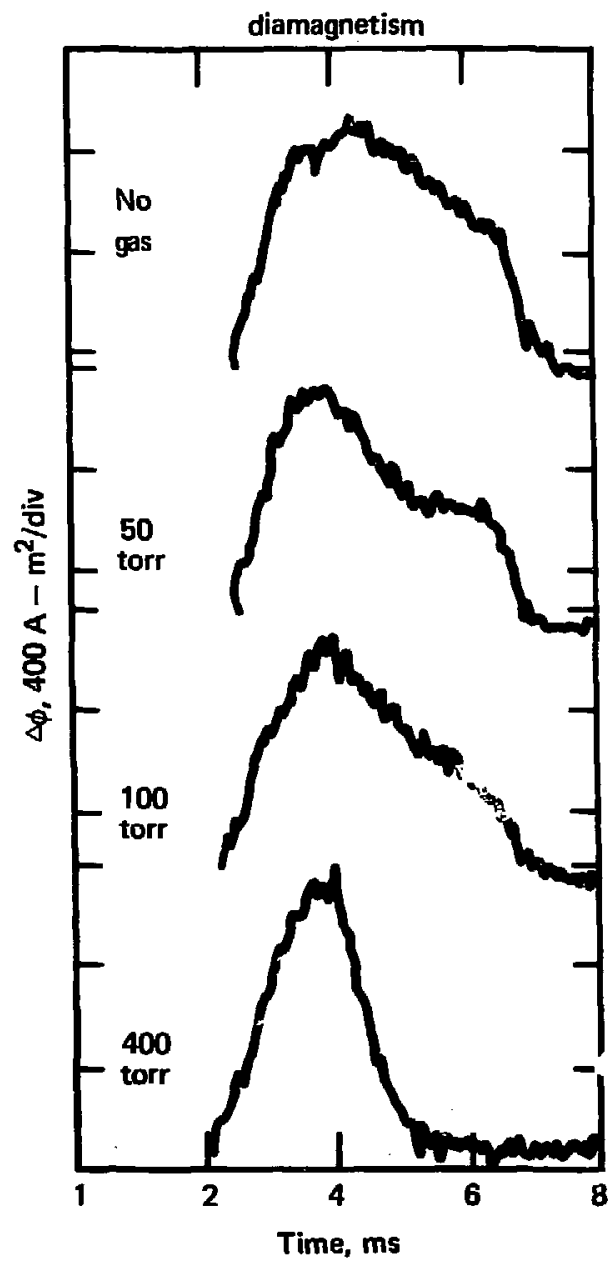

\section{Electron}

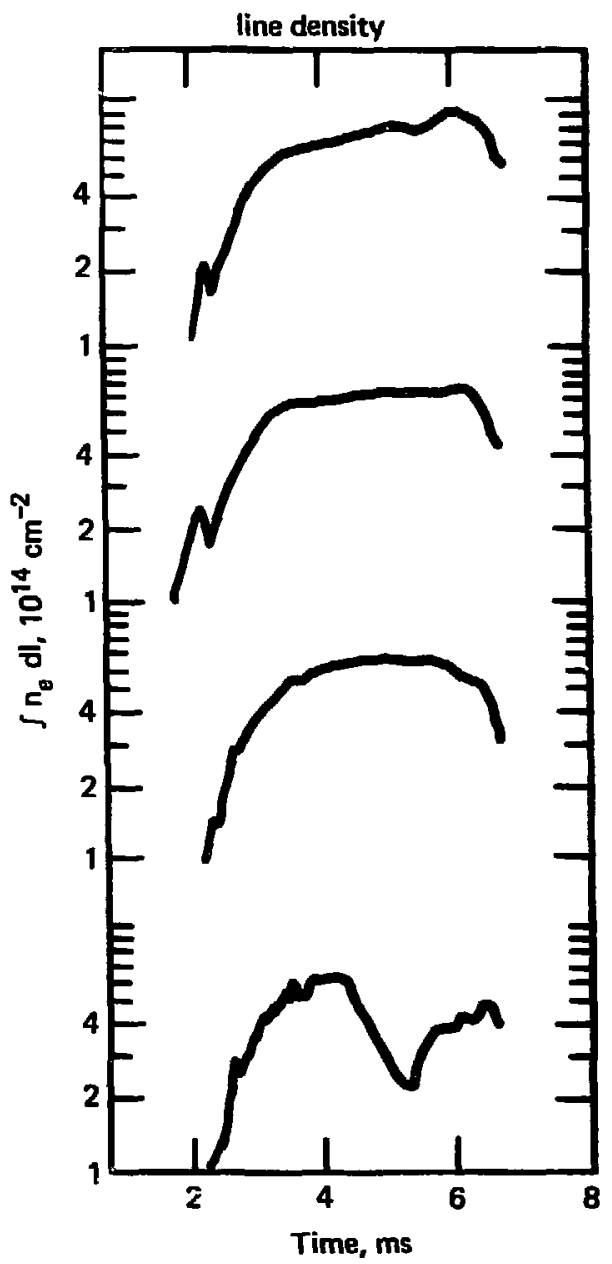

Figure G12. The neon flux striking the plasma was increased by raising the gas valve supply pressure ( 0 to 400 Torr were used). Plasma diamagnetism and electron line density are shown. 
Energy distributions (at $5 \mathrm{~ms}$ )

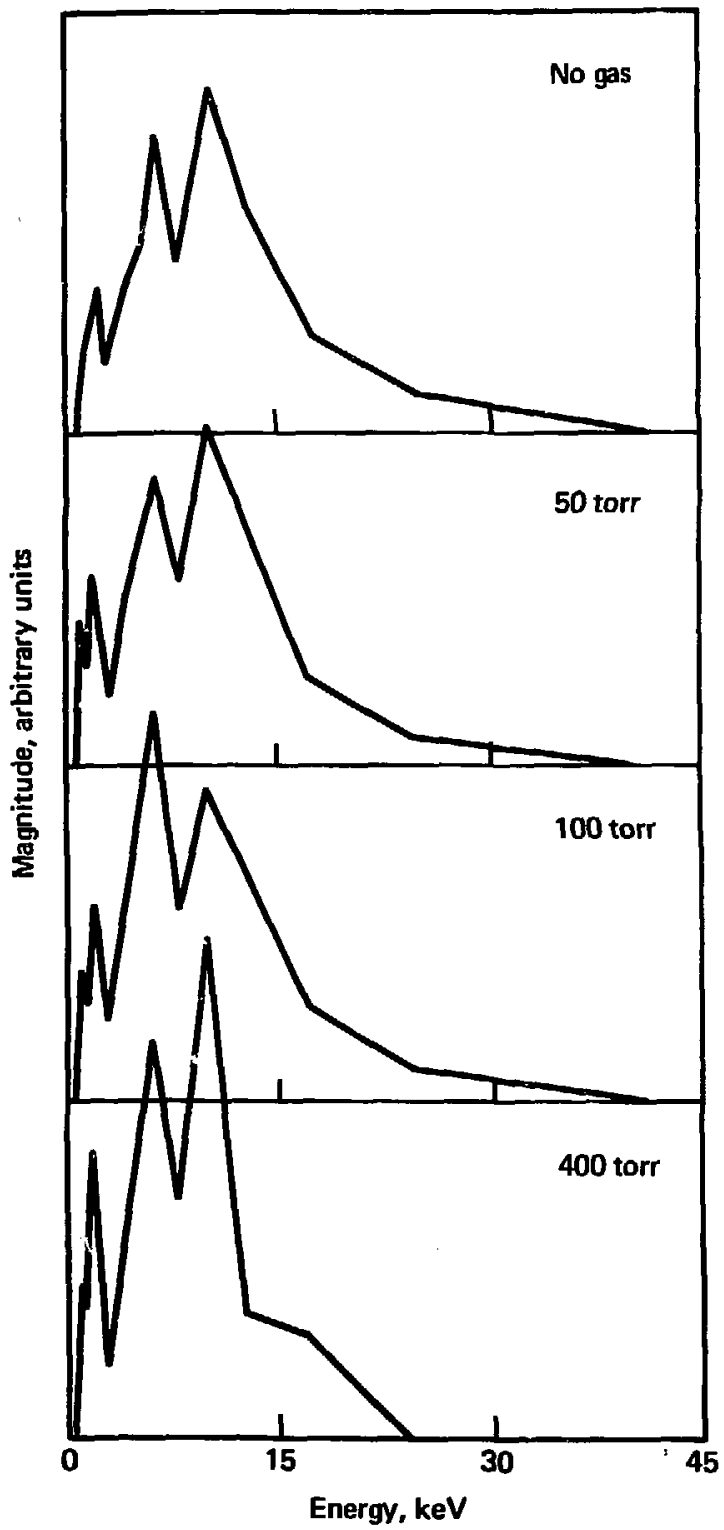

East-end losses

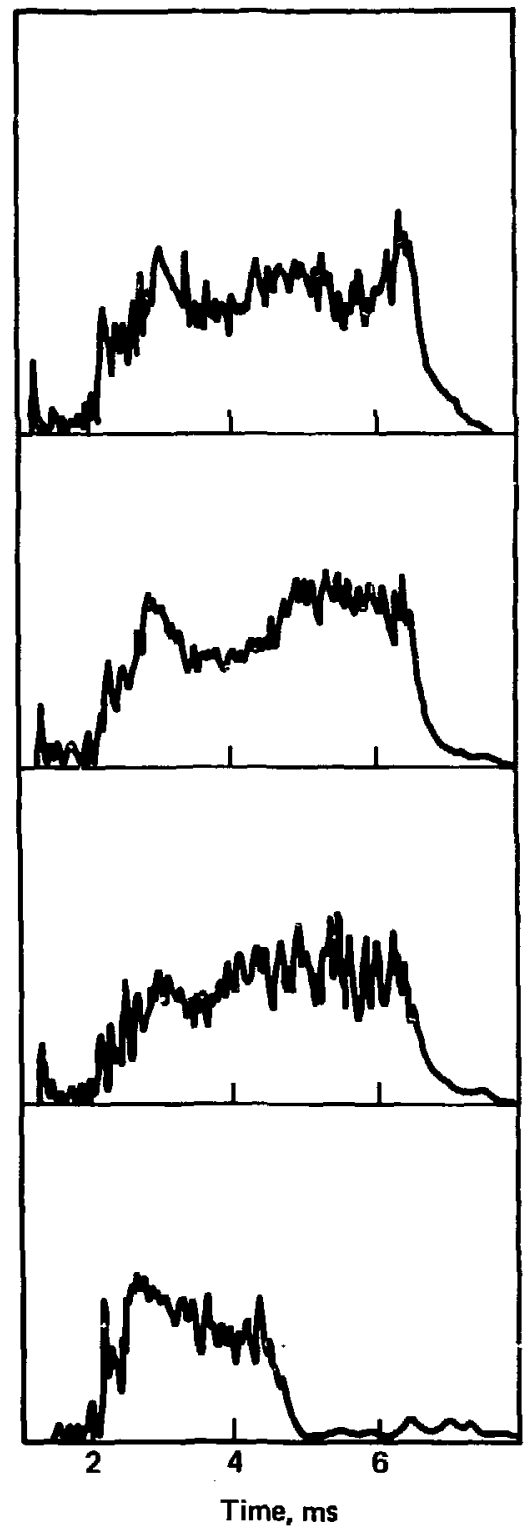

Figure 613. The energy distributions and end. loss currents were affected little until the plasma was destroyed. 
A gas supply pressure of 400 torr, leading to about $100 \mathrm{~A}$ of trapped neon current, was sufficient to destroy the plasma (and still produced neon brightnesses less than those of oxygen). Thi: data was not used in the analysis of Section VI. By contrast, 50- or 100-torr supply pressures resulted in slow changes in the plasma parameters. The data from these shots was analyzed at $5 \mathrm{~ms}$, when a steady state had nearly been reached in the 50 torr case. Although the neon did affect the plasma, the plasma was able to maintain itself for long times compared to the neon confinement time. The analysis of Section VI is not invalidated by such long-term effects. 


\section{H1. Introduction}

Some of the EUV data apply to the boundary region of the plasma; such data are discussed in this Appendix. As noted in Appendix $C$, the plasma boundary in 2 XIIB was not we11-diagnosed. In certain respects, the EUV data indicate the nature of this region. The neon-gas-puffing results suggest that the electron temperature was not much lower than the central value at a radius of about $9 \mathrm{~cm}$. The impurity spatial data indicated that either electron temperature or density decreased significantly just beyond a radius of $9 \mathrm{~cm}$. Lyman alpha measurements suggest that the plasma was struck by significant low-energy neutral fluxes ( 1 to $3 \times 10^{17} \mathrm{~cm}^{-2} \mathrm{~s}^{-1}$, depending on neutral-beam current). These points are discussed below; they might be explained by the existence of a boundary where plasma ions are lost by charge-exchange with low-energy deuterium. This boundary would exist where the flux of plasma ions diffusing outward balances the inward flux of low-energy neutral atoms, and the electron temperature and density would both decline; the rate of sross field transport of energy and particles would determine the sharpness of the boundary.

The neon data discussed in section VI suggest that the $T_{e}$ spatial profile is broad. The fact that Ne V $571 \AA$ emissions are seen as soon as Ne III $490 \AA^{\circ}$ emissions indicates that $T_{e}$ is substantial $(\sim 50 \mathrm{eV})$ in the emitting region, which is near a radius of $9 \mathrm{~cm}$ (see Appendix G). Thomson scattering measurements have shown that $T_{e}$ declines slowly out to a $6 \mathrm{~cm}$ radius [5]; the EUV data extends this result to about $9 \mathrm{~cm}$. (Consistent with the neon data, $T_{e}$ could begin to decline rapidly anywhere outside of an $8 \mathrm{~cm}$ radius.) 
It appears that the electron density profile is Guassian and the electron temperature declines slowly. Yet somewhere the electron temperature must drop, and the electron line density was not measured beyond $8 \mathrm{~cm}$. The impurity spatial scans place some limits on these quantities. In particular, consider the 0 II $539 \AA$ spatial scan reported in Section V (see Fig. 9). Given the high energy of the oxygen, the 0 II had a large orbit $\left(r_{L}=11 \mathrm{~cm}\right)$. As a result, the 0 II density must decline slowly as radius increases. The brightness of $539 \AA$ emissions at $r=12 \mathrm{~cm}$ is at most $3 \times 10^{14} \mathrm{ph} \mathrm{s}^{-1} \mathrm{~cm}^{-2} \mathrm{sr}^{-1}$. Applying Eq. III-9,

$$
3 \times 10^{14}>\frac{1}{4^{\pi}} N \int_{\mathrm{e}} \mathrm{dl}<\sigma_{\mathrm{v}}>\operatorname{ex} .
$$

On the basis of the data discussed in Section V (Fig. 9), one can assume $\mathrm{N}=10^{10} \mathrm{~cm}^{-3}$, then

$$
\left.\int n_{e} d l<\sigma v\right\rangle^{e x}<3.8 \times 10^{5}
$$

If $\int n_{e} d l$ remains Gaussian beyond $8 \mathrm{~cm}$, where it is not measured, $T_{e}$ must be below $20 \mathrm{eV}$ at a radius of $12 \mathrm{~cm}$ to satisfy $\mathrm{Eq}$. (H1). If $\mathrm{T}_{\mathrm{e}}$ remains high, then the line density must be a factor of 2 below the Gaussian prediction. We observe that either the temperature or the density (or some combination) declines sharply at large radii $\left(r>R_{p}\right)$. Although these cases are not distinguis:.jble, either is sufficient to establish a "boundary" at $r \simeq R_{P}$, because radial gas $f l u x e s$ can then penetrate that far into the plasma. 
Other impurity spatial scans support the conclusions drawn from the 0 II spatial scan. The brightness at $r=12 \mathrm{~cm}$ is $5 \%$ or $1 \mathrm{ess}$ of the peak brightness, while(for $R_{p}=10 \mathrm{~cm}$ ) the line density should be $20 \%$ of its peak value. If the line density is this high, then $\mathrm{T}_{\mathrm{e}}$ must be low $(20 \mathrm{eV})$, either to avoid emitting more strongly or to avoid creating the observed ions. If $T_{e}$ is high, the line density must be lower than that predicted by the central Gausian profile. Either case is sufficient to establish a boundary.

H3. Implications of the Lyman Alpha Data

Under proper conditions, the Lyman alpha brightness is directly proportional to the cold gas flux. This provides some indication of the amount of 10 energy deuterium striking the plasma. This situation, and the data obtained from 2 XIIB, are discussed below.

The relation of the Lyman alpha brightness to the cold gas flux is evaluated first. When the neutral beams are on, the presence of high energy neutral atoms makes the Lyman alphe brightness and spectrum quite complicated [50]. However, once the beams have been turned off (which takes $\sim 100 \mu s)$, the density of high-energy neutrals becomes negligible very soon $(25 \mu s)$. In this case, by a derivation like that of section VI, the flux, $\Gamma$, is found to be

$$
\Gamma=\frac{\left.2 \pi B\left(<\sigma v>^{i o n}+f_{h}<\sigma v\right\rangle^{c x}\right)}{<\sigma v\rangle^{e x}}
$$

In this equation, $f_{h}$ is the high-energy fraction of deuterons in the region where the cold gas is stopped. (Only the high-energy deuterons charge-exchange strongly). For the moderate fluxes present in $2 \times I I B, f_{h}{ }^{n} 1$. 
The flux of low-energy deuterium was measured by this technique under two different circumstances during this experiment. Figure Hl shows a typical Lyman alpha time development, taken with slits which were wide enough to include the entire line. When the neutral beams turn off, the Lyman alpha brightness abruptly declines and soon reaches about $10^{16} \mathrm{ph} \mathrm{s}^{-1} \mathrm{~cm}^{-2} \mathrm{sr}^{-1}$. This signal could result from room temperature fluxes $\left(v \sim 10^{5} \mathrm{~cm} \mathrm{o}^{-1}\right)$ which had not yet dissipated, or from wall reflux $\left(v 10^{6} \sim \mathrm{cm} \mathrm{sec}^{-1}\right)$ produced during the plasma decay. The fluxes corresponding to the Lyman alpha brightnesses observed $1 \mathrm{~ms}$ after beam turn-off are shown in Table Hl. The beam current and approximate emission radius are also listed.

These fluxes, multiplied by the plasma area, give an indication of the low-energy deuterium current striking the plasma. For the data shown in Fig. H1 (assuming a $30 \mathrm{~cm}$ plasma length), this current is about 70 equivalent A. A comparable current of low-energy deuterium may strike the plasma during the high density plateau, because similar sources are present. In that event, any theory of the plasma boundary must explain how that deuterium interacts with the plasma. 


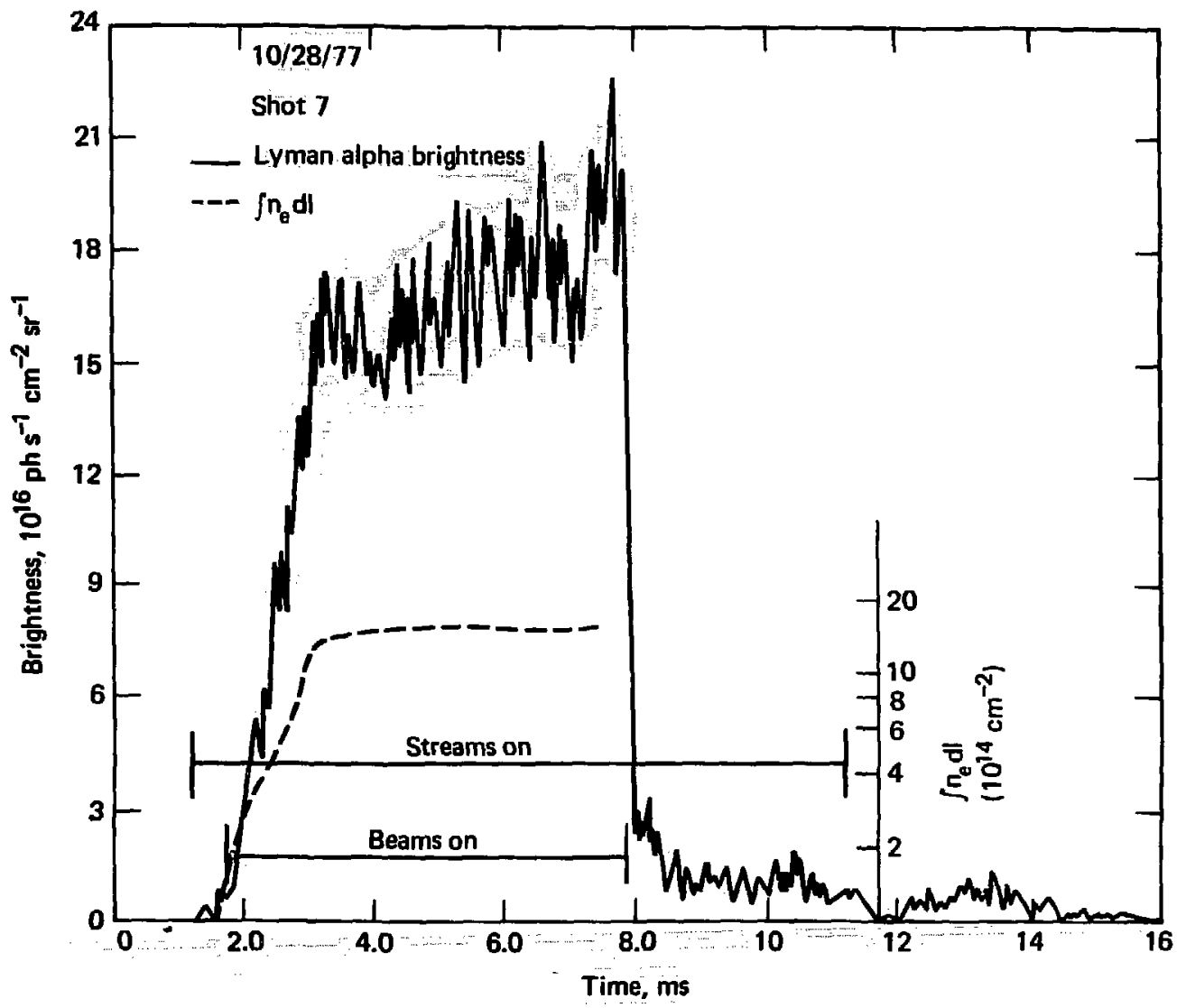

Figure Hl. The Lyman alpha time development, electron line density, and beam and stream durations are shown. Wide slits were used to measure the entire line. 
TABLE HL. LOW-ENERGY DEUTERIUM FLUXES

\begin{tabular}{cccccc}
\hline & & $I_{b}$ & $R$ & B & $\Gamma$ \\
Date & Shot & A & $\mathrm{cm}$ & $\mathrm{ph} \mathrm{s}^{-1} \mathrm{~cm}^{-2} \mathrm{sr}^{-1}$ & $\mathrm{~cm}^{-2} \mathrm{~s}^{-1}$ \\
\hline $10 / 28$ & 7 & 490 & 8 & $1 \times 10^{16}$ & $3 \times 10^{17}$ \\
$4 / 13$ & 28 & 330 & 11 & $4 \times 10^{15}$ & $1.2 \times 10^{17}$ \\
\hline
\end{tabular}




\section{BIBLIOGRAPHY}

1. CoEnsGen, F. H., et a1., Nuc. Fus. Supplement, Part I, 125 (1962).

2. COENSGEN, F. H., CLAUSER, J. F., CORRELL, D. L., CUMMINS, W. F., GORmezano, C., et al., Plasma Physics and Controlled Nuclear Fusion Research (Proc. 6th Int. Conf. Berchtesgaden, Germany, 1976) 스, 135.

3. SIMONEN, T. C., COENSGEN, F. H., CUMMINS, W. F., MOLVIK, A. W., NEXSEN, W. E., Nuc. Fus. 15813 (1975).

4. BALDWIN, D. E., Rev. Mod. Phys. 49317 (1977).

5. CORRELL, D. L. et al., "Results of 2XIIB Plasma Radius Scaling Experiments," submitted to Nuc. Fus.

6. Clauser, J. F., Bull. Am. Phys. Soc. 23850 (1978).

7. TURNER, W. C., Journal de Physique, Colloque C6, Suppl. 12, Tome 38, C6-121 (1977).

8. COENSGeN, F. H., CUMMINS, W. F., LOGAN, B. G., MOLVIK, A. W., NEXSEN, W. E., SIMONEN, T. C., Phys. Rev. Lett. 351501 (1975).

9. STALlARD, B. W., COENSGen, F. H., CUMMINS, W. F., GORMEZANo, C., LOGAN, B. G., et E. $_{-}$, Lawrence Livermors Laboratory report UCRL-7B741 (1976).

10. SIMONEN, T. C., CLAUSER, J. F., COENSGEN, F. H., CORRELL, D. L., CunMins, W. F., et al., Plasma Physics and Controlled Nuclear Fusion Research (Proc. 7th Int. Conf., Innsbruck, Austria, 1978).

11. TURNer, H. C., ClAUSER, J. F., COENSGEN, F. H., CORRELL, D. L., CUMmIN, H. F., et al., Nuc. Fus. 191011 (1979).

12. CLAUSER, J. F., et al., "Results of 2XIIB Electron Temperature Scaling Experiments," paper in preparation.

13. GRUBB, D. P., et al., Bull. Am. Phys. Soc. 23775 (1978). 
14. MOOS, H. พ., CHEN, K. I., TERRY, J. Ғ., FASTIE, W. G., "Construction, Calibration and Application of a Compact Spectrophotometer for EUV (300-2500 A) Plasma Diagnostics," Applied Optics 181209 (1979).

15. RICHARDS, R. K., Rev. of Sci. Inst., 491210 (1978).

16. SALOMAN, E. B., EDERER, D. L., Applied Dptics 141029 (1975).

17. ISLER, R. C., CRUME, E. C., Phys. Rev. Lett. 411296 (1978).

18. MAGEE, N. H., MANN, J. B., MERTS, A. L., ROBB, W. D., LOS Alamos Scientific Lab. Report LA-6691-MS (1977).

19. MATTIOLI, M., EURATOM Report EUR-CEA-FC-761 (1975).

20. DAVIS, J., JQSRT 14, 549 (1974).

21. DAVIS, J., KEPPLE, P. D., BLAHA, M., JQSRT 151145 (1975).

22. DAVIS, J., Naval Research Lab. Report No. 33.

23. GABRIEL, A. H., JORDAN, C., Ch. 4., Vo1. 2, CASE STUDIES IN ATOMIC COLLISION PHYSI'S, Eds. E. McDaniel and M. McDowe11, North Holland, Amsterdam, 209 (1971).

24. LOTZ, W., Astroph. J., Supp1. 128, 14, 207 (1967).

25. Jones, L. A., Proc.of the 2nd Top. Conf. on Atomic Processes in High Temperature Plasmas, Paper G-2, p. 49 (1979).

26. Crandall, D. H., PHANeUf, R. A., MEYer, F. S., Phys. Rev. A, to be published during 1979.

27. GARDNER, L. D., Ph.D. Thesis, Yale Univ., (1978).

28. SIMONEN, T. C., et al., Bull. Am. Phys. Soc. 23775 (1978).

29. SPITZER, L., Physics of Fully Ionized Gases, Ch. 5, 2nd edition, Wiley (1962).

30. FUTCH; A. H., et al., Plasma Physics 14211 (1972).

31. KELLY, R. L., PALUMBO, L. J., Naval Research Laboratory, Report No. 7599. 
32. TERRY, J. L., CHEN, K. I., MOOS, H. W., MARMAR, E. S., Johns Hopkins Univ. Report COO-2711-3.

33. EQUIPE TFR, Nuc. Fus. 151053 (1975).

34. NEXSEN, W. E., TURNER, W. C., CUMMINS, H. F., Lawrence Livermore Laboratory report UCRL-80986 (1978), to be published.

35. NEXSEN, W. E., private communication.

36. BURRELL, C. F., private communication.

37. ULRICKSON, M., private communication.

38. MCWHIRTER, R. W. P., Ch. 5, P. 201, Plasma Diagnostic Techniques, ed. by R. H. Huddlestone and S. L. Lennard, Academic Press (1965).

39. DAVIS, J., Naval Research Laboratory, private conmunication.

40. POST, D. E., JENSEN, R. V., TARTER, C. B., GRASBERGER, W. H., LOKKE, W. A., At. Data and Nuc. Tables 20397 (1977).

41. CARLSON, G. A., et al., Lawrence Livermore Laboratory Report UCRL-52467 (1978).

42. MOIR, R. W., et al., Lawrence Livermore Laboratory Report UCRL-52302 (1977).

43. JASSBY, D. L., Nuc. Fus. 17309 (1977).

44. COENSGEN, F. H., TMX Major Project Proposal, Lawrence Livermore Laboratory, Rept. LLL-Prop-148 (1977).

45. BERKNER, K. H., PYLE, R. V., STEARNS, J. W., Nuc. Fus. 15259 (1974).

46. COENSGEN, F. H., MX Major Project Proposal, Lawrence Livermore Laboratory, Report LLL-Prop-142 (1976).

47. RUDD, M. E., MACEK, J. H., Case Studies In Atomic Physics 3 47 (1972). 48. GRYZINSKI, M., Phys. Rev. 138 A305 (1965). 49. STABleR, R. C. Phys. Rev. 133 Al268 (1964). 50. DRAKE, R. P., CHEN, K. I., MOOS, H. H., TERRY, J. T., HORNADY, R. S., Lawrence Livermore Laboratory report UCID-17864, unpublished, 1978. 
51. STE INhAUS, J. F., OLESON, N. L., BARR, W. L., Phys. Fluids ㅇ, 1720 (1965).

52. CLAUSER, J. F., private communication.

53. TERRY, J. L., Ph.D. Thesis, Johns Hopkins Univ. (1978).

54. ANDERSON, C. A., private communication.

55. ELTON, R. C., Atomic Processes, in Methods of Experimental Physics, 9A, Griem and Luvberg, eds. (1971).

56. WEISE, W., et al., Atomic Transition Pzobabilities, National Bureau of Standards report NSRDS-NBS-4, Vol . 1 (1966).

57. SIMONEN, T. C., et al., Nuc. Fus. 1461 (1974).

58. TURNER, W. C., KAISER, T. B., Bull. Am. Phys. Soc. 23754 (1978) (report in preparation).

59. SIMONEN, T. C., Nuc. Fus. 15542 (1975).

60. OSHER, J. E., Lawrence Livermore Laboratory Report UCRL-81240 (1978). 
Richard Paul Drake was born on October 25, 1954 in Washington, D.C. His early years were spent in Illinois and Colorado. He attended high school and Colorado State University until the fall of 1972, when he entered Vanderbilt University. At Vanderbilt he took a degree in philosophy and physics, benefiting from the instruction and advice of Dr. John P. Barach in the latter field. During his final year he was captain of the fencing team, and he graduated Magna Cum Laude in May of 1975, with Honors in philosophy. From June 1969 through August 1975 he participated in a training program of the Woodward Governor Co., gaining valuable practical experience in the use of machine tools.

Soon after entering The Joins Hopkins University in the fall of 1975, he began working with the plasma spectroscopy group of Dr. H. Warren Moos. Before receiving an M.A. degree in physics in early 1977, he was a Teaching Assistant and studied under Professors C. L. Chen, C. Y. Chen, G. Feldman, and Kovesi-Domokos. He benefited in particular from study under Professor Gabor Domokos. After brief participation in a plasma diagnostics experiment at the Massachusetts Institute of Technology, he conducted a major spectroscopic study of the 2 XIIB mirror machine at the Lawrence Livermore Laboratory during 1977 and 1978. 